\title{
RealTime Implementation Of An Internal-Model-Principle Signal Identifier
}

Edris Saleh Mohsen, The University of Western Ontario

Supervisor: Dr. Lyndon J. Brown, The University of Western Ontario

A thesis submitted in partial fulfillment of the requirements for the Master of Engineering

Science degree in Electrical and Computer Engineering

(c) Edris Saleh Mohsen 2017

Follow this and additional works at: https://ir.lib.uwo.ca/etd

Part of the Controls and Control Theory Commons, and the Signal Processing Commons

\section{Recommended Citation}

Mohsen, Edris Saleh, "RealTime Implementation Of An Internal-Model-Principle Signal Identifier" (2017). Electronic Thesis and Dissertation Repository. 5009.

https://ir.lib.uwo.ca/etd/5009

This Dissertation/Thesis is brought to you for free and open access by Scholarship@Western. It has been accepted for inclusion in Electronic Thesis and Dissertation Repository by an authorized administrator of Scholarship@Western. For more information, please contact wlswadmin@uwo.ca. 


\section{Abstract}

This thesis presents a new means of tuning an adaptive internal model principle based signal identification algorithm whose computational costs are low enough to allow a realtime implementation. The algorithm allows an instantaneous Fourier decomposition of nonstationary signals that have a strongly predictable component. The algorithm is implemented as a feedback loop resulting in a closed loop system with a frequency response of a bandpass filter with notches at the frequencies of the Fourier decomposition. This is achieved through real time selection of the coefficients of the transfer functions in the feedback loop. Previous work showed how the dynamics of the algorithm could be chosen to be represented by a bandpass filter with notches. However this involved solving a large set of coupled linear equations. This thesis shows how the equations can be decoupled into pairs of linear equations which have explicit solutions. In other word, rules for explicitly solving for these parameters are given that only involve evaluating frequency responses at the frequencies of the instantaneous Fourier decomposition. Last but not the least, alternative approach for choosing suitable coefficients to eliminate the DC component of the signal under consideration has been proposed as well by changing frequency response of the closed loop system from bandpass filter to a lowpass filter and adding a model of the constant signal to the feedback loop.

Keywords: Internal model principle, frequency identification, adaptive multiple notch filters, periodic disturbance, instantaneous Fourier decomposition. 


\section{Acknowledgements}

First, I would like to thank my supervisor Dr. Lyndon J. Brown for his support, advise and encouragement during the two years of my study at the University of Western Ontario. I really appreciate everything that he has done for me and it was a pleasure to work with such intelligent and wise professor like him. He was always able to provide me with the knowledge and tips that kept me going forward in my research, also i would to thank him for his patience and respectful behavior.

I would like also to send many thanks to my home country (Libya) and Libyan Ministry of higher education and scientific research for their fund and support during past three years that I spent in Canada despite the hard environment that they work at because of unstable situation in Libya.

Last not the least, I would also like to thank my family and my friends for their support and encouragement during that time. 


\section{Table of Contents}

$\begin{array}{ll}\text { Abstract } & \text { i }\end{array}$

$\begin{array}{ll}\text { Acknowledgements } & \text { ii }\end{array}$

Table of Contents

$\begin{array}{ll}\text { List of Tables } & \text { vi }\end{array}$

List of Figures

List of Abbreviations and Nomenclature $\quad$ xi

1 Introduction 1

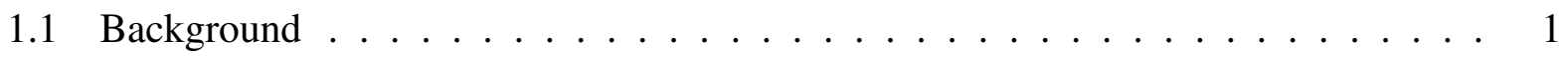

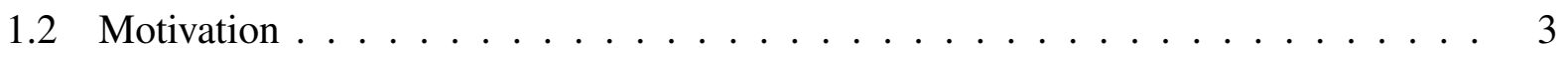

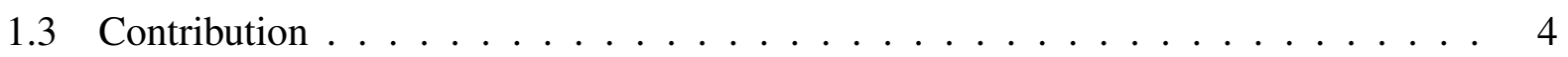

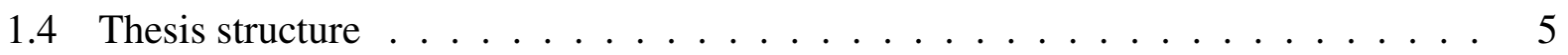

2 Previous Work $\quad 6$

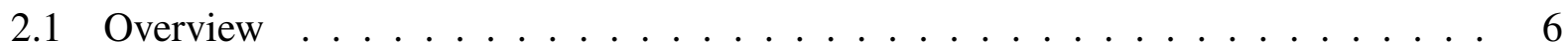

2.2 Control theory literature review . . . . . . . . . . . . . . 6

2.3 Signal processing literature review . . . . . . . . . . . . . . 11

2.3.1 Fast Fourier Transform $(\mathrm{FFT}) \quad \ldots \ldots \ldots \ldots \ldots$

2.3.1.1 Fast filter Bank . . . . . . . . . . . . . . . . . 12 
2.3.2 Wavelet Transform . . . . . . . . . . . . . . . . . . . 12

2.3.3 Hilbert Huang Transform $(\mathrm{HHT}) \ldots \ldots \ldots$

2.3.3.1 Empirical Mode Decomposition (EMD) . . . . . . . . . . . 14

2.3.3.2 Hilbert spectral analysis . . . . . . . . . . . . 15

2.4 Adaptive Notch Filter . . . . . . . . . . . . . . . . . . 16

2.5 Instantaneous Fourier decomposition . . . . . . . . . . . . . . . 17

3 Algorithm Development $\quad 18$

3.1 Review of the (IFD) previous work $\ldots \ldots \ldots \ldots \ldots$

3.1.1 Adaptive algorithm and comparison to HHT . . . . . . . . . . . . 21

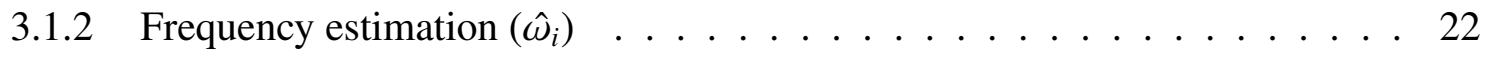

3.2 Control parameter selection $\ldots \ldots \ldots \ldots$

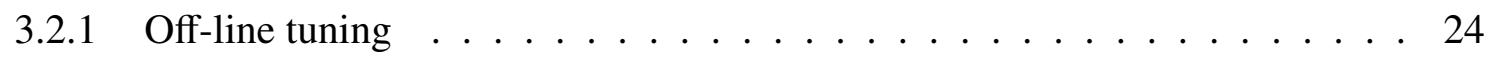

3.2.2 Online controller gain selection $\ldots \ldots \ldots \ldots \ldots$

3.2.2.1 Linear dependency of the equations . . . . . . . . . . 28

3.3 Alternative approach for choosing suitable coefficients of the system controller . . 29

3.3.1 Alternative approach controller parameters calculation $\ldots \ldots \ldots 32$

3.3.2 State Space Form of (IFD) within discrete time $\ldots \ldots \ldots \ldots$

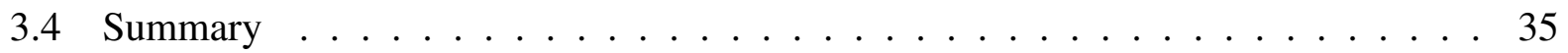

4 Simulation Results and Comparison $\quad 36$

4.1 Simulation and Comparison . . . . . . . . . . . . . . . 36

4.1 .1 Computing a Tuning Function $\ldots \ldots \ldots \ldots$

4.1 .2 Frequency identification $\ldots \ldots \ldots \ldots$. . . . . . . . 40

4.1.3 Harmonics magnitudes of the synthesized signal . . . . . . . . . . . . . 45

4.1 .4 Comparison of true and identified signal . . . . . . . . . . . . . 47

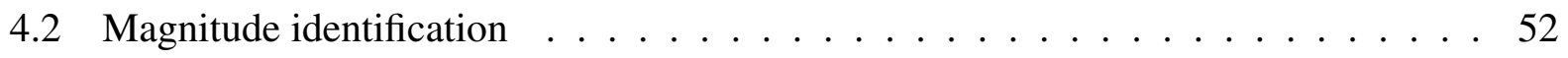

4.2 .1 Comparison with HHT algorithm . . . . . . . . . . . . . 56 
4.3 Summary . . . . . . . . . . . . . . . . . . . . 61

5 Conclusions and Future Directions $\quad 62$

5.1 Conclusion ......................... 63

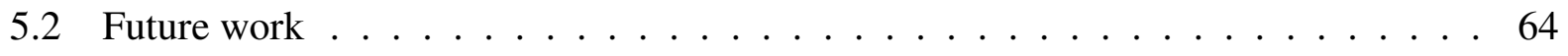

$\begin{array}{lll}\text { Appendix A } & \text { Frequency identification(continue time) } & 71\end{array}$

A.1 Off-Line tuning $\ldots \ldots \ldots \ldots \ldots \ldots \ldots \ldots \ldots \ldots \ldots \ldots \ldots \ldots \ldots$

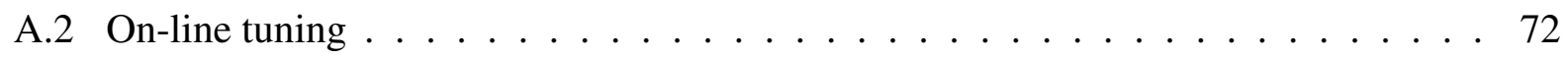

$\begin{array}{lll}\text { Appendix B Discrete Time } & \mathbf{7 6}\end{array}$

$\begin{array}{lll}\text { Appendix C Proposed algorithm Matlab code } & 81\end{array}$

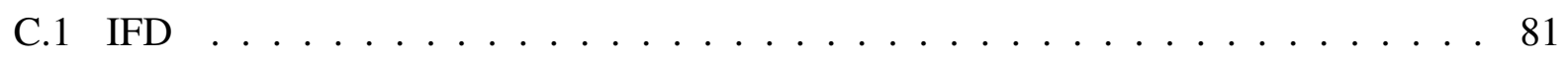

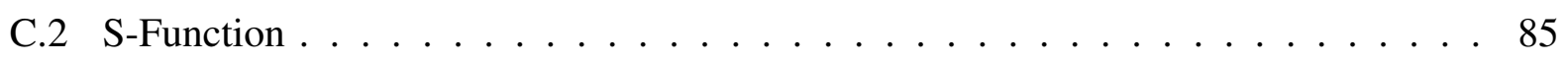

$\begin{array}{lll}\text { Appendix D Mat-lab alternative approach code } & 87\end{array}$

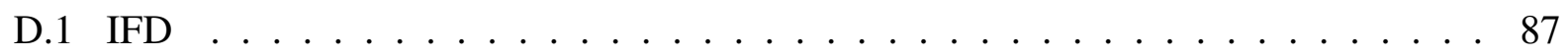

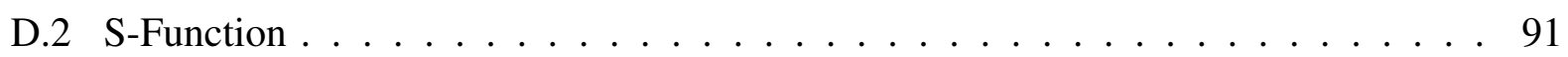

$\begin{array}{lr}\text { Curriculum Vitae } & 93\end{array}$ 


\section{List of Tables}

4.1 Bandpass filter parameters $\ldots \ldots \ldots \ldots$

4.2 Values of simple tuning function $\mathrm{G}(\mathrm{s}) \ldots \ldots \ldots \ldots$

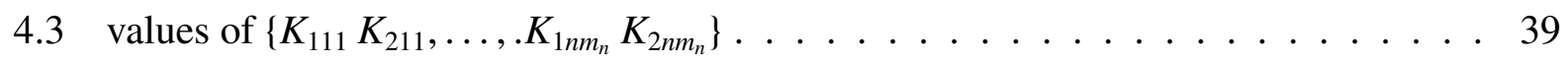

4.4 Simple, proposed and alternative algorithm error comparison . . . . . . . . . 52 


\section{List of Figures}

3.1 Simple tuning instantaneous Fourier decomposition block diagram . . . . . . . . . 19

3.2 Developed instantaneous Fourier decomposition block diagram . . . . . . . . . . 22

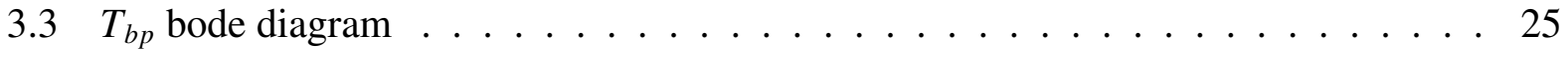

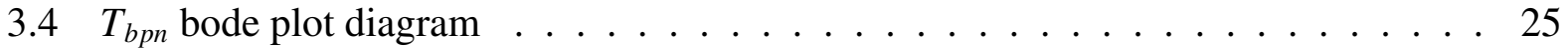

3.5 Structure of alternative proposed instantaneous Fourier decomposition . . . . . . 30

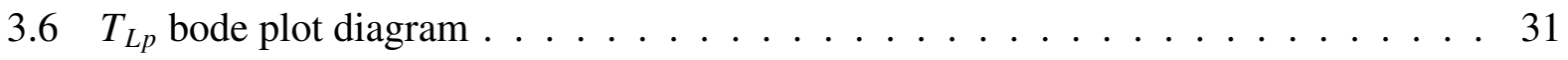

4.1 Block diagram of periodic signals generator . . . . . . . . . . . . 37

4.2 Periodic Disturbance . . . . . . . . . . . . . . . . . . 38

4.3 Frequency identification for first fundamental component of proposed tuning func-

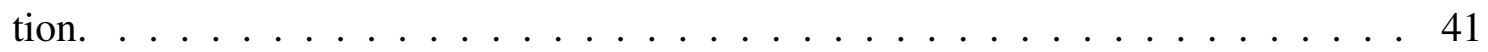

4.4 Frequency identification for second fundamental component of proposed tuning

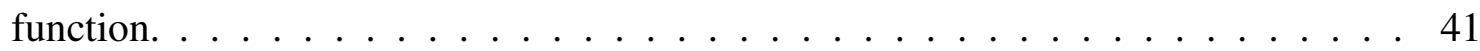

4.5 Frequency identification for first internal model of simple tuning function. . . . . . 42

4.6 Frequency identification for second internal model of simple tuning function . . . . 42

4.7 Fast Fourier transform of the input signal and error $\ldots . . \ldots 43$

4.8 Fast Fourier transform of the input signal and error for simple tuning function . . . 44

4.9 Fast Fourier transform of the input signal and error for alternative approach $\ldots 45$

4.10 Magnitude of each harmonic in the $1^{s t}$ internal model . . . . . . . . . . . . 46

4.11 Magnitude of each harmonic in the $2^{\text {nd }}$ internal model $\ldots . . \ldots 46$

4.12 Identified versus true signal for the first set of the internal model . . . . . . . . . 47

4.13 Identified versus true signal for the second set of the internal model . . . . . . . . 48 
4.14 Comparison of identified signal, error and real signal with proposed tuning function 49

4.15 Comparison of identified signal, error and real signal without a DC component of proposed tuning function $\ldots \ldots \ldots \ldots$. . . . . . . . . . . . . .

4.16 Comparison of identified and true signal of alternative proposed algorithm . . . . 51

4.17 Error signals for proposed tuning versus simple tuning and alternative approach . . 52

4.18 Identified amplitudes of first set of the $\mathrm{d}(\mathrm{t})$ harmonic. . . . . . . . . . . . . 54

4.19 Identified amplitudes of second set of the $\mathrm{d}(\mathrm{t})$ harmonic. . . . . . . . . . . . . 54

4.20 Frequency identification for first fundamental component alternative proposed al-

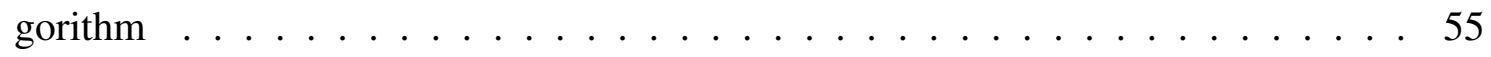

4.21 Frequency identification for second fundamental component alternative proposed

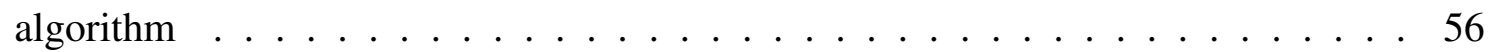

4.22 IMFs for the first four components . . . . . . . . . . . . . . . . . . 57

4.23 IMFs for $5^{t h}, 6^{t h}, 7^{t h}$ and $8^{t h}$ components . . . . . . . . . . . 58

4.24 IMFs for $9^{\text {th }}, 10^{\text {th }}, 11^{\text {th }}$ and $12^{\text {th }}$ components . . . . . . . . . . . . 58

$4.25 \mathrm{IMFs}$ for $13^{\text {th }}, 14^{\text {th }}, 15^{\text {th }}$ and $16^{\text {th }}$ components . . . . . . . . . . . 59

$4.26 \mathrm{IMFs}$ for $17^{\text {th }}, 18^{\text {th }}, 19^{\text {th }}$ and $20^{\text {th }}$ components . . . . . . . . . . . . 59

4.27 IMFs for $21^{s t}, 22^{\text {nd }}, 23^{\text {rd }}$ and $24^{\text {th }}$ components . . . . . . . . . . . . . 60

4.28 IMFs last component $\ldots \ldots \ldots$. . . . . . . . . . . . . . . . . . 60 


\title{
List of Abbreviations, Symbols,
}

\section{Nomenclature}

\author{
HHT Hilbert Huang Transform \\ $j \hat{w}_{i} \quad$ Notches frequencies \\ $\epsilon_{i j} \quad$ Small real number \\ $\hat{\omega}_{i} \quad$ Estimated frequency \\ $\omega_{i} \quad$ Fudamental frequency \\ $\phi_{i j}(t) \quad$ Relative phases \\ $\psi_{\ell}(t) \quad$ Mother wavelet \\ $A_{i j} \quad$ Harmonic amplitudes \\ $d_{i}(t) \quad$ The component of signal to be identified \\ $K_{i} \quad$ Adaptive gain \\ $K_{1 i j}, K_{2 i j}$ Feedback controller gains \\ $K_{p} \quad$ Control gains \\ $m_{i} \quad$ Number of harmonics of the fundamental frequency
}


$T_{b p}(\mathrm{~s}) \quad$ 2nd Order desirable bandpas filter

$T_{L p} \quad$ Transfer function of lowpass filter

$x_{1 i j}, x_{2 i j}$ State spaces variables

ANF Adaptive notch filter

$\mathrm{d}(\mathrm{t}) \quad$ Signal to be identified

DFT Discrete Fourier Transform

DIT Decimation In Time

DWT Discrete Wavelet Transform

EMD Empirical Mode Decomposition

FFC Feed Forward Cancellation

FIR Finite impulse response

G(s) Tuning Function

HSA Huang Spectral Analyzes

i Index counter

IFD Instantaneous Fourier Decomposition

IIR Infinite impulse response

IMF Internal Model Function

IMP Internal Model Principle

IMs Internal Models 


$\begin{array}{ll}\mathrm{j} & \text { Index counter } \\ \mathrm{K} & \text { Integrator } \\ \text { LMS } & \text { least mean squared } \\ \mathrm{n} & \text { Number of fudamental frequency } \\ \mathrm{n}(\mathrm{t}) & \text { Measurment noise } \\ \mathrm{NF} & \text { Notch filter } \\ \text { PI } & \text { A proportional integral } \\ \text { PLL } & \text { Phase Locked Loop } \\ \text { RLS } & \text { recursive least squares } \\ & \\ \text { RSW } & \text { Resistance Spot Welding } \\ \text { WT } & \text { Wavelet Transform }\end{array}$




\section{Chapter 1}

\section{Introduction}

\subsection{Background}

In control theory, communication, signal processing, and mechanical systems, it is common to encounter signals that include components that can be predicted based on their past values but whose characteristics slowly vary with time. These signals can be modeled by the following equation

$$
d(t)=\sum_{i=1}^{n} d_{i}(t)=\sum_{i=1}^{n} \sum_{j=1}^{m_{i}} \bar{A}_{i j}(t) \sin \phi_{i j}(t)+n(t)
$$

where

$$
\phi_{i j}(t)=\int_{0}^{t} j \omega_{i}(t) d t+\phi_{i j}(0)
$$

and $n(t)$ is noise. $\mathrm{d}(\mathrm{t})$ is the sum of $n$ periodic components with each component composed of $m_{i}$ harmonics. The periods, the harmonic amplitudes and relative phases can vary slowly in time. In signal processing, communicatins and other scientific fields there is great value in determining the values $\omega_{i}, \bar{A}_{i j}$ and $\phi_{i j}-\phi_{11}$ in equation (1.1). In this work, $\mathrm{j}$ is used as a counter, never the imaginary number. There are many algorithms that have been proposed to identify these signals including the most traditional technique, the fast Fourier transform and newer techniques 
including wavelet analysis, Gabor analysis [16] , approaches that are based on adaptive notch filters [35] and output regulation [17]. A new approach that has been widely applied is the Hilbert Huang Transform (HHT) [33]. These are discussed in the literature review. A similar problem is encountered in the control theory literature where exact tracking of reference signals or rejection of disturbances is often required. Approaches that accomplish this include repetitive controllers [36] and adaptive feed-forward cancellation (AFC) [4]. A fundamental control theory principle is the internal model principle (IMP). This principle was presented by Francis and Wonham and states that the output error can be driven asymptotically to zero by placing a model of exogenous signals in a stable feedback loop [14]. In this framework, a model of the signal is the Lapalce transform of that signal. Unfortunately small errors in this model can lead to significant degradation in the performance of internal model principle controllers. This problem of uncertainty in the signal model can be overcome with adaptive controllers [6]. In achieving asymptotically perfect rejection of disturbances it is inherent that the disturbance is completely identified. Thus, these type of controllers can be turned into signal processing algorithms by replacing the process to be controlled with tuning functions [41]. This is the approach that will be pursued in this thesis.

Unfortunately, to successfully implement this algorithm requires being able to tune a stable feedback control loop for the entire range of possible frequencies in the model given by equation (1.1). Fortunately, it has been shown that in the signal processing framework, the simplest tuning solution, i.e. selecting all of the gains to be one, is guaranteed to be stable [31]. This algorithm has been successfully applied to the problem of the repeatable disturbances seen in disk drive head control [27]. Unfortunately, by resorting to this simple tuning approach, there is no control over the dynamics and noise rejection characteristics of the algorithm. Instead, this thesis follows the approach introduced in [50] but finds an implementation that is compatible with realtime constraints.

In optical disk drives the laser beam spot must be located exactly on the track-way in order to read the data. However, in the presence of disturbance components an error of tracking appears, which affects the performance of tracking. Therefore, it is important to eliminate this periodic 
disturbance in order to reduce the error to lower level and achieve high stability performance of the control system, so a repetitive controller is applied to the designed controller to improve the performance of tracking, and maintain the stability of the system with unknown process coefficients [21].

In our research we studied one of these adaptive algorithms, which is so-called an adaptive internal model principle algorithm that was developed by L. J. Brown and Q. Zhang for identification of periodic signals with uncertain frequencies in 2003 and 2006 [7] [51]. Then, as modified by Y. Sun in 2006 [40] by using instantaneous Fourier decomposition (IFD) algorithm that based on the (IMP), and recently was extended by Y. Ma in 2009 [28]. When the frequencies are known a prior, the report [50] shows how the dynamics of the algorithm can be completely specified. Unfortunately this article requires setting up and solving a set of more than $2 n_{i}=2 \sum m_{i}$ coupled linear equations which are a function of the signal's frequencies. Unless the sample rate is less than $1 \mathrm{~Hz}$ this will not be feasible to do each sample. This thesis shows how these parameters can be explicitly solved by simply evaluating some frequency response functions at certain frequencies.

\subsection{Motivation}

It is important when you modify or develop an algorithm to take into account the following question: Are the computational costs of the algorithm low enough to allow a realtime implementation? Answering this question was our main motivation in developing this algorithm, since the algorithm has been successfully implemented offline on many applications with known frequencies such as power system [53], musical pitch tracking [52], audio signal decomposition [28], and dynamic resistance measurement in spot welding [40]. This algorithm has also shown powerful frequency estimation and disturbance rejection on applications with unknown but few frequencies or with minor variations in the frequencies. Instantaneous frequency estimation that is used to decompose the signal into sum of narrow band signals, first was applied to resistance spot welding (RSW) by Malhotra [29]. The algorithm presented in [29] has shown good signal decomposition performance 
with narrow band signals and known frequencies. However, it has poor transient response with the infinite number of harmonics that weld data is composed of. The adaptive algorithm has shown high performance in frequency identification and noise cancellation, it has some major restrictions that limit this method. First, how many components that a periodic signal has should be known in advance. Removing this restriction will be left to future work. In addition, previous to this work, it was necessary to be able to design appropriate feedback controller for each frequency that guarantee the system stability, which is considered as a difficult and main limitation. Moreover, the convergence speed of the estimate frequency is quite slow with high level over shoot due to the selection of the adaptive gain. Solutions are proposed to solve these limitations by developing tuning function $\mathrm{G}(\mathrm{s})$ and internal model transfer function $I M_{i, j}$. In prior work, when the frequencies are known, a second order Chebyshev band-pass filter with several notches is designed $\left(T_{b p n}\right)$, so appropriate parameters can be calculated $K_{1 i j}, K_{2 i j}$ for the transfer function of the feedback controller. In this work, we show how these gains can be calculated in realtime with sample rates up to $4 \mathrm{kHz}$. As a result, system stability can be guaranteed, high tracking performance and a periodic signal identification with uncertain frequency are achieved. Furthermore, updating estimate frequency $\hat{\omega}_{i}$ by employing adaptive gain $K_{a}$ faster convergence is achieved with acceptable over shoot level.

Consequently, in this thesis, system stability and high tracking accuracy are achieved and in real time, which have never done before.

\subsection{Contribution}

In this thesis the main contributions are assigned as follows:

1. The main contribution in our research is first to implement the algorithm in realtime that was proposed by Y. Ma in 2009.

2. Another major contribution was dealing with the issue of the set of equations becoming linearly dependent. Whenever the frequencies are equal to $0.01 \%$, numerical stability issues 
cause the algorithm to become unstable.

3. We also modified the previous method that was based on a bandpass filter with a new approach based on a low pass filter and explicit identification of the constant component of the signal.

The simulation results demonstrate the validity of our algorithm and the ability to identify the frequency with uncertain periodic, and reject the sinusoidal disturbance in real time, which has never been obtained before.

\subsection{Thesis structure}

- In chapter 2, previous work in this area is presented and reviewed as a literature review.

- In chapter 3, our a new development on the IFD algorithm that is based on the orthogonal state variables of an internal model principle controller and alternative proposed approach are indicated and well explained, also it shows how the equations can be decoupled into pairs of linear equations. Moreover, it is compared with the previous work that had been done by L. J. Brown and his team research.

- In chapter 4, we show how the instantaneous Fourier decomposition is implemented in real time, and the frequency is identified with high speed of convergence as well as the system stability of the modified algorithm. In addition, to the comparison results with other algorithms.

- In chapter 5, our work in this thesis is concluded, and the recommended work for the future is outlined. 


\section{Chapter 2}

\section{Previous Work}

\subsection{Overview}

In this chapter a literature review for previous work related to our research area is presented for both basic control and signal processing algorithms. Furthermore, a brief introduction of our adaptive algorithm is given at end of this chapter and explained in detail in the next chapters.

\subsection{Control theory literature review}

Several approaches are proposed for system variables control and disturbance cancellation with uncertain behaviors of the periodic signal. The most common algorithm is the internal model Principle of control (IMP) presented by B. A. Francis and W. M. Wonham in 1976 [14]. The objective of an internal model is to reject disturbances or provide set-point signal by providing close loop transmission zeros that eliminate the critically stable poles of the periodic disturbance or set point. However, this principle requires prior information of the frequency of the signal. In other words, for higher performance, the controller must have pair of poles on the $j \omega$ axis that equal the disturbance frequency, and the fact that many problems have an uncertain frequency is considered is the main restriction of this approach.

In 1988 an extension schema called the repetitive control system that is based on the internal 
model principle was presented by S. Hara, Y. Yamamoto and T. Omata and M. Nakano [36]. It shown the ability of controlled variables to track a periodic disturbance that lead to decrease the tracking error to the low level and reject the disturbances. However, there is a trade-off between the stability and tracking accuracy performance of the system because of the positive feedback loop that restricted the controller gains due to the phase lag when there is frequencies crossing over. Therefore, it is difficult to design a repetitive control with unknown plant parameters. In [9] four discrete time repetitive controls were compared. Since previous algorithms are based on the internal model principle, they need prior information of the period of the signal. Meanwhile, other algorithms were presented to restrict this requirement. For instance, in 1998 repetitive control for the tracking references of servo systems such as an optical disk drive was developed by J. H. Moon, M. N. Lee and M. J. Chung [21]. The repetitive controller was designed based on graphical technique of the Nyquist envelope that relies on the frequency domain. Therefore, the stability of the system is enhanced as well as high performance of tracking accuracy is maintained. However, the feedback $\mathrm{C}(\mathrm{s})$ was designed independently of repetitive controller which is well designed to guarantee high tracking performance with good system stability assuming that the low-pass q(s) filter is well chosen to keep Nyquiest plot out side the circle of $|q(j \omega)|$ which is difficult to design $q(s)$ with uncertain process that make studying the behavior of of the system so complicated.

Another different technique that is based on the phase-locked loop was proposed by M. Bodson, A. Sacks and P. Khosla in 1992 which is so-called adaptive feed-forward cancellation (AFC) [4]. Although the higher order harmonic is attenuated with good system stability, this schema shown that in order to cancel the periodic disturbance, the gains of the process must be calculated for all estimated frequencies which is not reliable within a complicated plant. In addition, there is a limitation of rejection on a certain number of frequency components.

In 1997 two algorithms were given by M. Bodson and S. C. Douglas for the cancellation of periodic disturbances with unknown frequency. First, a direct algorithm was presented where the frequency and magnitude were updated by using a single error signal simultaneously. It is based on the phase-locked loop technique exactly similar to (AFC). However, in order to estimate the 
CHAPTER 2. PREVIOUS WORK

frequency, the gain of the plant transfer function at the nominal frequency is required which is not practicable with a complicated processes [49]. Second, an in-direct algorithm which is a combination of (AFC) with an estimated frequency algorithm. In this schema, the frequency is estimated separately from the rejection algorithm to provide what so-called indirect algorithm. This approach has the ability to reduce the sinusoidal disturbances with uncertain frequency ;however, it has low performance accuracy convergence because of the signal used to estimate a frequency disturbance which is eliminated by the control input $[26,25,3]$.

High performance tracking of a periodic signal and disturbance cancellation with uncertain frequency is a crucial and significant topic, especially in control theory. Therefore, several adaptive algorithms were previously developed. L. J. Brown and Q. Zhang developed a new algorithm for canceling periodic disturbances and identification of periodic signals with uncertain frequencies in 2001-2003 [6, 7, 50,51]. In [6] the approach is based on the state space variables of an internal model principle of control. Also traditional feedback controller ( PI) is used in parallel with the internal model principle. It shown high performance to reject a periodic disturbance and identify the frequency sinusoidal disturbance. However, the gain of the controller is limited when there is cross over frequency. Therefore, the system stability is not guaranteed in all times. Also the implementation was done in absence of input noise, which may not give same results in presence of noise [6]. In [7] the algorithm was applied to identify a periodic signal. However, one of the main limitations in this algorithm is when the fundamental frequency of the periodic disturbance is too low, then the frequency cannot be identified.

Noise analysis of the algorithm for uncertain frequency identification was presented by L. J. Brown and Q. Zhang in 2006 as an extension of those described earlier which used band-pass filter with notches as the controller [51]

Rejection of unknown periodic disturbance in magnetic hard disk drives was proposed in 2007 by M. Nagashima, K. Usui and M. Kobayashi. It is completely based on modified internal model principle presented by L. J. Brown and Q. Zhang; however, the main limitation of this approach is if the control system has more than one input (more than one signal component), and in order to 
eliminate all disturbances more controllers must be placed in the system otherwise the controller not only cannot cancel the unaddressed frequency but also the disturbance frequency that would be addressed. That leads to more complicated system with high cost, which is also incapable to work in real time [27]. Similarly in $2008 \mathrm{H}$. Li, C. Du and Y. Wang proposed approach to cancel a disturbance in hard disk drivers HDDs. In this method the signal was rebuilt, so the disturbance can be approximated and canceled by adding an opposite value of this rebuilding signal. Their approach was based on an adaptive internal model based principle for frequency identification and disturbance cancellation proposed by L. J. Brown and Q. Zhang in [8]. Since in this method the disturbance re-builder should add after the estimation frequency convergence, this cause a rebuilding delay, which is considered as main limitation. In deed, the implementation was done in absence of the noise [18].

Extending to the previous work adaptive rejection of stochastic and deterministic periodic disturbance was proposed by Y. Wang, K. C. Chu and T. C. Tsao in 2009. It is based on the adaptive internal model with adaptive notch filter, so the frequency estimation is achieved and the system can provide perfect parameters that proof noise cancellation. However, using two adaptive channels for uncertain deterministic periodic cancellation and norm minimization (output variance minimization) has adverse effects such as they can interfere and prevent the convergence. Therefore, the system cannot be guaranteed to be stable for all times. In addition, there is a trade-off between the convergence speed and requirements of computational resources by using QR-RLS algorithm [34, 47].

Further, in 2009 L. J. Brown and Y. Ma applied modified adaptive instantaneous Fourier decomposition technique that based on the internal model principle (IMP) on the music signal. The signal was analyzed and decomposed into narrow band in shape of time frequency. It is shown high performance tracking signal accuracy with high system stability. However, this approach was restricted by specific type violin music signal. In other words, it may not applicable for other instruments signal, especially those who have high noise level. Therefore, the algorithm has to modified [28]. 


\section{CHAPTER 2. PREVIOUS WORK}

Recently, several approaches were proposed in this particular area either based on the phaselocked loop such as On-line adaptive identification and cancellation of periodic disturbance with unknown parameter that was proposed by Y. Xu, D. Huang and L. Zhang in 2009 [48], or based on the adaptive internal model principle such as disturbance cancellation for linear systems by adaptive internal models that was proposed in 2013 by R. Marino and P. Tomei [30]. 
CHAPTER 2. PREVIOUS WORK

\subsection{Signal processing literature review}

A variety of transformation approaches and/or techniques that used to analysis the signal and identify the frequency. One of the most common schema is to decompose the signal into sum of narrow band components each correspond one harmonic generating Fourier representation of the input signal. Time frequency representation is the base of the multiple fundamental frequency identification [28]. A several papers and books are published in this particular area, so details can be found in $[2,5,24,39,38]$

\subsubsection{Fast Fourier Transform (FFT)}

Fourier transform is one of the most efficient signal analysis algorithms that is used in signal processing and other varying applications. There are several algorithms of Fourier transform used to represent data in form of frequency domain. Earlier computing discrete Fourier transform (DFT) is based on the $O\left(N^{2}\right)$ process that increases the complexity of the discrete Fourier transform computation especially when the data sets have thousands or even millions of channel $\mathrm{N}$ numbers. Using Fast Fourier transform (FFT), the DFT can be computed in $O\left(\operatorname{Nlog}_{2} N\right)$. As a result the complexity of the computation is reduced. One of the most widely used Fast Fourier transform algorithms is Cooley-Tukey algorithm. A common form of Cooley-Tukey algorithm is radix-2. By applying a radix-2 decimation-in-time (DIT) the DFT size is divided by 2, (N/2) for each recursive step [44]. The DFT is described as following:

$$
X_{k}=\sum_{i=0}^{N-1} x(i) e^{(-2 \pi \sqrt{-1} k i / N)}
$$

where $0 \leq k \leq(N-1)$ is the frequency index bin, $x(i)$ represent data in time domain, and $X_{k}$ represent the data in frequency domain. From equation (2.1) it is obvious that DFT is restricted to a fixed set of frequencies. Fourier transform is not based on an adaptive basis, the frequency is derived by convolution. In other words, Fast Fourier transform is applicable only for linear and stationary data analysis. Moreover, the estimation of the frequency of this algorithm is sensitive to 
noise [13].

\subsubsection{Fast filter Bank}

Although the DFT transform can be applied to any complex data. However, the weakness of the band-pass response and the frequency selectivity are considered to be drawbacks of the FFT as a filter bank. Therefore, the fast filter bank designed by Y. C. Lim and B. F. Boroujeny tried to overcome these issues. In order to implement the filter bank with better frequency selectivity and flat band-pass response, increasing the order of the filters is required [23]. This improves the

performance of the frequency response, and low complexity is achieved. Applications with high frequencies are limited by a higher complexity of the computation as the frequency's harmonic of the components to be estimated is higher than $50 \mathrm{~Hz}$ [1]. Therefore, a fast filter bank for an adaptive Fourier analysis and node modulated fast filter were proposed in 1998 and 2003 respectively [1] [42]. Although these algorithms have a lower complexity, they are still difficult to implement because of the their complex varying structures [42].

\subsubsection{Wavelet Transform}

The wavelet transform is a mathematical technique that is used to decompose the signal into sum of local linear, time-dilated and time-translated wavelet components. A wavelet a small picture of the wave, which has oscillation and amplitude; however, the different from the Fourier analysis the wavelet has finite energy focused in the a narrow window. In other word, the wavelet states from zero then increases before goes down to zero again[11, 12, 29].

so let us assume that our function $f(t)$ is represented as a linear decomposition by

$$
f(t)=\sum_{\ell} a_{\ell} \psi_{\ell}(t)
$$

where $\ell$ is an integer index for finite or infinite sum, $a_{\ell}$ is the real value expansion coefficient, and $\psi_{\ell}(t)$ is an expansion set. So, if the basis is orthogonal we obtain 


$$
\left\langle\psi_{k}(t), \psi_{\ell}(t)\right\rangle=\int \psi_{k}(t) \psi_{\ell}(t) d t=0 \quad k \neq \ell
$$

and the coefficients are calculated by the inner product as,

$$
a_{k}=\left\langle f(t), \psi_{k}(t)\right\rangle=\int f(t) \psi_{k}(t) d t
$$

according to [5] and by substituting (2.2) in (2.4) and using (2.3) provide the single $a_{k}$ coefficient. Thus, equation (2.2), can be rewritten as

$$
f(t)=\sum_{k} \sum_{j} a_{j, k}, \psi_{j, k}(t)
$$

where $j$ and $k$ are integer indices, and $\psi_{j, k}(t)$ is the wavelet expansion function.

The set of expansion parameters $a_{j, k}$ are called the discrete wavelet transform (DWT) of the signal $f(t)$, and the equation (2.5) is the inverse transform. The integral wavelet transform is defined as

$$
X(a, b)=\frac{1}{\sqrt{a}} \int_{-\infty}^{\infty} \Psi \overline{\left(\frac{t-b}{a}\right)} x(t) d t
$$

where $\mathrm{a}$ is the scaling factor and $\mathrm{b}$ is the translation. This algorithm is well proved for approximation data with sharp discontinuities. However, one of the main shortcoming is difficulty of design the right mother wavelet for a given application. More details are given in $[5,11]$.

\subsubsection{Hilbert Huang Transform (HHT)}

Most established signal analysis approaches are based either on linear and stationary assumptions such as a Fourier transform, or nonlinear and stationary assumptions such as a wavelet transform. Most of the data either natural or man made are nonlinear, non-stationary and stochastic processes. Therefore, the Hilbert Huang Transform method based on the empirical mode decomposition (EMD) has recently been developed by Huang (1996) [20] to meet this requirement.

Hilbert Huang Transform is an approach that has two main parts: first is the empirical mode decomposition (EMD). Second is the Huang spectral analysis (HSA) . 


\subsubsection{Empirical Mode Decomposition (EMD)}

In this part the signal is decomposed into different components. Therefore, a complicated signal is able to be broken down into finite components. Each intrinsic mode corresponds to simple oscillations; so the intrinsic mode function (IMF) can have a varying magnitude and frequency as a function of time, instead of a fixed magnitude and frequency [20].Then the function can be decomposed as follows:-identify the local extrema, then link the entire local extrema to produce the upper envelope. Repeat the procedure for local minima to obtain the lower envelope. This procedure is called a sifting process $[33,45]$.

$$
h_{1}=X(t)-m_{1}
$$

where $m_{1}$ is the mean of the envelopes, $X(t)$ the data, and $h_{1}$ is the first component (difference between the data and it's mean). Now after the first round of sifting process a new upper and lower envelope of the function are created. Since decomposing signal into (IMF) is required, the sifting process needs to be repeated. In breaking down the signal process, the highest frequency is obtained in the first IMF and a lower frequency is obtained in each subsequent IMF

$$
h_{1}-m_{11}=h_{11}
$$

in the next step $h_{1}$ is treated as data. After repeat the process $k$ times, the $h_{1 k}$ come to be intrinsic mode functions (IMF).

$$
h_{1(k-1)}-m_{1 k}=h_{1 k}
$$

therefore, $h_{1 k}$ selected as a first IMF component

$$
c_{1}=h_{1 k}
$$




\subsubsection{Hilbert spectral analysis}

Once IMF components are obtained, it is easy to apply Hilbert transform to each IMF, then the instantaneous frequency is simply defined in (2.14). More relevant information is pointed out in $[33,20]$. For the original data signal $x(t)$, its Hilbert transform can be represented as $y(t)$ as follows

$$
y(t)=\mathcal{H}\{x(t)\}=\frac{1}{\pi} \int_{-\infty}^{\infty} \frac{x(\tau)}{t-\tau} d \tau=x(t) * \frac{1}{\pi t}
$$

with analytic complex signal is defined as

$$
z(t)=x(t)+i y(t)=a(t) e^{i \Theta(t)}
$$

and

$$
a(t)=\sqrt{x^{2}(t)+y^{2}(t),} \quad \theta(t)=\arctan \left(\frac{y(t)}{x(t)}\right)
$$

where $a(t)$ and $\theta(t)$ in (2.13) instantaneous amplitude and phase respectively, and $\omega(t)$ the instantaneous frequency of $x(t)$ can be expressed as

$$
\omega(t)=\frac{d \theta(t)}{d t}
$$

After applying Hilbert transform to each (IMF) components, then the original data is represented as real part of the following (2.15)

$$
x(t)=\mathfrak{R} \sum_{j=1}^{n} a_{j}(t) e^{i \int \omega_{j}(t) d t}
$$

because of the variability of both amplitude $a_{j}$ and frequency $\omega_{j}$ in (2.15) compared with Fourier transform in (2.1) Hilbert Huang Transform HHT has the capability for analyzing nonlinear non-stationary data. However, main limitations of HHT method during sifting procedure is the empirical mode decomposition can not separate components when their frequencies are adjacent, which is known as mode mixing $[10,33]$. The end effect of the signal is due to the lack of data before and after the data window. Since the signal is limited to be narrow band, the Hilbert 
transform in (2.11) can be approximated as follows

$$
y(t)=\mathcal{H}\{x(t)\} \approx-\frac{1}{\omega} \dot{x}(t)
$$

\subsection{Adaptive Notch Filter}

Adaptive notch filter (ANF) is one of the most important technique that has several applications in noise cancellation, signal processing, echo cancellation, adaptive feedback cancellation and direction-of-arrival of narrow-band signals in radar arrays [35, 37]. (ANF) have become commonly used in some devices that we use in our daily bases such as cell phones and hearing ads. In several papers and books the second order of infinite impulse response (IIR) form of the adaptive notch filter is considered as following

$$
G\left(z^{-1}, \Omega, \rho\right)=\frac{1-z \cos (\Omega) z^{-1}+z^{-2}}{1-2 \rho \cos (\Omega) z^{-1}+\rho^{2} z^{-2}}
$$

where the parameter $\rho(0 \leq \rho<1)$ is known as the poles contraction factor and $\Omega$ is uncertain notch frequency. Equivalent to (2.17) the analog band stop (notch) filter can be expressed as

$$
H(s)=\frac{s^{2}+\omega_{n}^{2}}{s^{2}+2 \epsilon_{n} \omega_{n} s+\omega_{n}^{2}}
$$

where $s$ is Laplace operator $\omega_{n}$ is the natural frequency and $\epsilon_{n}\left(0<\epsilon_{n} \ll 1\right)$ is the damping ratio. There exist many adaptive notch algorithm. For example, stochastic gradient approach such as least mean squared error (LMS) and normalized least mean square (NLMS), or least square estimation such as recursive least squares filter (RLS). Every algorithm has its advantages and drawbacks which make each one unique from the others. Although LMS is communally used as adaptive approach, its main limitation of the eigenvalue spread of the input correlation matrix compared with RLS. See the $[19,43]$ for more details about these adaptive algorithms. 


\subsection{Instantaneous Fourier decomposition}

An adaptive internal model based principle for frequency identification and disturbance cancellation is first developed and implemented for off-line estimation by L. J. Brown and Q. Zhang in 2001. In [7, 49] the desired system behaves as band-pass filter with single notch. However, the control gains can be selected that stabilize the system in the adaptive state.

Instantaneous Fourier decomposition (IFD) algorithm is adopted from the basic internal model principle. This method was first presented by N. Malhotra in 2005 [29] then developed by Y. Sun in 2006. In [40] the algorithm is developed and implemented in continues time and for offline estimation. The Fourier decomposition system behaves as a band-pass filters with multiple notches. However, the control gains for each internal model (IM) are selected to be fixed to guarantee the system stability (non adaptive). In 2009 Y. Ma extended the previous work to meet the requirement not only for continues time but also for a discrete time that first was proposed Z. Zhao and L. J. Brown in 2004 [52]. The signal is decomposed into narrow band signals based on the orthogonal state variables combined with internal models $\left(I M_{i, j}\right)$. Since multiple notch filters are incorporated in the design, the desired system behave as a band-pass filter with multiple notches. The main restriction of Y. Ma work, the method was modified and implemented on a specific type of the music signal (solo violin music). In other words, the method is not guaranteed to work for any arbitrary signal, especially in presence of heavy noise [28]. Our work is extended to what Y. Ma did in [28]. Thus, the internal model transfer function $\left(I M_{i, j}\right)$ was redeveloped, with the controller gains moved to the output vector resulting in a bumpless transfer nature to the time variations in the controller gains/ $\left\{K_{111} K_{211}, \ldots, . K_{1 n m_{n}} K_{2 n m_{n}}\right\}$ are calculated at each time sample instead of much less frequently in [28]. As a result adaptive instantaneous Fourier transform (IFD) algorithm is extended, and for first time is implemented in real time. 


\section{Chapter 3}

\section{Algorithm Development}

\subsection{Review of the (IFD) previous work}

The main key of our research is the IFD algorithm. Since this approach was first proposed by N. Malhotra in 2005 [29], then developed by Y. Sun in 2006 [40] and recently extended by Y. Ma in 2009 [28]. A brief review is given here relevant to the previous work. Adaptive instantaneous Fourier decomposition adopted from the basic IMP. It's main purpose is to identify or cancel a sinusoidal disturbances. After decomposing the signal into narrow-band signals, uncertain varying frequency can be analyzed and identified as pointed out in $[6,7,28,49,50,51]$. Thus, the instantaneous frequency can be estimated based on the orthogonal state variables associated with each internal model (IM). Similarity, HHT is an approach that is based on EMD, where the EMD is a technique that used to minimize any given data into group of intrinsic mode functions IMF, then the Hilbert transform is applied to get instantaneous frequency data. However, one of the Hilbert Hung transform limitations is the difficulty that EMD has to distinguish between the components that have adjacent frequency time varying $[28,33,45]$.

The structure of the simply tuned instantaneous Fourier decomposition algorithm is shown in Fig 3.1 where $G(s)=1$. The periods, the harmonic amplitudes and relative phases can vary slowly in time, i. e. $\bar{A}_{i j}, \omega_{i}$ and $\varphi_{i j}$ are uncertain magnitude, frequency, and relative phases respectively. 


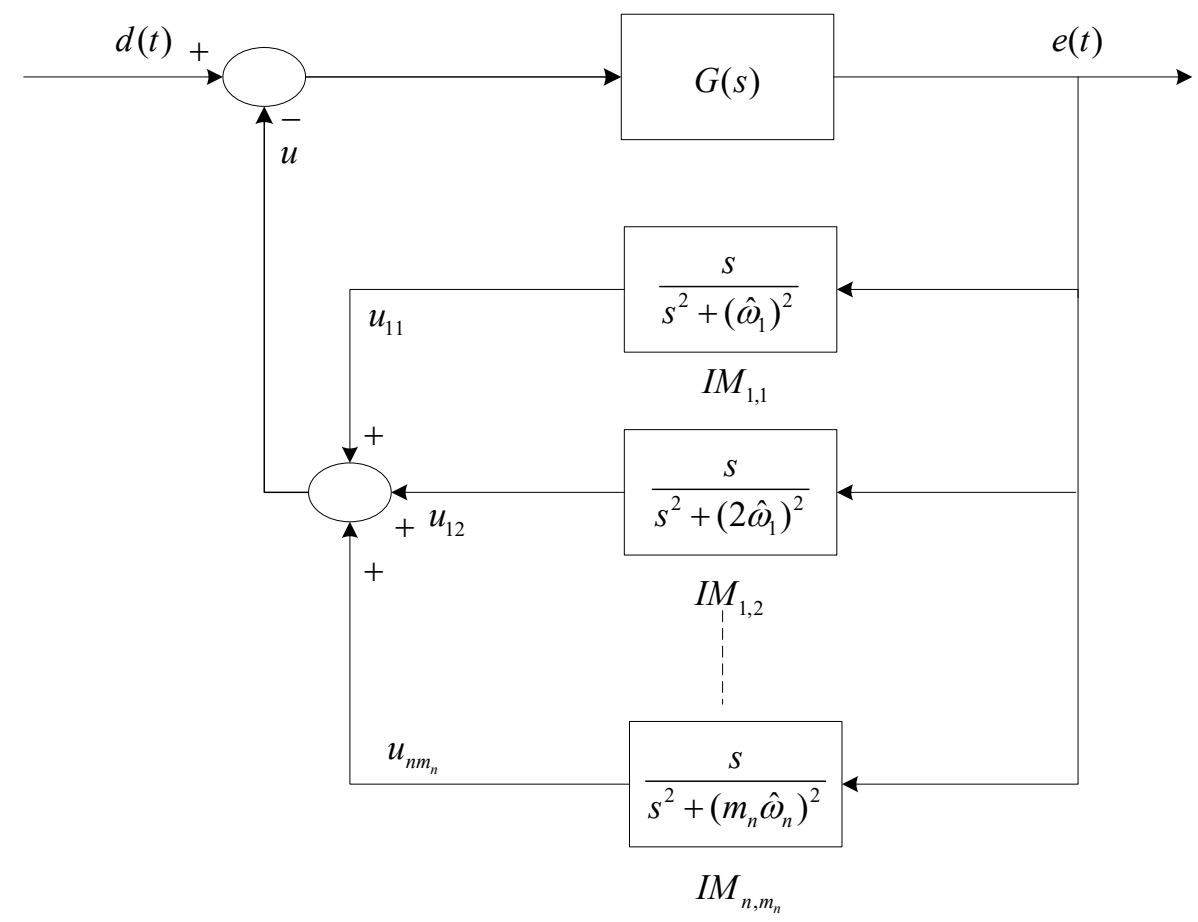

Figure 3.1: Simple tuning instantaneous Fourier decomposition block diagram

This particular structure has the benefit that the closed lop system is stable for all chooses of $n, m_{n}$ and $\omega_{i}$; however, the speed of the closed loop dynamics can vary greatly with the $\omega_{i}$. Each of the transfer functions $I M_{i, j}$ are an internal model for a sinusoidal of frequency $j * \hat{\omega}_{i}$. When the model frequencies and the signal frequencies match, i.e. $j \hat{\omega}_{i}=\omega_{i}$ and the closed loop system is stable, each $u_{i j}$ will be a single sinusoidal and meet the HHT definition of an intrinsic function.

The state space equation for internal model $I M_{i, j}$ is given as

$$
\begin{gathered}
\dot{X}_{i j}=\left[\begin{array}{cc}
0 & j \hat{\omega}_{i} \\
-j \hat{\omega}_{i} & 0
\end{array}\right] X_{i j}+\left[\begin{array}{l}
0 \\
1
\end{array}\right] e \\
u_{i j}=\left[K_{1 i j} K_{2 i j}\right] X_{i j}
\end{gathered}
$$

where,

$$
X_{i j}=\left[\begin{array}{ll}
x_{1 i j} & x_{2 i j}
\end{array}\right]^{T}
$$


and

$$
j \neq \sqrt{-1}
$$

Consequently, the transfer functions from error $e(s)$ to the state variable $x_{2 i j}(s)$ is $s /\left(s^{2}+\left(j \hat{\omega}_{i}\right)^{2}\right)$ and the responses at $x_{1 i j}(t)$ and $x_{2 i j}(t)$ in steady state are:

$$
\begin{aligned}
& x_{1 i j}(t)=\overline{A_{i j}} \sin \left(j \omega_{i} t+\phi_{i j}\right) \\
& x_{2 i j}(t)=\bar{A} \cos \left(j \omega_{i} t+\phi_{i j}\right)
\end{aligned}
$$

and

$$
\begin{aligned}
x_{i j} & =\sqrt{K_{1 i j}^{2} x_{1 i j}^{2}(t)+K_{2 i j}^{2} x_{2 i j}^{2}(t)} \\
\phi_{i j} & =\arctan \left(\frac{x_{2 i 1}(t)}{x_{1 i 1}(t)}\right)-j \omega_{i}(t)
\end{aligned}
$$

thus, the difference $\Delta \omega_{i}$ between fundamental $\omega_{i}$ and estimated $j \hat{\omega}_{i}$ frequency can be presented as

$$
\Delta \omega=\omega_{i}-\hat{\omega}_{i} \approx \frac{e x_{1 i 1}}{x_{1 i 1}^{2}+x_{2 i 1}^{2}}
$$

A applying simple integrator $K_{a}$ to increase the speed of convergence is given as

$$
\frac{d \omega}{d t}=K_{a} \Delta \omega \approx-K_{a} \frac{e x_{1 i 1}}{x_{1 i 1}^{2}+x_{2 i 1}^{2}}
$$

Unfortunately, this leaves the dynamics of the closed loop system uncontrollable and uncertain increase amplification of measurement noise. Therefore, our goals in this thesis not only to modify internal model transfer function to get rid of these limitations but also to meet the realtime requirement by providing less computational algorithm. 


\subsubsection{Adaptive algorithm and comparison to HHT}

The HHT proceeds from the realization that the Hilbert transform gives a mathematically precise definition of instantaneous frequency that agrees with our intuitive understanding when applied to narrowband signals. In this narrowband case, the instantaneous frequency can be approximated as the derivative of the angle of a narrowband signal and $\sqrt{-1}$ times the quadrature of that signal where the quadrature can be approximated by either a scaled version of the derivative or integral of the signal. The HHT uses an empirical method to break down signals into narrowband signals. This empirical method is numerically intensive and not compatible with a realtime implementation [22].

Our algorithm uses the same approximations to estimate the instantaneous frequencies as the HHT but uses an alternative, notch filter based approach that simultaneously calculates the quadrature signals and decomposes the signal into narrow band signals. The structure of the adaptive instantaneous frequency decomposition is shown in Fig. 3.2, where $G(s)$ is a proposed tuning function [31].

Further, with the state space based implementation of the internal models in (3.1) can be rewritten and given by

$$
\begin{aligned}
& \dot{X}=\left[\begin{array}{lll}
A_{11} & & \\
& A_{12} & \\
& & A_{n n_{n}}
\end{array}\right] X+\left[\begin{array}{c}
B_{11} \\
\\
\end{array}\right. \\
& {\left[\begin{array}{c}
u_{11} \\
u_{12} \\
u_{n_{1} n_{n}}
\end{array}\right]=\left[\begin{array}{cccccc}
0 & 1 & \cdot & \cdot & \cdot & 0 \\
0 & 0 & 0 & 1 & \cdot & 0 \\
& & & & & \\
0 & \cdot & \cdot & \cdot & \cdot & 1
\end{array}\right] X}
\end{aligned}
$$

where $X=\left[x_{1 i j} x_{2 i j}\right]^{T}, i=1,2, \ldots, n$, and $j=1,2, \ldots, m_{i} . A_{i j}$, and $B_{i j}$ are expressed as follows

$$
A_{i j}=\left[\begin{array}{cc}
0 & -j \hat{\omega}_{i} \\
j \hat{\omega}_{i} & 0
\end{array}\right] \quad B_{i j=}=\left[\begin{array}{l}
K_{1 i j} \\
K_{2 i j}
\end{array}\right]
$$




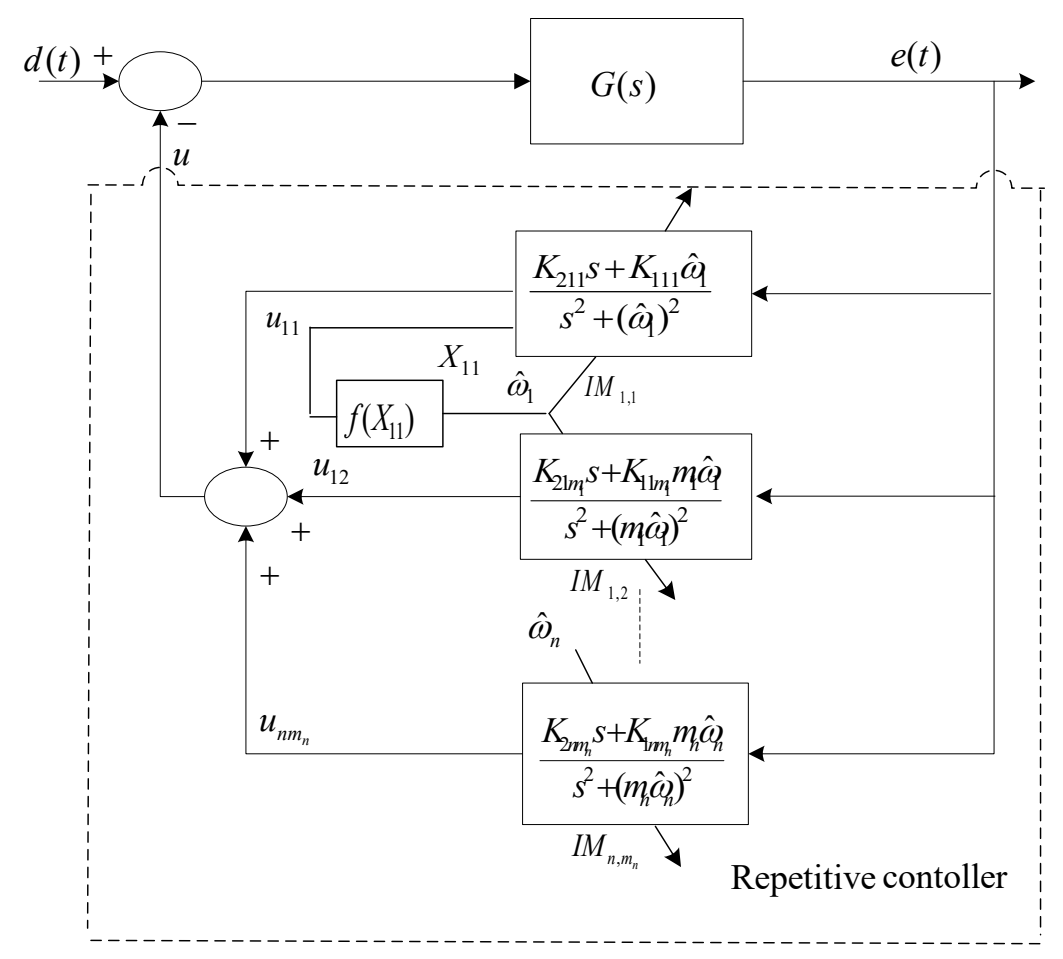

Figure 3.2: Developed instantaneous Fourier decomposition block diagram

This is taken from [7] with minor modifications to fit the signal model that was given in equations (1.1) and (1.2). The gains $K_{1 i j}, K_{2 i j}$ have been moved to the input vector from the output vector so that adjustments in their value do not directly change $u$, i.e. a bumpless transfer. Consequently, the transfer functions from error $e(s)$ to state variable $x_{2 i j}(s)$ is obtained as $\left(k_{2 i j} s+k_{1 i j} j \hat{\omega}_{i}\right) /\left(s^{2}+\left(j \hat{\omega}_{i}\right)^{2}\right)$. i.e., the second state is the sinusoidal component of the original signal and the first state is its quadrature. While the states are time varying, when the signal parameters are time invariant $\overline{A_{i j}}=\sqrt{x_{1 i j}^{2}(t)+x_{2 i j}^{2}(t)}$ is time invariant as is $\phi_{i}=\arctan \left(x_{2 i j}(t) / x_{1 i j}(t)\right)-j \omega_{i}(t)$.

\subsubsection{Frequency estimation $\left(\hat{\omega}_{i}\right)$}

Since the state variables $x_{1 i 1}$ and $x_{2 i 1}$ are orthogonal to each other then, as with the HHT the derivative of the angle of $x_{1 i 1}+\sqrt{-1} x_{2 i 1}$ is $\omega_{i}$. It can be shown that when $\hat{\omega}_{i}=\omega_{i}$ then in steady state, without noise 


$$
\omega_{i}=\frac{d}{d t} L\left(x_{2 i 1}+\sqrt{-1} x_{1 i 1}\right)
$$

thus

$$
\omega_{i}-\hat{\omega}_{i}=\frac{e\left(K_{2 i 1} x_{1 i 1}-K_{1 i 1} x_{2 i 1}\right)}{x_{1 i 1}^{2}+x_{2 i 1}^{2}}
$$

then an integral controller can be used to update the frequency estimates.

$$
\dot{\hat{\omega}}_{i}=K_{a i} \frac{\left(K_{2 i 1} x_{1 i 1}-K_{1 i 1} x_{2 i 1}\right) e}{x_{1 i 1}^{2}+x_{2 i 1}^{2}}
$$

Hence, we assume that the tuning function $G(s)$ has designed properly, and the feedback gains $\left\{K_{111} K_{211}, \ldots, . K_{1 n m_{n}} K_{2 n m_{n}}\right\}$ fore each internal model have calculated. $x_{1 i j}$ and $x_{2 i j}$ with respect with time varying. Thus a quasi-periodic signal can be decomposed into a sum of narrow band signals $\left\{u_{i j}\right\}=\left\{x_{2 i j}\right\}$ in terms of the state variables corresponding to each internal mode and a real time Fourier representation of the reference can be obtained. The signal $u_{i j}(t)$ is the estimate of the signal of interest of the internal models $I M_{i, j}$, and can be represented by

$$
\begin{gathered}
u(t)=\sum_{i=1}^{n} \sum_{j=1}^{m_{i}} u_{i j}(t) \\
u_{i j}(t)=x_{2 i j}(t) \\
\hat{A}_{i j}=\sqrt{\hat{x}_{1 i j}^{2}(t)+\hat{x}_{2 i j}^{2}(t)}
\end{gathered}
$$

Now once the fundamental frequency $\omega_{i}$ match nominal frequency $j \hat{\omega}_{i}$ or become closer, the error $e$ that is started high can be driven to zero. In addition, the steady state solution become as $u_{i j}=\hat{A}_{i j} \sin \left(\hat{\omega}_{i j} t+\varphi_{i j}\right)$.

In [7], it is established for sufficiently small $K_{a i}$ the algorithm is locally exponentially stable when $G(s)$ and the $K_{1 i j}, K_{2 i j}$ are chosen so that the feedback loop in Fig. 3.2 is stable at each point in time. Designing these controller parameters is a challenging problem as it is assumed that 
there is limited knowledge about the $\left\{\omega_{i}\right\}$ and during transients there can be a significant difference between $\left\{\omega_{i}\right\}$ and $\left\{\hat{\omega}_{i}\right\}$.

\subsection{Control parameter selection}

\subsubsection{Off-line tuning}

As with ref [50], we satisfy the above stability assumption by designing the closed loop system to incorporate a bandpass filter with notch filter. Let a 2 nd order desirable bandpas filter be given by

$$
T_{b p}(s)=\frac{d_{1} s^{2}}{s^{4}+c_{1} s^{3}+c_{2} s^{2}+c_{3} s+c_{4}}
$$

We choose the controller parameters to be such that the transfer function from $d$ to $e$

$$
\begin{gathered}
T_{d e}=\frac{d_{1} s^{2}}{s^{4}+c_{1} s^{3}+c_{2} s^{2}+c_{3} s+c_{4}} \times \\
\prod \frac{s^{2}+\left(j \hat{\omega}_{i}\right)^{2}}{s^{2}+2 \epsilon_{i j} j \hat{\omega}_{i} s+\left(j \hat{\omega}_{i}\right)^{2}}
\end{gathered}
$$

where $\epsilon_{i j}$ are small real numbers, and $j \hat{\omega}_{i}$ are the notches frequency. The presence of the numerator of the second term is a fundamental consequence of the internal model principle. Therefore, the ability of the algorithm to improve noise rejection is achieved. 


\section{CHAPTER 3. ALGORITHM DEVELOPMENT}

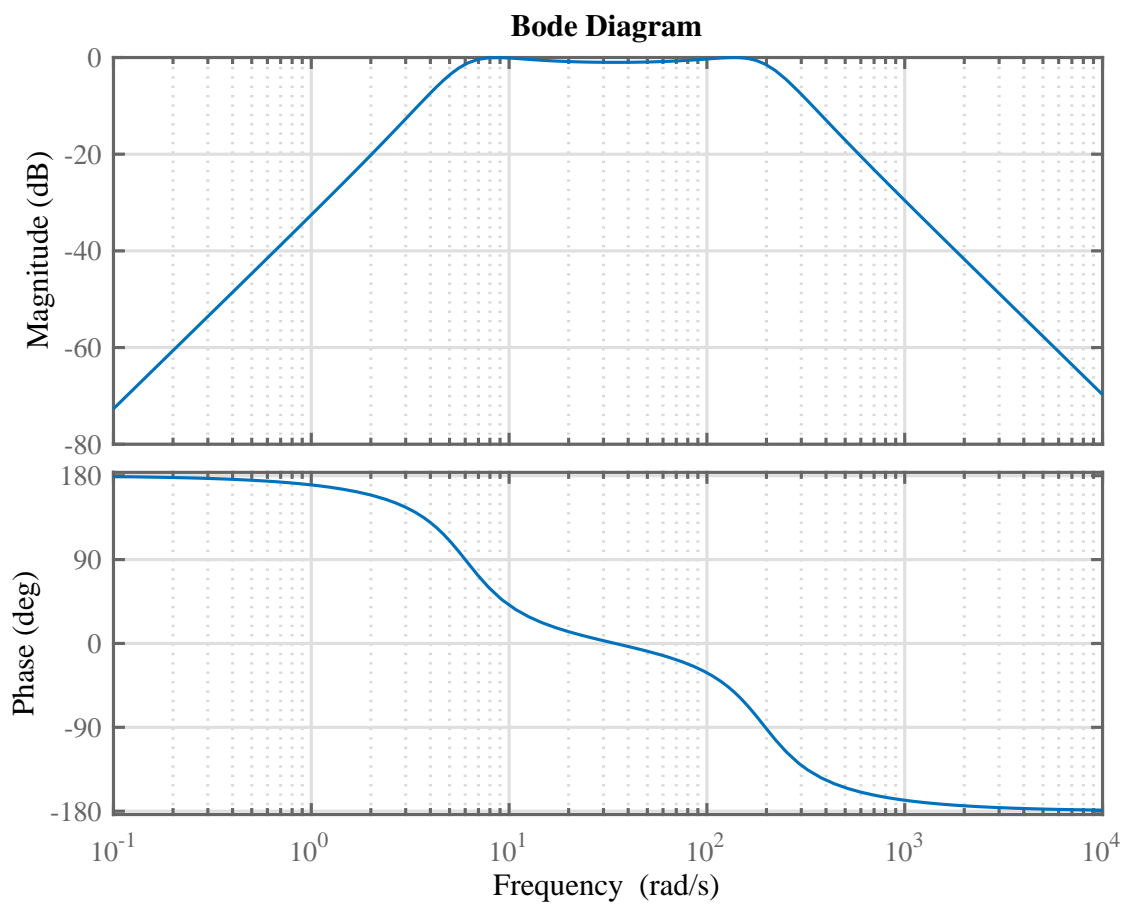

Figure 3.3: $T_{b p}$ bode plot diagram

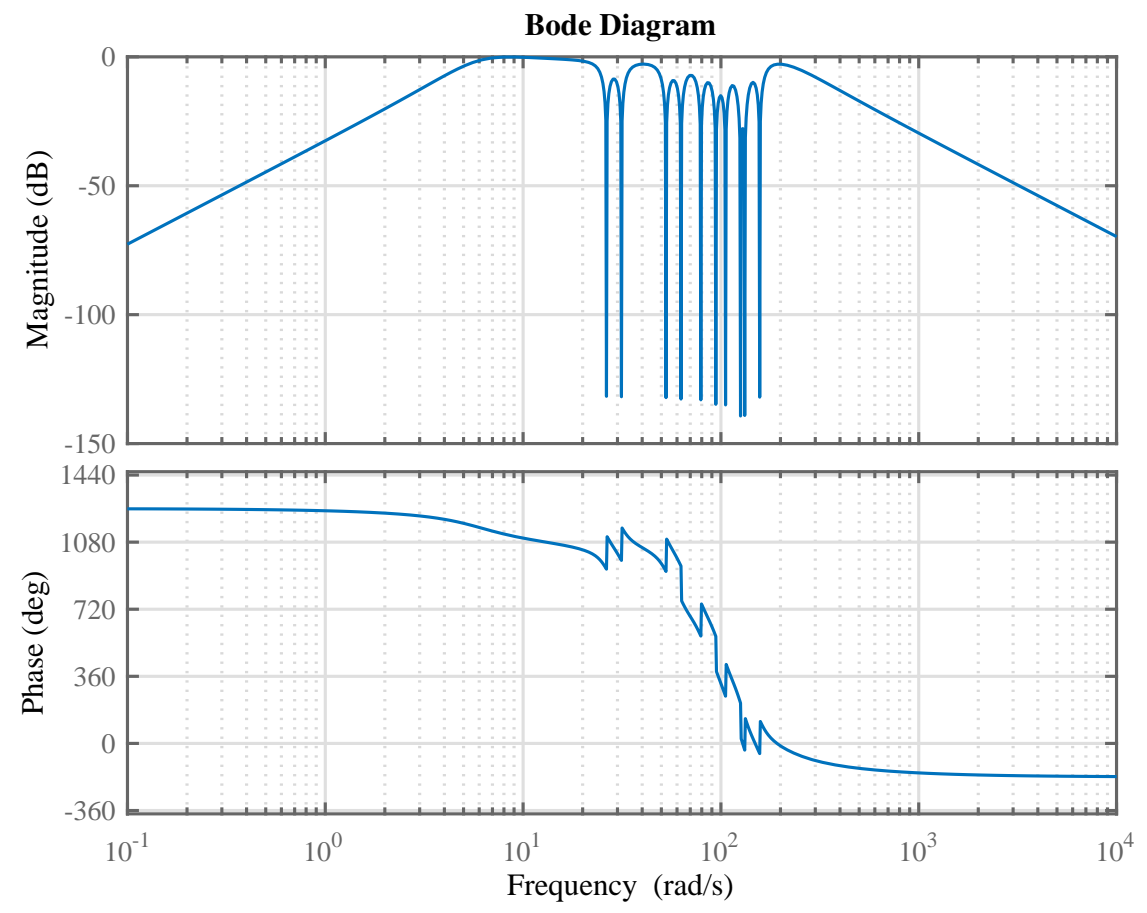

Figure 3.4: $T_{b p n}$ bode plot diagram 
Fig. (3.3) and Fig. (3.4) show the theoretical bode plot of the desired bandpass filter and the bandpass with notches filter respectively. The frequency response of bandpass filter with 10 notches (dynamic system model) can be seen in Fig. 3.4.

An analysis of Fig 3.2 gives

$$
\begin{aligned}
T_{d e} & =\frac{G(s)}{1+G(s) \sum_{i=1}^{n} \sum_{j=1}^{m_{i}}\left(\frac{K_{2 i j} s+K_{1 i j} j \hat{\omega}_{i}}{s^{2}+\left(j \hat{\omega}_{i}\right)^{2}}\right)} \\
& =\frac{b_{1} s^{2} \prod\left(s^{2}+\left(j \hat{\omega}_{i}\right)^{2}\right)}{a(s) \prod\left(s^{2}+\left(j \hat{\omega}_{i}\right)^{2}\right)+b_{1} s^{2} \sum\left(K_{2 k l} s+K_{1 k l} l \hat{\omega}_{k}\right) \gamma_{k l}(s)}
\end{aligned}
$$

where

$$
\begin{gathered}
\gamma_{k l}=\prod_{i=1}^{n_{i}} \prod_{j=1}^{m_{i}}\left(s^{2}+\left(j \hat{\omega}_{i}\right)^{2}\right) \\
\{j \neq l \text { if } i=k\}
\end{gathered}
$$

Note in equation (3.18) and (3.19) $\prod$ represents $\prod_{i=1}^{n} \prod_{j=1}^{m_{i}}$ and $\sum$ represents $\sum_{k=1}^{n} \sum_{l=1}^{m_{k}}$. The terms $\gamma_{k l}$ are the product of all the terms $s^{2}+\left(j \hat{\omega}_{i}\right)^{2}$ except the $i=k, j=l$ term.

More details of how we designed band-pass filter with multi notches, and how the parameters $b 1, a_{i}$ and $\left\{K_{111} K_{211}, \ldots, K_{1 n m_{n}} K_{2 n m_{n}}\right\}$ are calculated can be found in appendix A.2. Now, all the controller parameters can be calculated by matching the coefficients of numerator and denominators in equations (3.18) and (3.19). Note, the only controller parameters in the numerator is $b_{1}$ hence we get $b_{1}=d_{1}$. A unique solution for $a_{i}$, where $i=(1,2, \ldots, 4)$ for the tuning function $G(s)$ and the feedback gains $K_{p}=\left\{K_{111} K_{211}, \ldots, . K_{1 n m_{n}} K_{2 n m_{n}}\right\}$ for each internal model can be derived from the denominator.

Unfortunately we get a set of $2 n_{t}+4$ coupled equations with $2 n_{t}+4$ unknowns where $n_{t}=$ $\sum_{i=1}^{n} m_{i}$, which is possible to solve off-line but not possible to solve in real time. The contribution of this thesis is to develop a less computationally intensive algorithm for calculating the controller parameter to meet the real time requirement. 


\subsubsection{Online controller gain selection}

The crucial question is how to choose $G(s)$ and $K_{p}$ and implement the algorithm without needing to set up and solve a set or $2 n_{t}+4$ linear equations. It can be seen that all of the terms in the denominator except the term containing $\gamma_{k l}$ will be zero if $s= \pm \sqrt{-1} l \hat{\omega}_{k}$. Thus when $s= \pm \sqrt{-1} l \hat{\omega}_{k}$

$$
\begin{gathered}
b_{1} s^{2}\left(K_{2 k l} s+K_{1 k l} l \hat{\omega}_{k}\right) \gamma_{k l}(s) \\
=\left(s^{4}+c_{1} s^{3}+c_{2} s^{2}+c_{3} s+c_{4}\right) \prod \prod\left(s^{2}+2 \epsilon_{i j} j \hat{\omega}_{i} s+\left(j \hat{\omega}_{i}\right)^{2}\right)
\end{gathered}
$$

This generates 2 complex and complementary conjugate equations with 2 unknowns i.e., the real part of either equation gives $K_{1 i j}$ and the imaginary gives $K_{2 i j}$ Solving these two linear equations the values of the control feedback gains parameters $K_{1 i j}, K_{2 i j}$ for each internal model $I M_{i, j}$ can be obtained. The $4 a_{i}$ parameters can then be explicitly solved by equating the coefficients of the degree $0,1,2 n_{t}+2,2 n_{t}+3$ terms of the denominator. Note the second term of the denominator of eq (3.19) contribute nothing to these four terms. In ref [22] J. Chen found out that these coefficients can be calculated by utilizing the relationships between the coefficients of a polynomial and the roots of a polynomial. We have that.

$$
\begin{aligned}
\prod_{i=0}^{n_{t}}\left(s+r_{i}\right)= & s^{n_{t}}+\sum r_{i} s^{n_{t}-1}+\sum_{i} \sum_{j>i} r_{i} r_{j} s^{n_{t}-2} \\
& +\cdots+s \sum_{i} \prod_{j \neq i} r_{j}+\prod_{i} r_{i} .
\end{aligned}
$$

Extending this to the following product 


$$
\begin{aligned}
\prod_{i=1}^{n_{t}}\left(s^{2}+2 \epsilon_{i} \mathrm{~W}_{i}+\mathrm{w}_{i}^{2}\right) & =s^{2 n_{t}}+\sum 2 \epsilon_{i} \mathrm{w}_{i} s^{2 n_{t}-1} \\
& +\left(\sum \mathrm{w}_{i}^{2}+\sum_{i} \sum_{j>i} 4 \epsilon_{j} \mathrm{w}_{j} \epsilon_{i} \mathrm{~W}_{i}\right) s^{2 n_{t}-2} \\
& +\cdots+s \sum_{i} 2 \epsilon_{i} \mathrm{~W}_{i} \prod_{j \neq i} \mathrm{w}_{j}^{2}+\prod \mathrm{w}_{i}^{2}
\end{aligned}
$$

and equating the following sets $\left\{\mathrm{w}_{i}\right\}=\left\{j \omega_{i}\right\}$ and $\left\{\epsilon_{i}\right\}=\left\{\epsilon_{i j}\right\}$, we get

$$
\begin{aligned}
& a_{1}=c_{1}+\sum 2 \epsilon_{i} \mathrm{w}_{i} \\
& a_{2}=\sum_{i=1}^{n_{t}} \sum_{j>i} 4 \epsilon_{j} \mathrm{w}_{j} \epsilon_{\mathrm{i}} \mathrm{w}_{i}+c_{1} \sum_{i=1}^{n_{t}} 2 \epsilon_{i} \mathrm{w}_{i}+c_{2} \\
& a_{3}=c_{3}+c_{4} \sum_{i=1}^{n_{t}} 2 \epsilon_{i} / \mathrm{w}_{i} \\
& a_{4}=c_{4}
\end{aligned}
$$

\subsubsection{Linear dependency of the equations}

Note however, if $j \hat{\omega}_{i}=l \hat{\omega}_{k}$ then the denominator of equation (A.8) will be zero and it will not be possible to calculate two pairs of internal model gains. In other words, the the equations become linearly dependent with our solution technique this is reflected in the fact that denominator of equation (3.19) will be zero when we substitute in $s= \pm \sqrt{-1} l \hat{\omega}_{k}$. Further, while it is theoretical possible to solve when the frequencies are extremely close, we get solutions that lead to unstable results because of numerical stability issues.. To solve this problem, while calculating the controller gains, we drop the approximately redundant internal model when the frequencies become close, i.e. within $0.01 \%$. After calculating the controller gains, the two redundant models are each assigned half of the gain. That is when $j \hat{\omega}_{i}=l \omega_{k}$, we drop Internal model $I M_{l, k}$ from the design stage. Let $\overline{K_{1 i j}}$ and $\overline{K_{2 i j}}$ be the calculated controllers gains. Then $K_{1 i j}=K_{1 k l}=0.5 \overline{K_{1 i j}}$ and $K_{2 i j}=K_{2 k l}=0.5 \overline{K_{2 i j}}$. It should be noted that the threshold for HHT to distinguish between close frequencies is $10 \%$. 


\subsection{Alternative approach for choosing suitable coefficients of the system controller}

We showed how our desired system behave as bandpass filter with notches and it's main advantage to estimate the instantaneous frequencies and noise rejection of the periodic signal although it allows the difference between $\mathrm{d}$ and $\mathrm{u}$ to have a substantial DC content. Therefore, we have replaced the chebyshev bandpass filter in figure (3.2) by lowpass filter as shown in figure (3.5), so the order of the system controller has been reduced from fourth order to second with one unknown parameter instead of four. More precisely, in this alternative method, It is necessary to remove the low frequency content from the input to the internal models in order for correct estimation of the signal frequencies. The closed loop poles associated with this low frequency cutoff determine the speed of the system. Thus, to improve controller system performance, the low frequency components can be estimated by an additional internal model and the bandpas filter replace by a low pass filter. The faster poles of the lowpass filter result in a faster system and better performance.

Our goal here is to improve the performance of the proposed algorithm by identifying the DC as well as the first and the second components; thus, the actual outputs of the signal generators become much closer to the identified signals by eliminating the DC component. Corresponding to the Fig. (3.5) the feedback system is an integral in parallel with the other embedded internal models. Such that a second order Chebyshev lowpass filter $T_{L p}$ can be given by

$$
T_{L p}(s)=\frac{b}{s^{2}+d s+c}
$$

In figure (3.6) it can be seen the system has a constant magnitude at low frequency before it reached the cutoff frequency $182.2124 \mathrm{rad} / \mathrm{s}$ while the gain started to decrease slowly and then sharply drooped down to eliminate the high frequency from passing trough it. In other words, the slowest poles are located at highpass cutoff instead of the lowpass cutoff in the bandpass filter.

When the controller parameters of the control system have been calculated, then the transfer function of lowpass filter with notches become as 


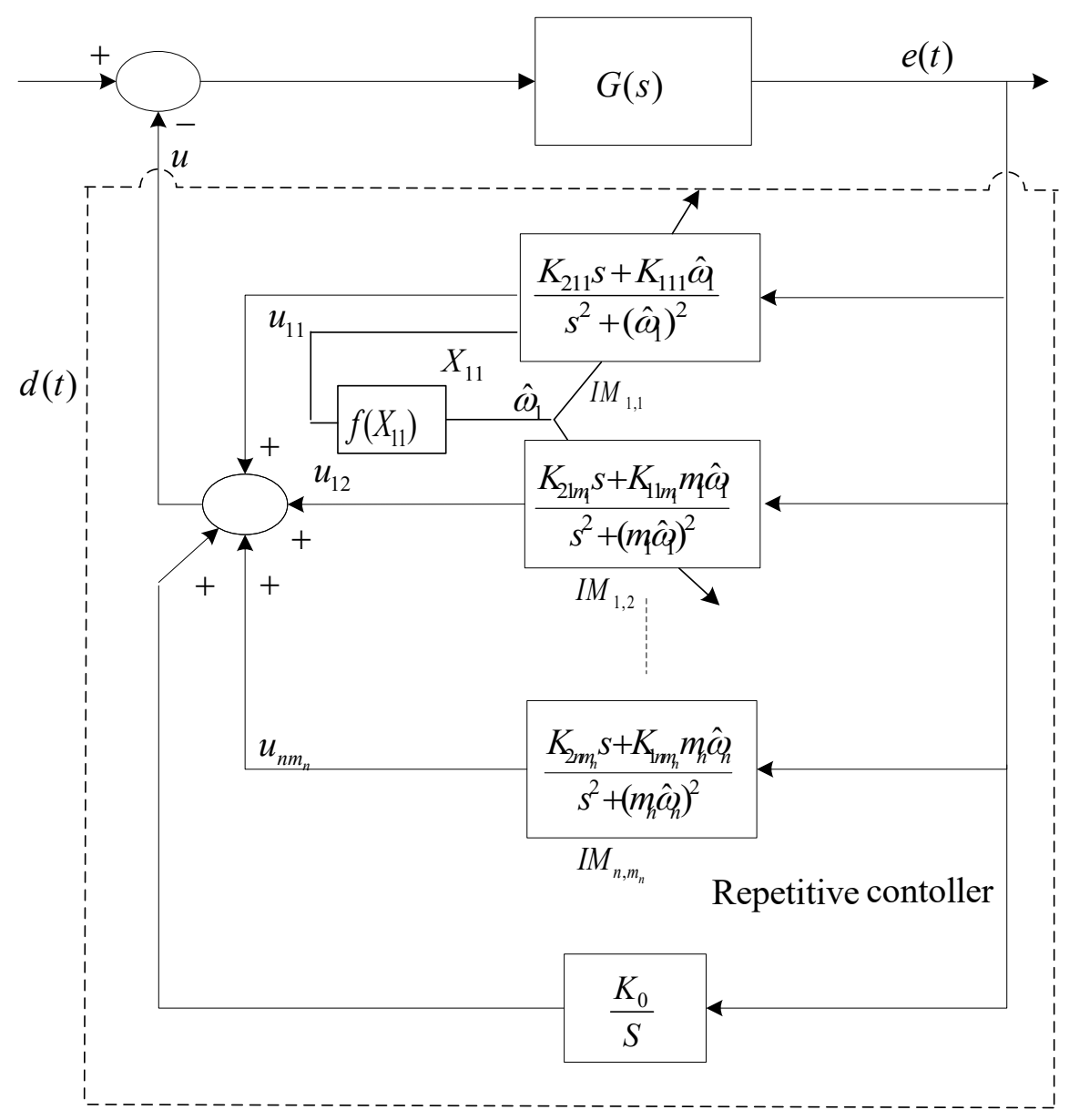

Figure 3.5: Structure of alternative proposed instantaneous Fourier decomposition

$$
T_{L p n}=\frac{b}{s^{2}+d s+c} \times \prod \frac{s^{2}+\left(j \hat{\omega}_{i}\right)^{2}}{s^{2}+2 \epsilon_{i j} j \hat{\omega}_{i} s+\left(j \hat{\omega}_{i}\right)^{2}}
$$

The tuning function $G(s)$ has been reduced from five unknown coefficients (in our first proposed tuning function) to two, $a$ and $\bar{b}$ given by

$$
G(s)=\frac{\bar{b}}{s+a}
$$

and the transfer function of the closed loop feedback system can be presented as 


\section{CHAPTER 3. ALGORITHM DEVELOPMENT}

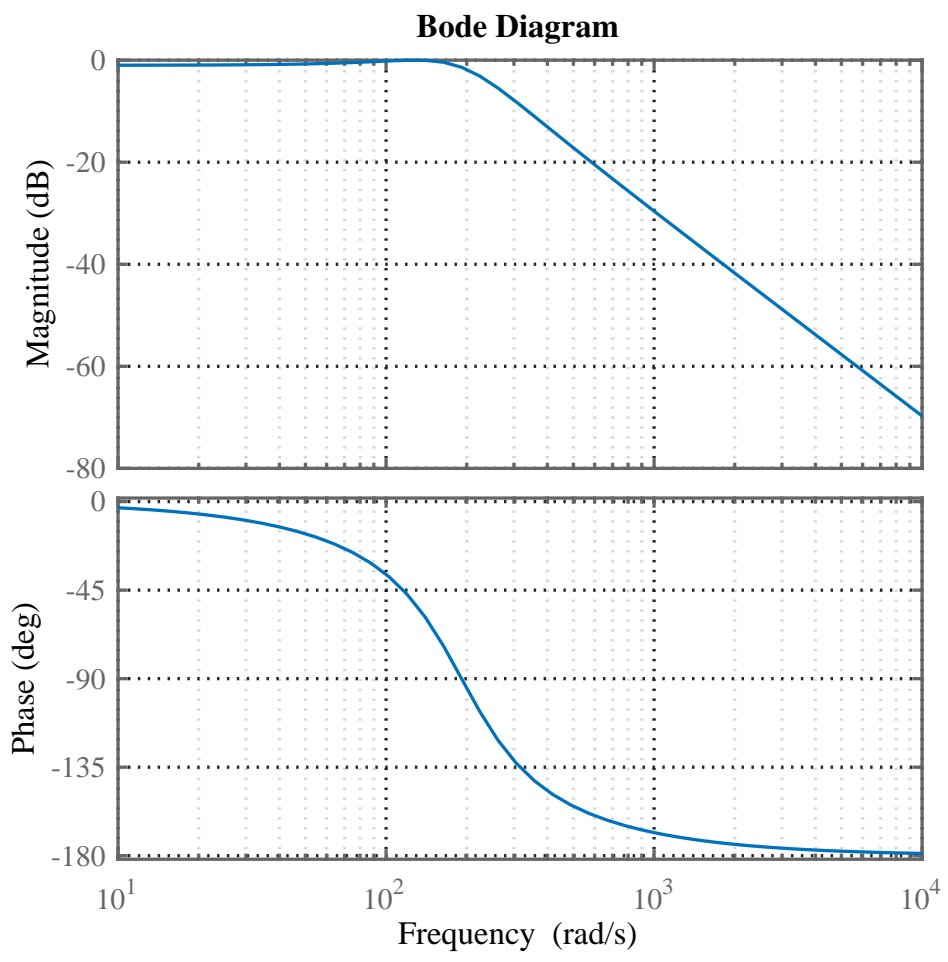

Figure 3.6: $T_{L p}$ bode plot diagram

$$
\begin{aligned}
T_{d e} & =\frac{G(s)}{1+G(s) \sum_{i=1}^{n} \sum_{j=1}^{m_{i}}\left(\frac{K_{2 i j} s+K_{1 i j} j \hat{\omega}_{i}}{s^{2}+\left(j \hat{\omega}_{i}\right)^{2}}+\frac{K_{0}}{s}\right)} \\
& =\frac{\bar{b} s \prod\left(s^{2}+\left(j \hat{\omega}_{i}\right)^{2}\right)}{(s+a) \prod\left(s^{2}+\left(j \hat{\omega}_{i}\right)^{2}\right) s+\bar{b} s \sum\left(K_{2 k l} s+K_{1 k l} l \hat{\omega}_{k}\right) \gamma_{k l}(s)+\bar{b} K_{0} \prod\left(s^{2}+\left(j \hat{\omega}_{i}\right)^{2}\right)}
\end{aligned}
$$

where

$$
\begin{gathered}
\gamma_{k l}=\prod_{i=1}^{n_{i}} \prod_{j=1}^{m_{i}}\left(s^{2}+\left(j \hat{\omega}_{i}\right)^{2}\right) \\
\{j \neq l \text { if } i=k\}
\end{gathered}
$$




\subsubsection{Alternative approach controller parameters calculation}

We do exactly the same procedure (same theory) that we did in our proposed algorithm that given in $[22,31]$. Thus, in order to calculate the feedback control system gains $\left\{K_{111} K_{211}, \ldots, . K_{1 n m_{n}} K_{2 n m_{n}}\right\}$ for each internal models, we do match the coefficients of numerators and denominators in equations (3.22) and (3.24). Therefore, equating the numerators gives $\bar{b}=b$, and when $s= \pm \sqrt{-1} l \hat{\omega}_{k}$

$$
\begin{gathered}
\bar{b} s\left(K_{2 k l} s+K_{1 k l} l \hat{\omega}_{k}\right) \gamma_{k l}(s) \\
=\left(s^{2}+d s+c\right) \prod\left(s^{2}+2 \epsilon_{i j} j \hat{\omega}_{i} s+\left(j \hat{\omega}_{i}\right)^{2}\right)
\end{gathered}
$$

Similarly when $s=0, K_{0}=b / c$.

Also, the $a$ coefficient for the tuning function $G(s)$ can be calculated by equating the coefficients of the degree $n+1$ term of the denominator. Thus, this generate a linear equation which can be solved by back substitution

$$
a=d+\sum_{i=1}^{n_{t}} 2 \epsilon_{i} \mathrm{w}_{i}
$$

\subsubsection{State Space Form of (IFD) within discrete time}

Similar to what we did in the continuous time, the instantaneous decomposition series state space in (3.1) is converted from continuous time to the discrete state space model. This idea was first proposed by L. J. Brown and Z. Zhao in 2004 [53] and then modified by Q. Zhang and Y. Ma in 2004 and 2009 respectively. According to Y. Ma "The technique is a band-pass filter bank which enables randomly number and spacing of channels. Therefore, this approach has computational complexity of $O(L . N)$, the same order as sliding FFT and as low as theoretical limit." [28].

So, each internal model in equation (3.9) can be rewritten and expressed in discrete state space by

$$
\dot{X}_{i j}(k+1)=A_{d i j} X_{i j}(k)+B_{d i j} e(k)
$$


where

$$
\begin{gathered}
A_{d i j}=\left[\begin{array}{cc}
\cos \left(j \hat{\omega}_{i}\right) & -\sin \left(j \hat{\omega}_{i}\right) \\
\sin \left(j \hat{\omega}_{i}\right) & \cos \left(j \hat{\omega}_{i}\right)
\end{array}\right] \quad B_{d i j}=\left[\begin{array}{c}
\bar{K}_{1 i j} \\
\bar{K}_{2 i j}
\end{array}\right]=\left[\begin{array}{c}
\frac{K_{1 i j} \sin j \hat{\omega}_{i}}{j \hat{\omega}_{i}}+\frac{K_{2 i j} \cos j \hat{\omega}_{i}}{j \hat{\omega}_{i}} \\
\frac{-K_{1 i j} \cos j \hat{\omega}_{i}}{j \hat{\omega}_{i}}+\frac{K_{2 i j} \sin j \hat{\omega}_{i}}{j \hat{\omega}_{i}}
\end{array}\right] \\
u_{i j}(k)=\left[\begin{array}{ll}
0 & 1
\end{array}\right] X_{i j}(T) \\
X_{i j}(k)=\left[x_{1 i j}(k) x_{2 i j}(k)\right]^{T}
\end{gathered}
$$

Identical to equations (3.12) and (3.16). Where $\omega_{i}(k)$ and $A_{d i j}(k)$ are adaptive frequency and the magnitude respectively and they can be computed as follows

$$
\begin{gathered}
A_{d i j}(k)=\sqrt{x_{1 i j}^{2}(k)+x_{2 i j}^{2}(k)} \\
\Delta \omega_{i}(k)=\omega_{i}(k+1)-\hat{\omega}_{i}(k)=-K_{a} \frac{e\left(K_{2 i 1} x_{1 i 1}-K_{1 i 1} x_{2 i 1}\right)}{x_{1 i 1}^{2}+x_{2 i 1}^{2}}
\end{gathered}
$$

The transfer function for each internal model $\left(I M_{i j}\right)$ from error $e(k)$ to the variable state spaces $x_{2 i j}(k)$ in the discrete form can be expressed by

$$
\frac{\bar{K}_{2 i j} z-\bar{K}_{2 i j} \cos \left(j \hat{\omega}_{i}\right)+\bar{K}_{1 i j} \sin \left(j \hat{\omega}_{i}\right)}{z^{2}-2 z \cos \left(j \hat{\omega}_{i}\right)+1}=\frac{K_{2 i j} z+\overline{\bar{K}}_{1 i j}}{z^{2}-2 z \cos \left(j \hat{\omega}_{i}\right)+1} .
$$

where,

$$
\begin{aligned}
K_{1 i j} & =j \omega_{i}\left(j \hat{\omega}_{i} \bar{K}_{1 i j} \sin j \hat{\omega}_{i}+\bar{K}_{2 i j} j \hat{\omega}_{i} \cos j \hat{\omega}_{i}\right) \\
K_{2 i j} & =j \hat{\omega}_{i} \bar{K}_{2 i j} \sin j \hat{\omega}_{i}+\bar{K}_{1 i j} j \hat{\omega}_{i} \cos j \hat{\omega}_{i} \\
\bar{K}_{1 i j} & =\frac{\overline{\bar{K}}_{1 i j}+\overline{\bar{K}}_{2 i j} \cos j \hat{\omega}_{i}}{\sin j \hat{\omega}_{i}}
\end{aligned}
$$

Same procedure that is done in continuous time can be repeated here in the discrete time. Tuning of the algorithm is focused on the transfer function from input signal to the error signal $T_{d e}(z)$. Therefore, the tuning transfer function $G(z)$ and a second order band-pass filter with notches $T_{b p n}(z)$ are represented in discrete time as follows 


$$
T_{b p}(z)=\frac{\operatorname{Num}(z)}{\operatorname{Den}(z)}=\frac{n_{1} z^{4}+n_{2} z^{3}+n_{3} z^{2}+n_{4} z+n_{5}}{z^{4}+d_{1} z^{3}+d_{2} z^{2}+d_{3} z+d_{4}}
$$

The controller parameters are chosen to be such that the transfer function from $d$ to $e$ presented by

$$
\begin{aligned}
& T_{b p n}(z)=\frac{n_{1} z^{4}+n_{2} z^{3}+n_{3} z^{2}+n_{4} z+n_{5}}{z^{4}+d_{1} z^{3}+d_{2} z^{2}+d_{3} z+d_{4}} \times \\
& \prod_{k=1}^{n} \prod_{l=1}^{m_{i}} \frac{\left(z-e^{l \hat{\omega}_{k} \sqrt{-1}}\right)\left(z-e^{-l \hat{\omega}_{k} \sqrt{-1}}\right)}{\left(z-e^{-l \hat{\omega}_{k} \varepsilon_{k l}+j \hat{w}_{k} l} \sqrt{1-\epsilon_{k l}^{2}}\right)\left(z-e^{-l \hat{\omega}_{k} \epsilon_{k l}-j \hat{\omega}_{k} l} \sqrt{1-\epsilon_{k l}^{2}}\right)}
\end{aligned}
$$

alternatively

$$
\begin{aligned}
& T_{b p n}(z)=\frac{n_{1} z^{4}+n_{2} z^{3}+n_{3} z^{2}+n_{4} z+n_{5}}{z^{4}+d_{1} z^{3}+d_{2} z^{2}+d_{3} z+d_{4}} \times \\
& \prod_{k=1}^{n} \prod_{l=1}^{m_{i}} \frac{\left(z^{2}-2 z \cos l \hat{\omega}_{k}+1\right) \cdot e^{l \hat{\omega}_{k} \epsilon_{k l}}}{z^{2} e^{l \hat{\omega}_{k} \epsilon_{k l}}-2 z \cos \left(\hat{\omega}_{k} l \sqrt{1-\epsilon_{k l}^{2}}\right)+e^{-l \hat{\omega}_{k} \epsilon_{k l}}}
\end{aligned}
$$

where $i=k, j \neq l, \epsilon_{k l}$ are small real numbers, and $l \hat{\omega}_{k}$ is the notches frequency. Therefore, the transfer function of the feedback system is represented as follows.

$$
T_{d e}(z)=\frac{G(z)}{1+G(z) \sum_{i=1}^{n} \sum_{j=1}^{m_{i}}\left(\frac{\bar{K}_{2 i j} z-\bar{K}_{1 i j} \sin j \hat{\omega}_{i}+\bar{K}_{2 i j} \cos j \hat{\omega}_{i}}{z^{2}-2 \cos j \hat{\omega}_{i} z+1}\right)}
$$

and

$$
G(z)=\frac{b_{1} z^{4}+b_{2} z^{3}+b_{3} z^{2}+b_{4} z+b_{5}}{z^{4}+a_{1} z^{3}+a_{2} z^{2}+a_{3} z+a_{4}}=\frac{b(z)}{a(z)}
$$

The previous work and the approach that used to calculate system parameters are explained more in appendix B. 


\subsection{Summary}

This method is based on the behavior of the principle model controller with adaptive parameters. At the beginning of this chapter we showed how the parameters are calculated in the and implemented in the offline in the previoues work and the limitations that has; further, in this chapter, the adaptive (IFD) algorithm has been modified in both continuous and discrete time forms so the dynamics of the closed loop system can be selected by the user and can be successfully implemented in realtime. As a result of our work here in this chapter especially in sec. 3.2.2, the algorithm has been successfully implemented online after solving for the issue of overlapping harmonics from different signal components. One of the models is removed from the design process eliminating the dependent equations. The associated controller gains are distributed equally in the implemented controller. Moreover, an alternative approach has been proposed, and coefficients of feedback close loop system calculation have been shown as well in sec 3.3. 


\section{Chapter 4}

\section{Simulation Results and Comparison}

\subsection{Simulation and Comparison}

In this particular chapter, we are going to show not only the effectiveness of the proposed adaptive (IFD) algorithm but also the comparison results with other algorithms, such as (HHT) using (EMD). In addition, we are going to compare the simple tuning function shown in Fig.3.1 with the proposed tuning function given in Fig. 3.2 as well as the alternative proposed approach shown in figure 3.5. Thus, the performance of our adaptive algorithm is examined via matlab and simulink. The model configuration parameters that are used with the Matlab (R2016a) environment are as follows: Solver ode5 (Dormand-prince) selection with fundamental sample time 0.0025s. Thus, the sampling rate in our case was selected to be $400 \mathrm{~Hz}$, while the Nyquist frequency was $200 \mathrm{~Hz}$. All random numbers were zero mean.

\subsubsection{Computing a Tuning Function}

Our signal to be identified was produced by summing the outputs of two copies of the model shown in Fig. 4.1. The feedback loop containing the pure delay $\left(e^{-s T}\right)$ is called a repetitive controller and is capable of producing any periodic disturbances with period T. The frequency $\mathrm{f}$ was an integrated band limited white and was converted to a period by the block $1 / x$. The frequency band of this noise 


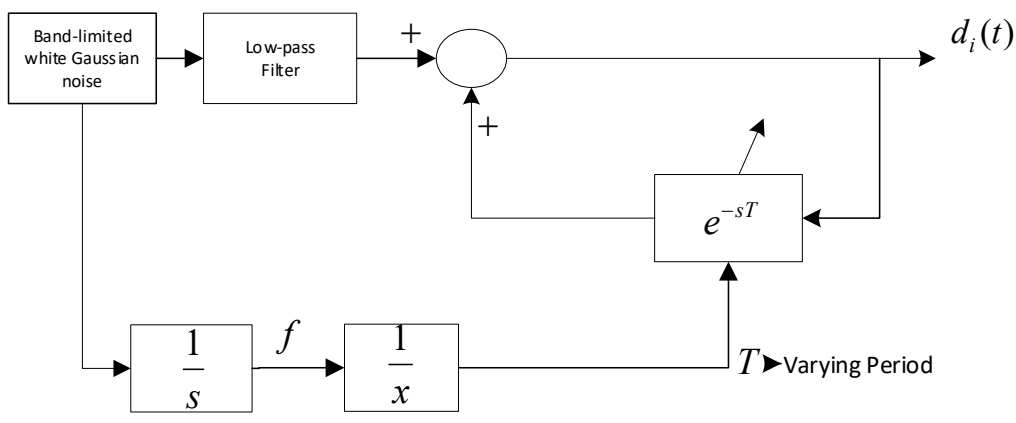

Figure 4.1: Block diagram of periodic signals generator

was $20 \mathrm{rad} / \mathrm{s}$ and the variance was 0.5 . The initial conditions for both fundamental frequencies $\omega_{1}$ and $\omega_{2}$ were chosen to be $(26.3894,31.4159) \mathrm{rad} / \mathrm{s}$ respectively. The disturbance input to the repetitive controller causes the amplitudes and relative phases to vary slowly with time as well. In addition measurement noise was added to the sums of the two signals produced by two copies of Fig. 4.1. This random signal was bandlimited to $50 \mathrm{~Hz}$ and had a variance of 0.1 . The low pass filter had a cutoff frequency of $100 \mathrm{rad} / \mathrm{s}$ concentrating the energy in the harmonics to below the fourth and third harmonic respectively though the signal was present in all harmonics up to the Nyquist frequency.

Figure 4.2 shows snapshots of the signal to be identified at two times. We can see that in the first time the signal has a regular structure that appears to repeat every $7 / 6$ of a second. However, in the next snapshot that earlier structure is diferent repeating every $2.5 \mathrm{~s}$. We can also seem small variations within the repeating structure. 

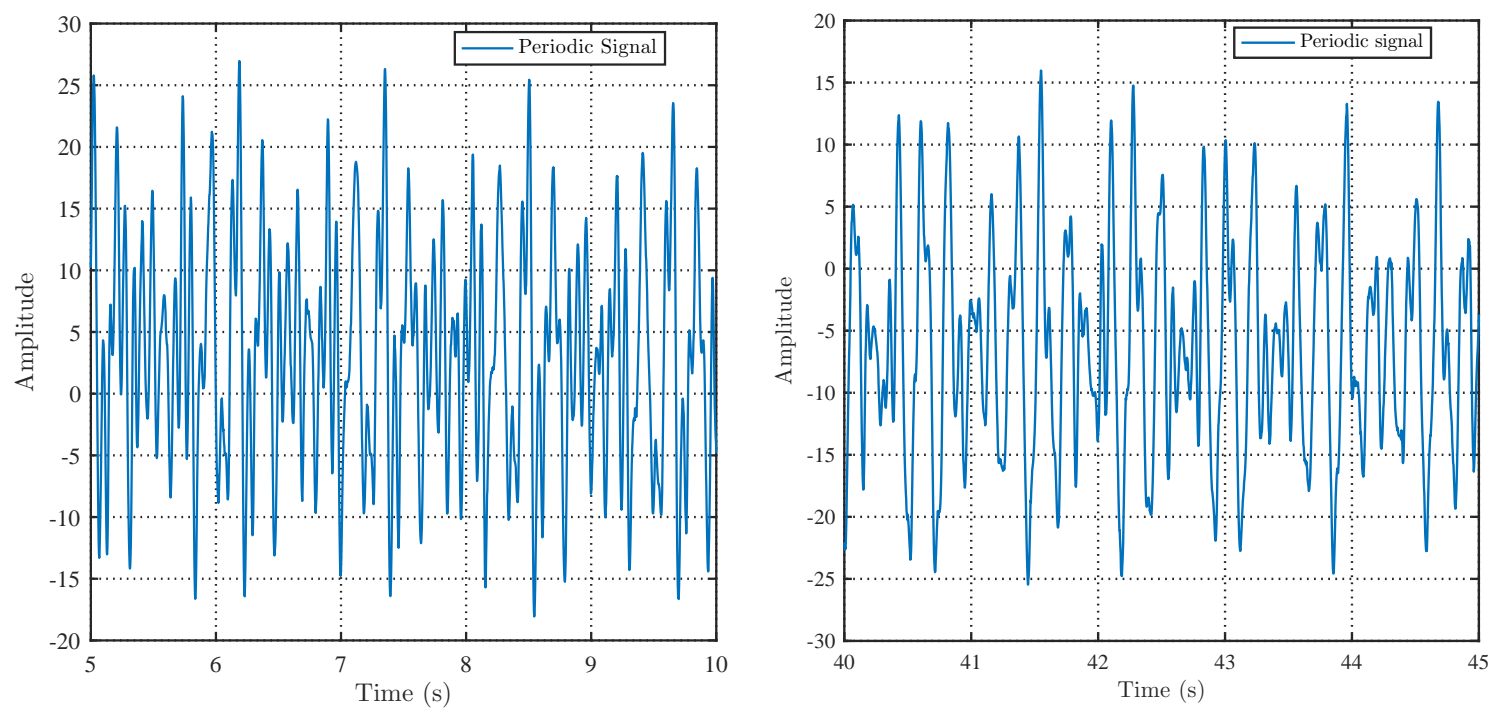

Figure 4.2: Periodic Disturbance

The frequency adaption gains were chosen as $K_{a 1}=1.95$ and $K_{a 2}=1.95$ or with frequency $7.5 \%$ to $10 \%$ of the fundamental frequencies. A second order Chebyshev bandpass filter was designed with a $1 \mathrm{~dB}$ bandpass ripple, and low and high bandpass frequencies of 1 and $30 \mathrm{~Hz}$, respectively as given in Table. 4.1 .

\begin{tabular}{|c|c|c|c|c|}
\multicolumn{5}{|c|}{ Bandpass Filter Parameters } \\
\hline \hline$d_{1}=b_{1}$ & $c_{1}$ & $c_{2}$ & $c_{3}$ & $c_{4}$ \\
\hline $3.2624 \cdot 10^{04}$ & 200.0208 & $3.8974 \cdot 10^{4}$ & $2.3690 \cdot 10^{5}$ & $1.4027 \cdot 10^{6}$ \\
\hline
\end{tabular}

Table 4.1: Bandpass filter parameters

For initial values of $\hat{\omega}_{1}=4.2 * 2 * \pi$ and $\hat{\omega}_{2}=5 * 2 * \pi$ and $\epsilon_{i j}=.1$ the controller gains are given in Table 4.1

The tuning function $G(s)=b_{1} s^{2} /\left(s^{4}+a_{1} s^{3}+a_{2} s^{2}+a_{3} s+a_{4}\right)$ is properly designed to guarantee the stability of the control system. The number of the internal model $I M_{i, j}$ connected in parallel with the tuning function is the same as the number of notches filter. Therefore, the sums of $n$ periodic components with each component composed of $m_{i}$ harmonics is the same as the number of internal models in the estimation system with transfer function $\left(s^{2}+\left(j \hat{\omega}_{i}\right)^{2}\right) /\left(s^{2}+2 \epsilon_{i j} j \hat{\omega}_{i} s+\left(j \hat{\omega}_{i}\right)^{2}\right)$ 
where $i=(1,2, \ldots, n)$. The coefficients of the simple tuning function are given in the Table 4.2

Tuning Function Parameters G(s)

\begin{tabular}{|c|c|c|c|c|}
\hline \hline$b_{1}$ & $a_{1}$ & $a_{2}$ & $a_{3}$ & $a_{4}$ \\
\hline $3.2624 \cdot 10^{04}$ & 373.4367 & $8.6845 \cdot 10^{04}$ & $2.8156 \cdot 10^{05}$ & $1.4027 \cdot 10^{06}$ \\
\hline
\end{tabular}

Table 4.2: Values of simple tuning function $\mathrm{G}(\mathrm{s})\left(b_{1}, a_{1}, a_{2}, a_{3}\right.$ and $\left.a_{4}\right)$.

so the tuning function can be simplified as

$$
G(s)=\frac{3.2624 \cdot 10^{04} s^{2}}{s^{4}+373.4367 s^{3}+8.6845 \cdot 10^{04} s^{2}+2.8156 \cdot 10^{05} s+1.4027 \cdot 10^{06}}
$$

and the values of feedback gains $\left\{K_{111} K_{211}, \ldots, . K_{1 n m_{n}} K_{2 n m_{n}}\right\}$ calculated offline can be found in the table. 4.3

Values Of $K_{p}$

\begin{tabular}{|c|c|c|c|}
\hline \hline$i$ & $j$ & $k_{1 i j}$ & $k_{2 i j}$ \\
\hline 1 & 1 & -2.6141 & 2.2577 \\
\hline 1 & 2 & -7.7969 & 10.1378 \\
\hline 1 & 3 & -21.8565 & 4.9823 \\
\hline 1 & 4 & -18.2360 & 35.7454 \\
\hline 1 & 5 & 59.1736 & 55.2486 \\
\hline 2 & 1 & -0.0733 & 8.3566 \\
\hline 2 & 2 & -1.3070 & 1.1288 \\
\hline 2 & 3 & -32.1937 & 12.3257 \\
\hline 2 & 4 & -69.2218 & 37.8807 \\
\hline 2 & 5 & 21.2844 & 36.9857 \\
\hline
\end{tabular}

Table 4.3: values of $\mathrm{K}$ 


\subsubsection{Frequency identification}

Under these conditions, a $50 \mathrm{~s}$ Matlab simulation could be performed in under $5 \mathrm{~s}$. The identified first and second frequencies are shown respectively in Fig. 4.3 and Fig. 4.4. we can see good identification and tracking of the fundamental frequencies. In Fig. 4.3 the estimated frequency $\hat{\omega}_{i}$ started to converge at $2.46 \mathrm{~s}$, which is good transient time converge of the frequency in real time identification with the initial frequency $4.5 \mathrm{~Hz}$ before it went to the true frequency $4.2 \mathrm{~Hz}$. Moving to Fig. 4.4 that corresponds to our second internal model frequency identification, the estimated frequency converged from $3.5 \mathrm{~Hz}$ to the fundamental frequency value $5 \mathrm{~Hz}$, with transient time response $2.54 \mathrm{~s}$, which is a really fast convergence time. Thus, we can see not only a good identification of the fundamental frequencies but also a lower transient time response with smooth fundamental frequency change by $0.1 \mathrm{~s}$. In other words, high performance tuned algorithm frequency tracking with low level overshoots are achieved. Moving to the simple algorithm's frequencies identification, it can be seen in Fig. 4.5 that for the choosing of controller coefficients $G(s)=1, K_{1 i j}=0$ and $K_{2 i j}=1$ (simple tuning function), although the estimated frequency almost has the same transient time that we have in the first set fundamental component of proposed method the oscillation looks high. Also, with the second fundamental frequency in Fig. (4.6) it can be seen high oscillation with longer time to converge with the fundamental frequency, more than $4 \mathrm{~s}$ ( approximately two times more than the second set of proposed algorithm), before entering a steady state with fundamental frequency. It should be noted that the frequency adaption gains of the simple tuning function were chosen two times larger than the proposed algorithm as $K a=-4$ or with frequency $20 \%$ to $22 \%$ of the fundamental frequencies one. Thus, we have shown good frequencies identification and low oscillations with the proposed algorithm compared with the simple approach results. 


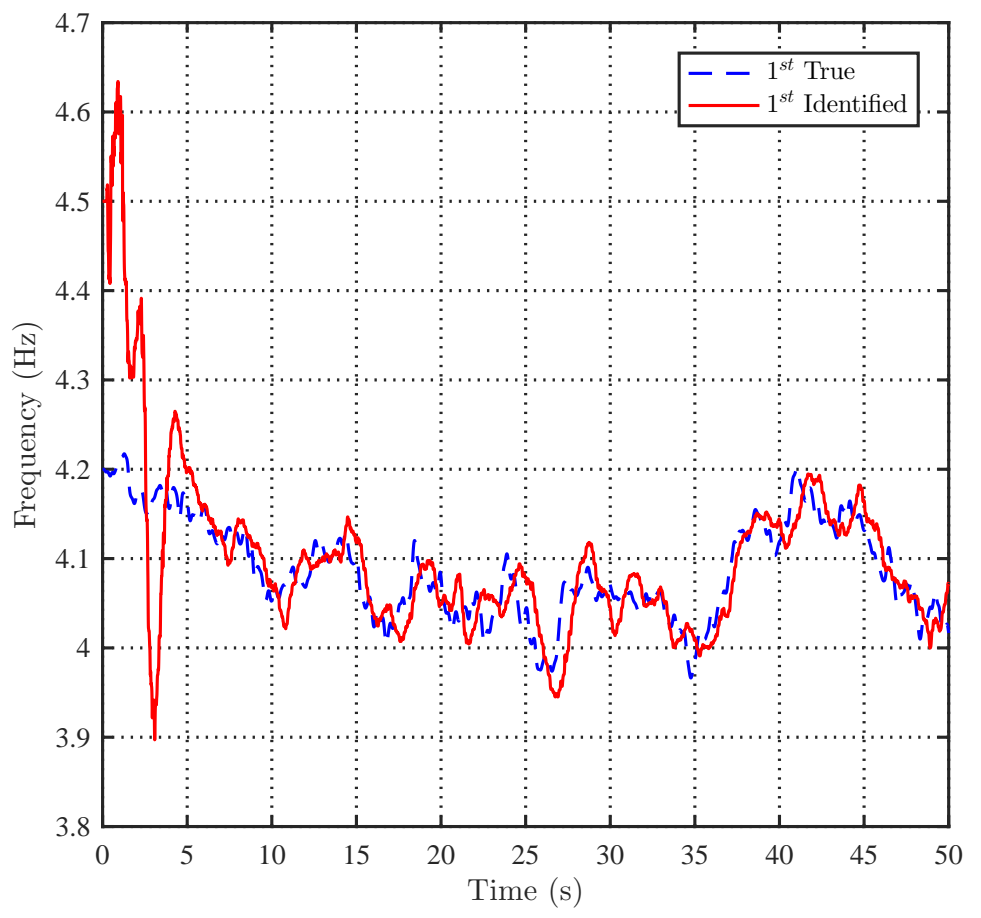

Figure 4.3: Frequency identification for first fundamental component of proposed tuning function.

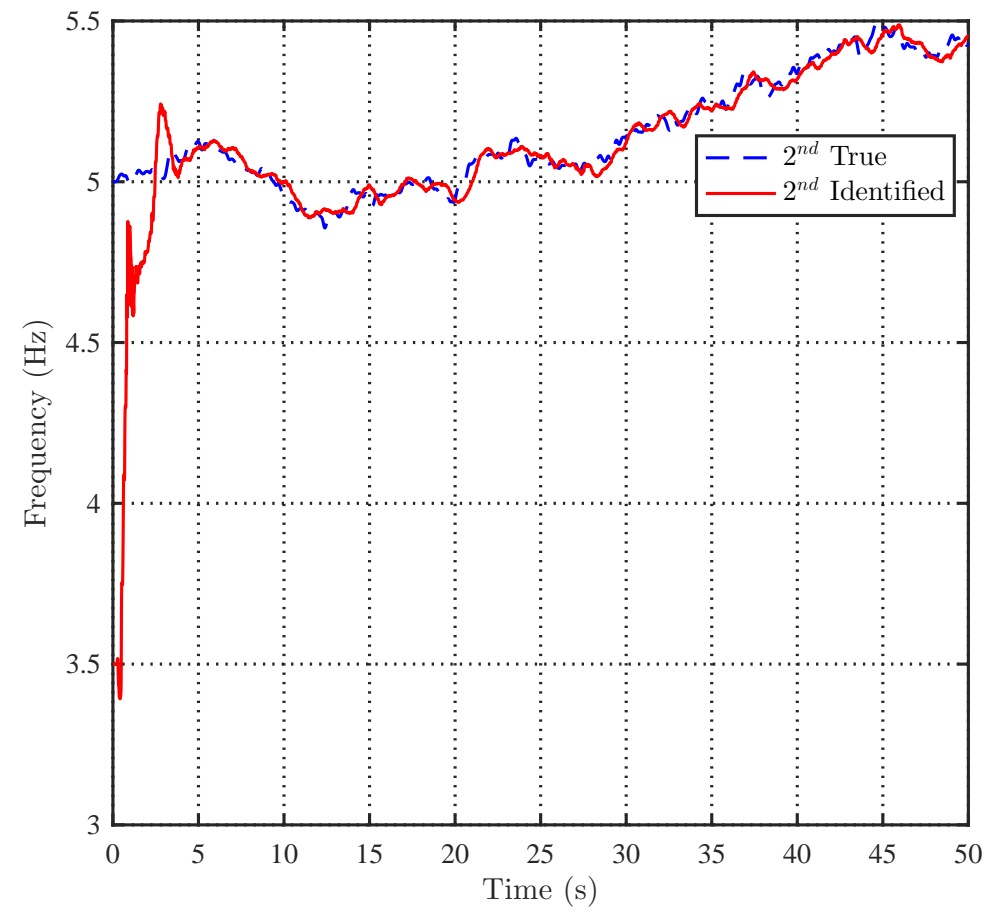

Figure 4.4: Frequency identification for second fundamental component of proposed tuning function. 


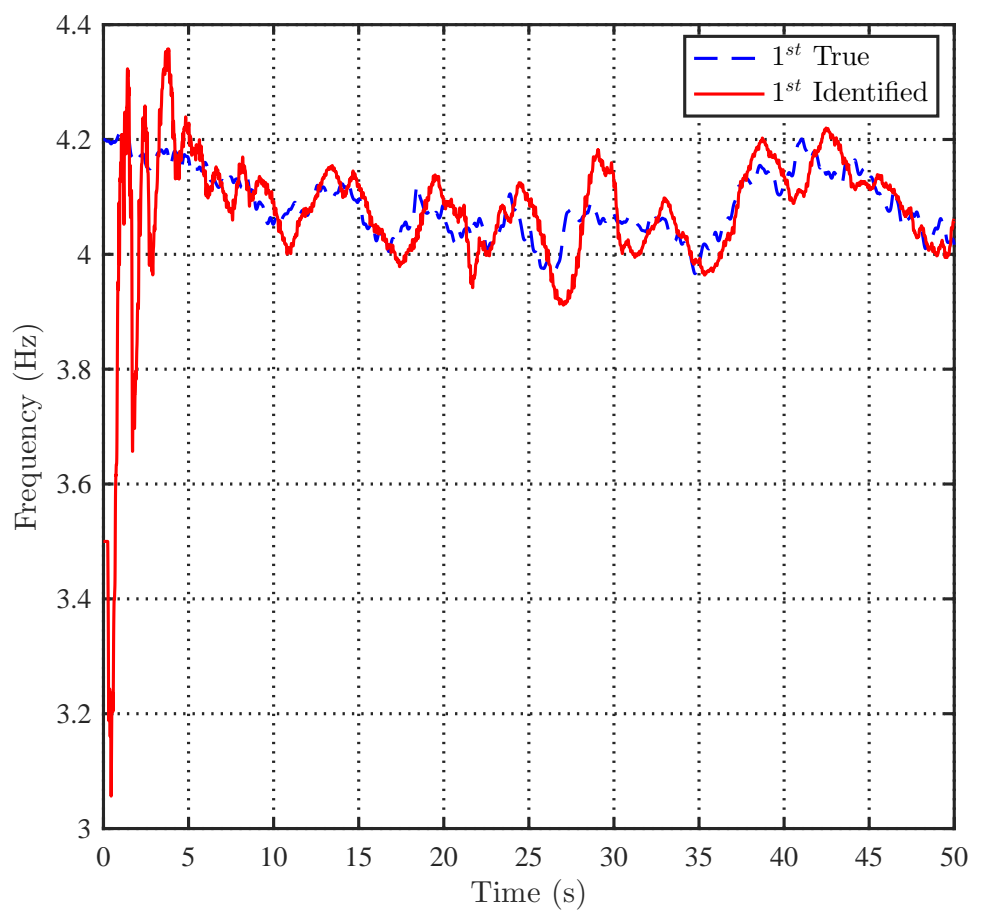

Figure 4.5: Frequency identification for first internal model of simple tuning function.

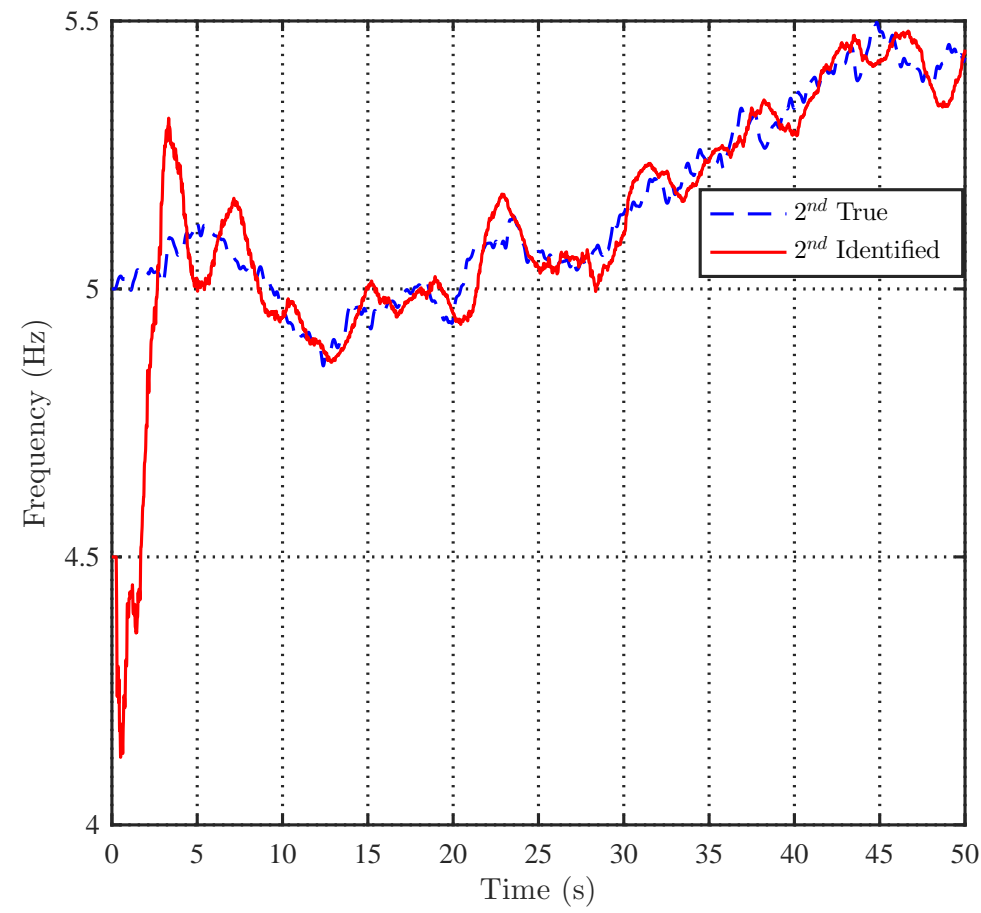

Figure 4.6: Frequency identification for second internal model of simple tuning function 
Now, to get an overview of the signal frequency content and the accuracy of the identified models, the FFT of the signal to be identified and the error signal of the proposed tuning function, simple algorithm $G(s)=1$ and the alternative approach are shown below. In figure 4.7 you can see that the fluctuation frequencies have caused the two fundamentals to be smeared across a range of frequencies. In Fig. 4.7 it can be seen that most of harmonics have been identified although there is a huge DC component of the $e(t)$ which is as expected as we did not attempt to identify it. In Fig. (4.8) it can be seen that the simple tuning function behavior with not only a huge DC component but also a significantly larger error at all of the other frequencies of significance

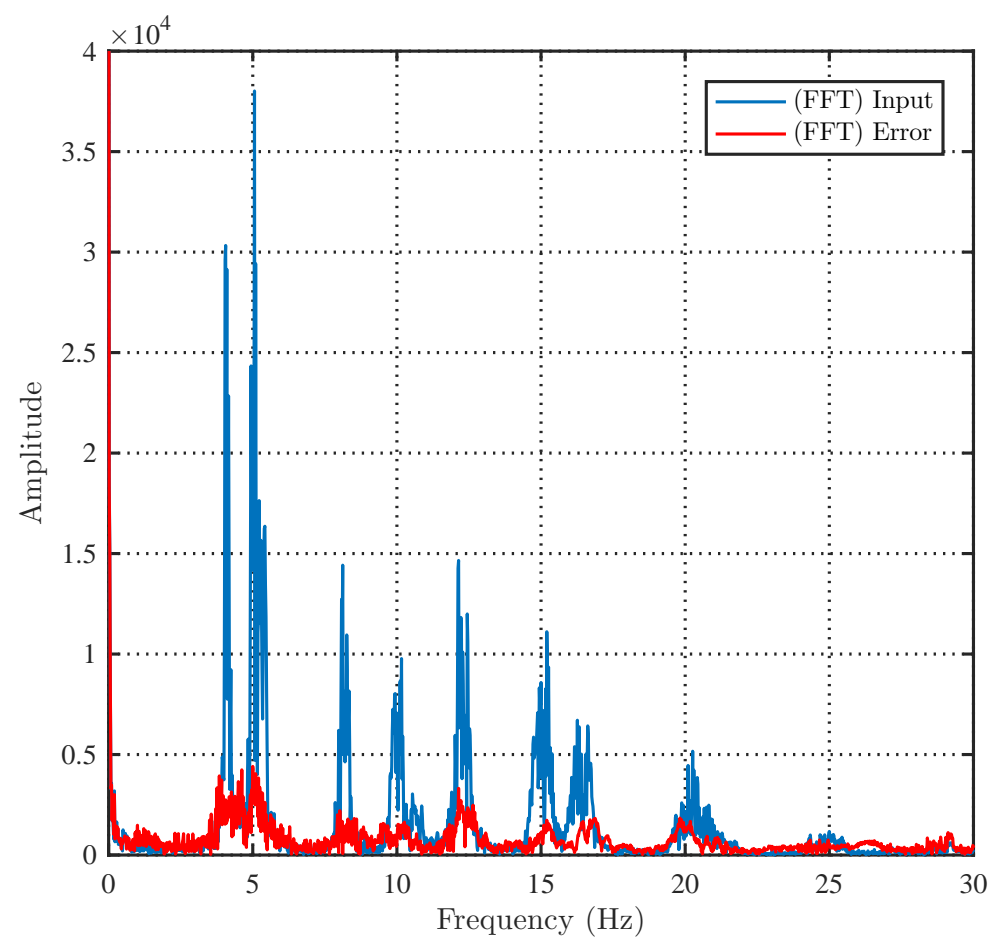

Figure 4.7: Fast Fourier transform of the input signal and error after applying proposed tuning function 


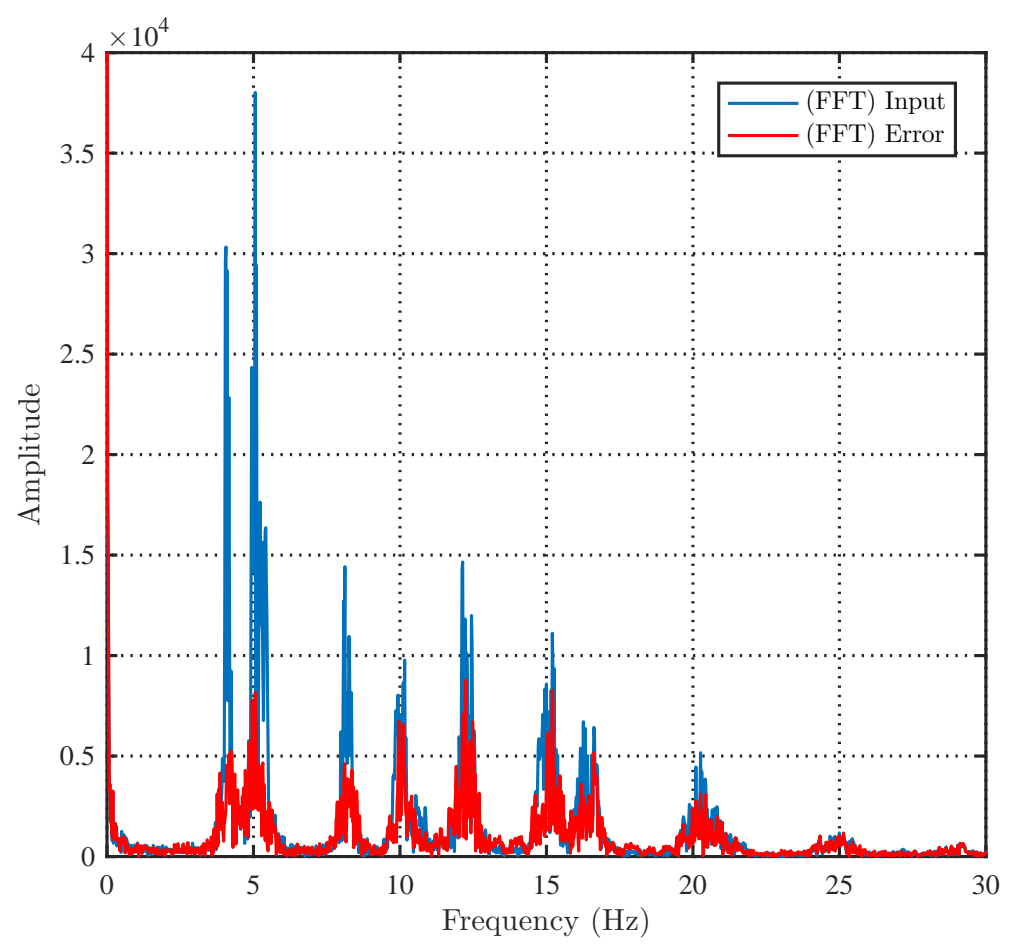

Figure 4.8: Fast Fourier transform of the input signal and error for simple tuning function

As we mentioned previously, we modified our propose algorithm signals tracking by identifying the DC component, and increasing the speed of the dominant poles of the nominal closed loop system. The FFT of the signal to be estimated and the difference of signals of the alternative approach can be seen in figure (4.9), the DC component is identified with very small error due to the much better harmonics identified compared with simple and proposed tuning algorithm. 


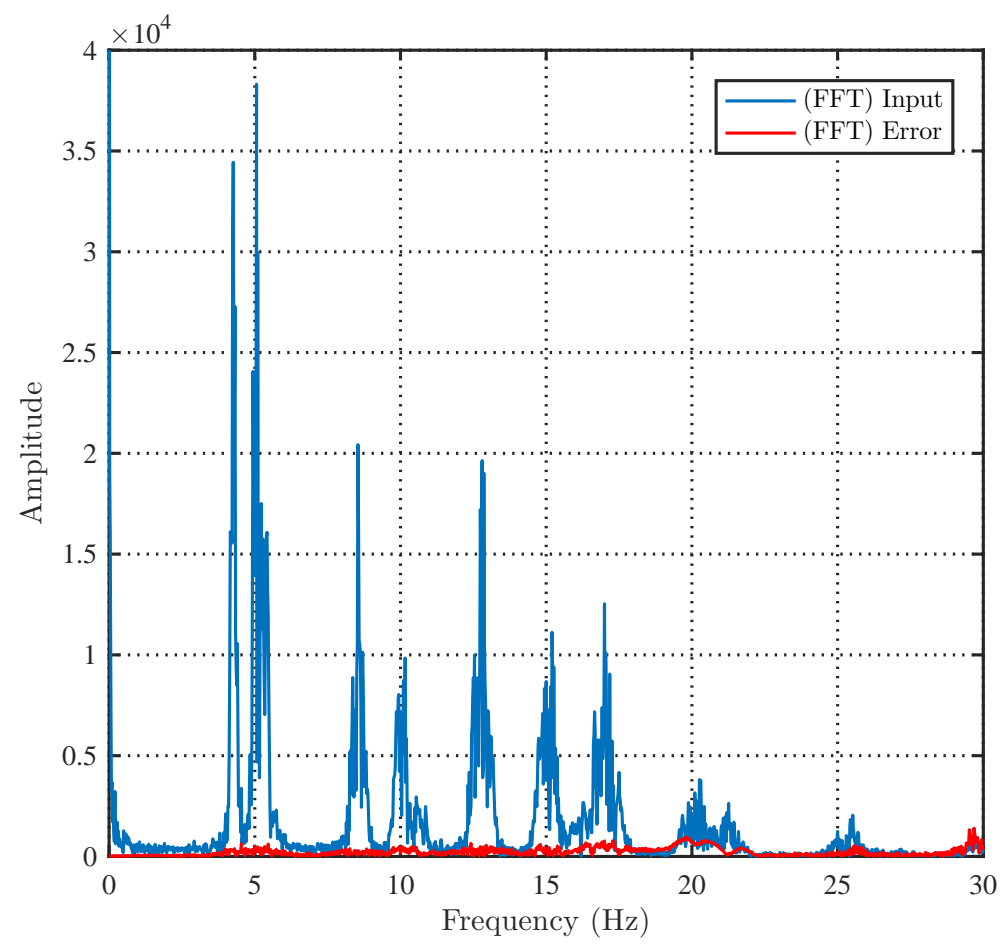

Figure 4.9: Fast Fourier transform of the input signal and error for alternative approach

\subsubsection{Harmonics magnitudes of the synthesized signal}

The harmonics are components or signals with a frequency that is positive integer multiplied by the fundamental frequency [46]. These harmonics, and how many harmonics can be obtained from the periodic disturbances and the magnitude of each harmonic that has been identified for both first and second internal models, can be seen in Fig. 4.10 and 4.11 respectively. Since our reference signal is not a pure sinusoidal, the energy is included in some harmonics beside the fundamental frequency. Therefore, the $1^{\text {st }}$ harmonic is our fundamental frequency, while $2^{\text {nd }}, 3^{\text {rd }}$ and $4^{\text {th }}$ harmonics are multiples of this fundamental frequency. Fig. 4.10 demonstrates how the second harmonic started at low value and then took over the third harmonic at $10 \mathrm{~s}$ before rapidly increasing to take over the first one as well. Unfortunately, with the signal generated by the repetitive controller model, we do not know the true harmonic component of the signal. Since it is driven by a white noise source, we are not surprised or worried by how noisy this estimates look. 


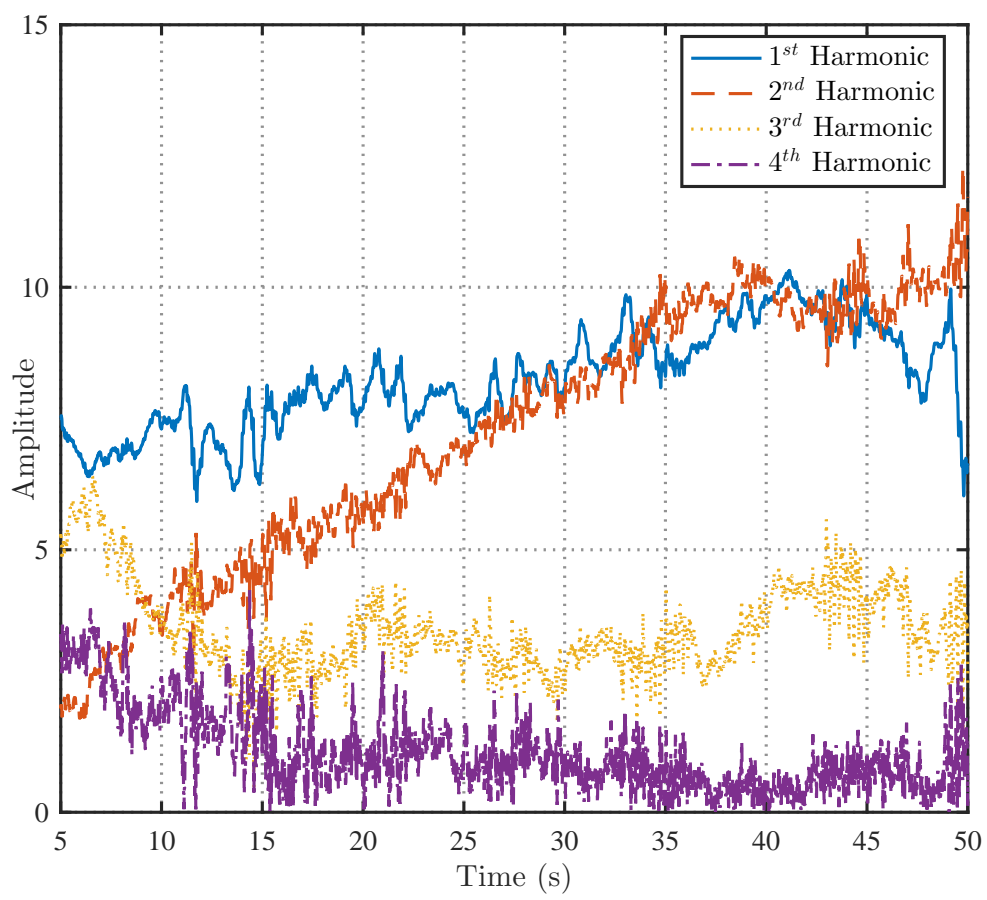

Figure 4.10: Magnitude of each harmonic in the $1^{s t}$ internal model

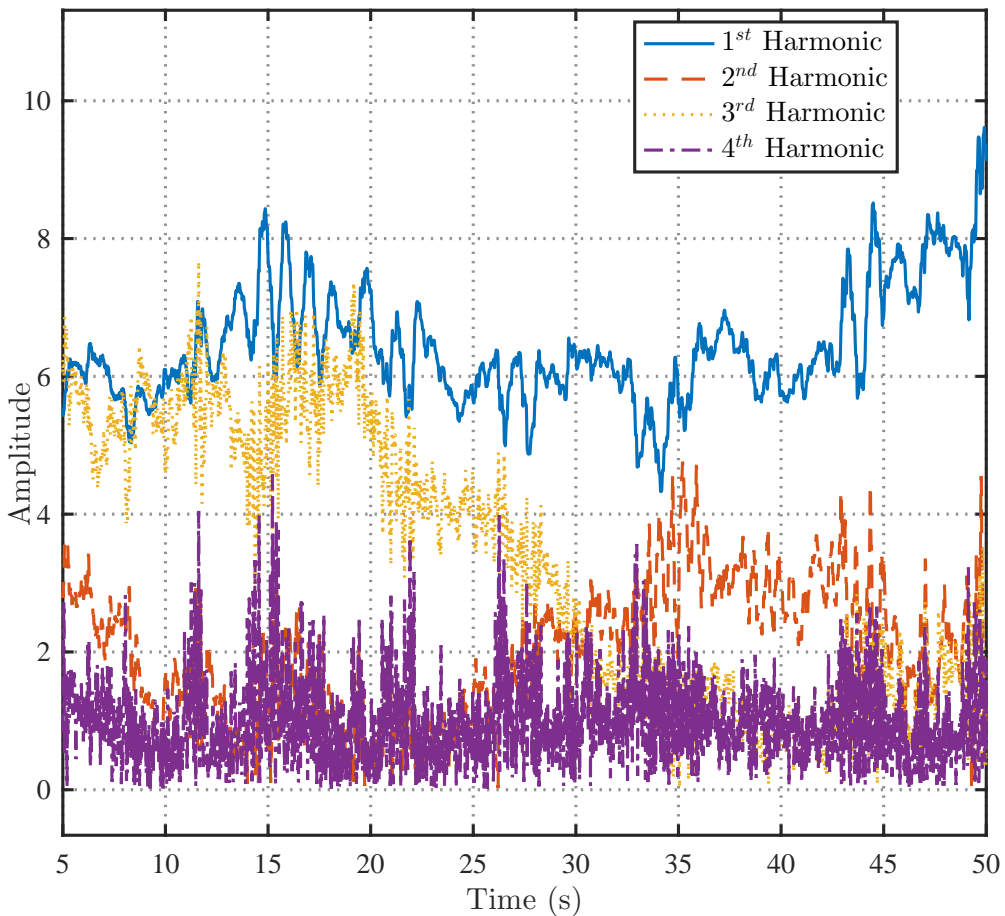

Figure 4.11: Magnitude of each harmonic in the $2^{\text {nd }}$ internal model 


\subsubsection{Comparison of true and identified signal}

In this section, the comparison of a periodic disturbance and identified signal for each internal model is given. In addition, the sum of input $d_{i}(t)$ and output $y_{i}(t)$ signals are compared by the given figures for simple and our proposed tuning function as well as the alternative a approach.

Fig. 4.12 indicates the tracking between the actual periodic disturbance $d_{1}(t)$ of signal generators and the estimated signals of the first set of the internal models of simple, proposed and alternative algorithms. Unlike the model in equation 1.1), this signal had a significant DC component. This was not attempted to be captured in the simple and proposed signal identifiers so there is a significant DC shift in these two signals; however, we have good tracking performance and signal identification of the proposed and the performance of the controller system has been enhanced with alternative algorithm.

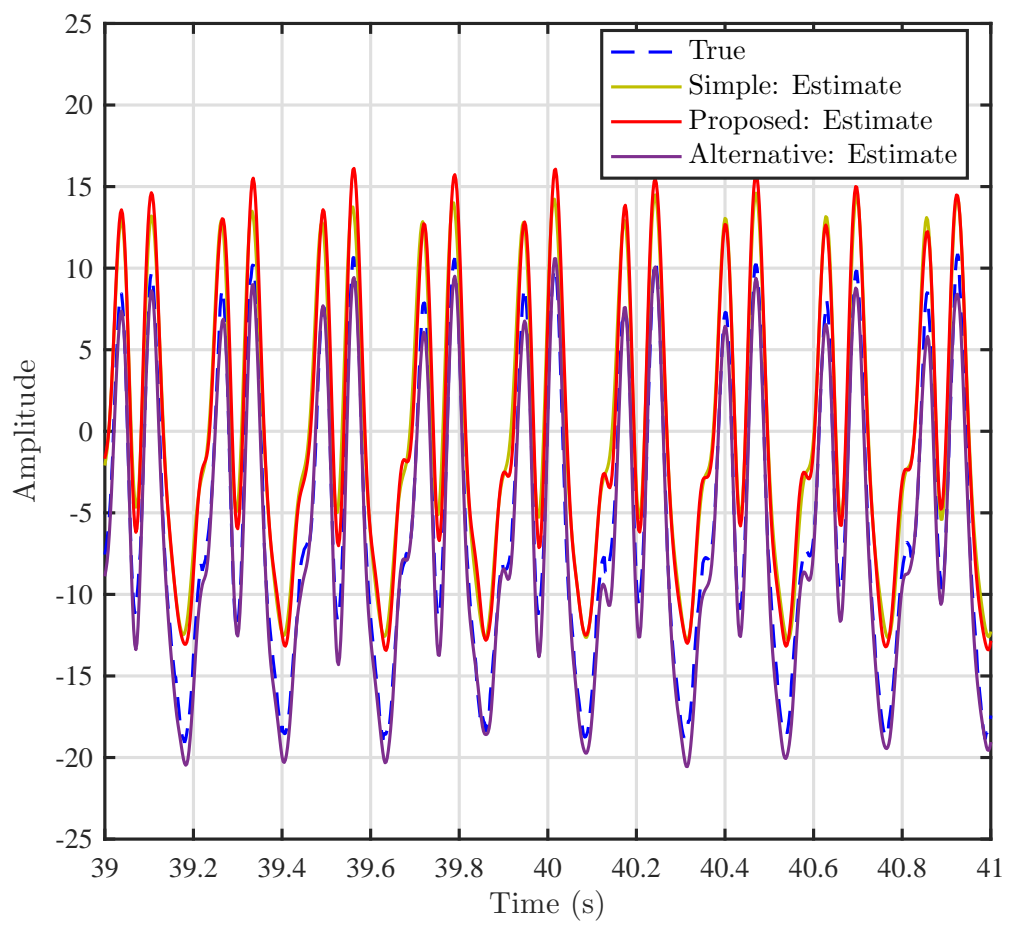

Figure 4.12: Identified versus true signal for the first set of the internal model

Fig. 4.13 show a close up of the actual periodic disturbance $d_{2}(t)$ of signal generators and the identified signal of the second set of the internal models. The alternative algorithm has shown very 
good tracking and periodic signal matches compared with the simple and proposed algorithm due to the significant DC which we have not attempted to identify. In particular, there is no way to distinguish and hence identify the DC content of the two true signals. Again we showed very good amplitude and relative phases matching, and thus we are able to identify these periodic signals in real time.

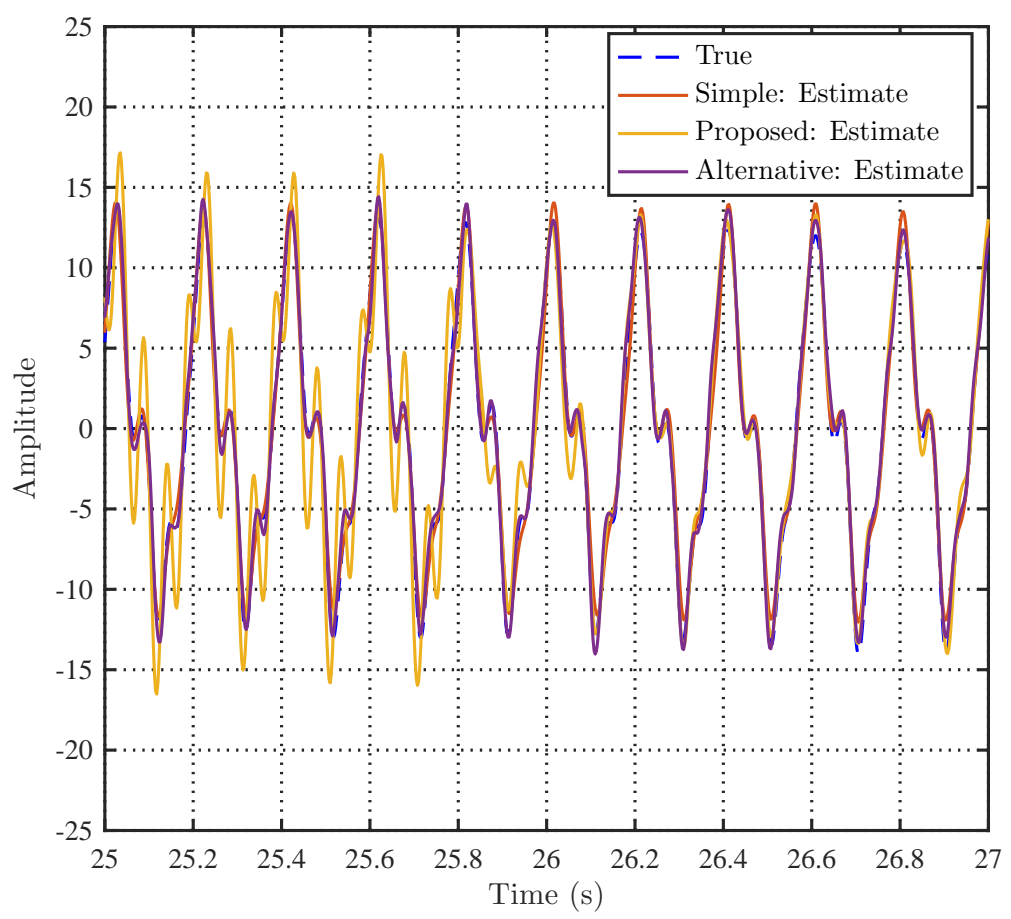

Figure 4.13: Identified versus true signal for the second set of the internal model

Fig. 4.14 displays the quasi-periodic signals $d_{i}(t)$, the identified signal $y(t)$ and their difference $e(t)=1.8550$. After a brief transient we see that e becomes quite small. Note at $42 \mathrm{~s}$ the 5 th harmonic of the 1st signal and the 4th harmonic of the second signal both had frequencies of 21.61 Hz. When a threshold of $0.01 \%$ was chosen for eliminating the redundant internal model, the algorithm went unstable. At the threshold of $0.1 \%$ there was a brief loss $(<0.1 \mathrm{~s})$ of performance in the signal estimation. At a threshold of $0.5 \%$ there was no noticeable loss in quality of the signal estimation. 


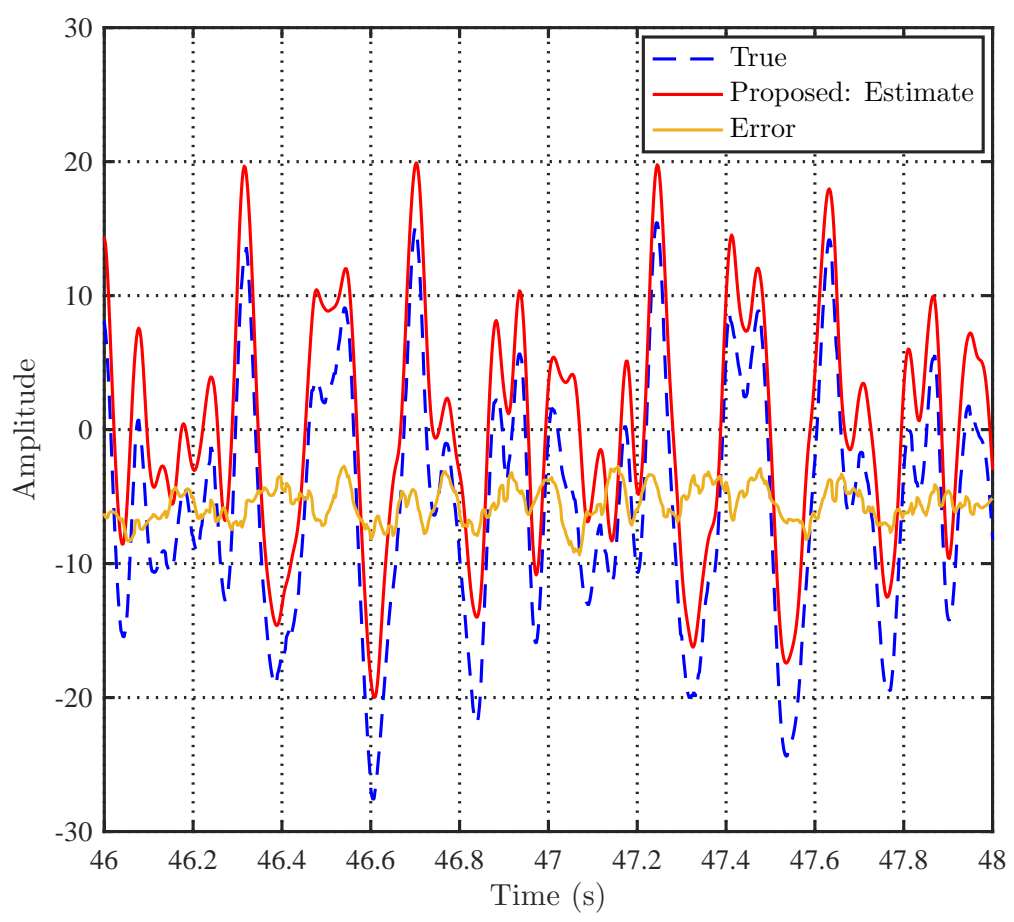

Figure 4.14: Comparison of identified signal, error and real signal with proposed tuning function

As it can be seen in Fig. 4.15 and after eliminating the DC component by subtracting the signal and the error from their means, we can see close up of the actual out put of the signal generators and the identified signal compared with Fig. 4.14 that has significant DC component. Note, at 26 $\mathrm{s}$ the plot shows how fast the input signal started to converge with the identified one. 


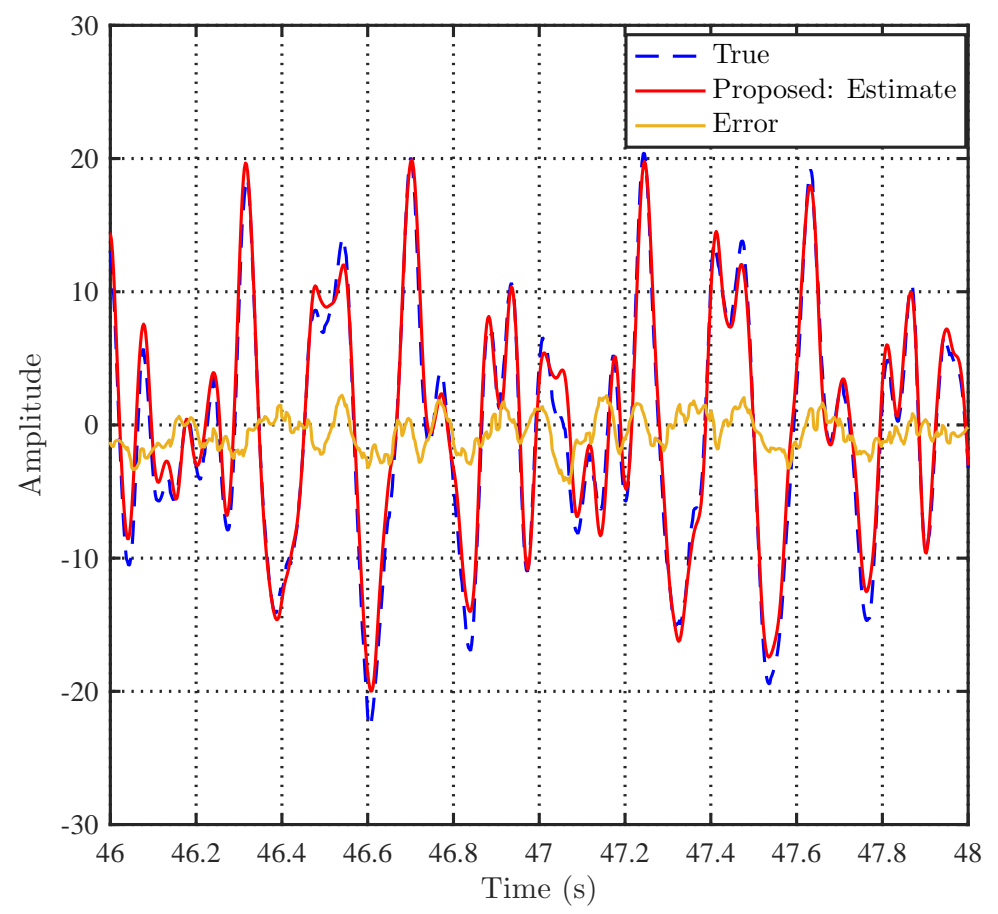

Figure 4.15: Comparison of identified signal, error and real signal without a DC component of proposed tuning function

Fig. 4.16 shows very good signals match, identified and true signal tracking with quiet small error $e(t)=0.6915$. It is much better signal identified due to the rejection of the DC component of the alternative algorithm. 


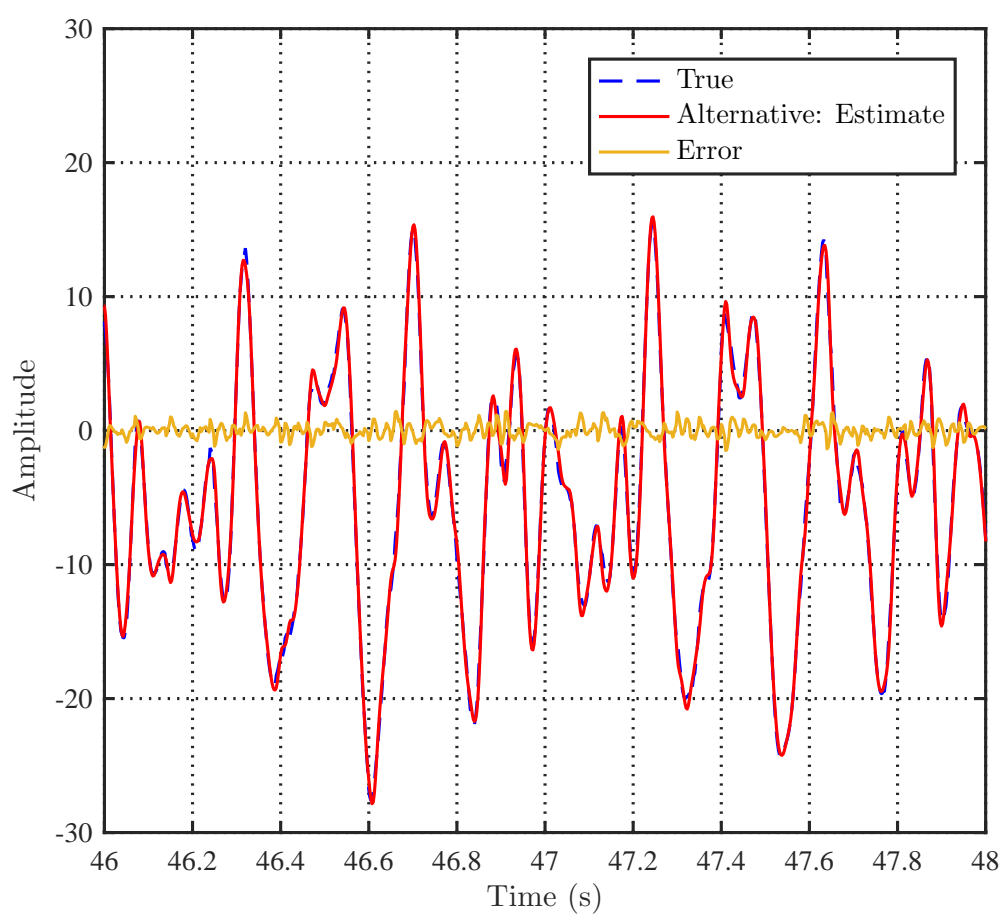

Figure 4.16: Comparison of identified and true signal of alternative proposed algorithm

It can be shown that the choice for controller parameters of $G(s)=1, K_{1 i j}=0$ and $K_{2 i j}=1$ always results in a stable feedback loop for any possible choices of $\hat{\omega}_{i}$. Unfortunately, this leaves the dynamics of the closed loop system uncontrollable and uncertain, and so may require more conservative selections of the adaption gain and increase amplification of measurement noise. Fig. 4.17 demonstrates the low performance of the simply tuned algorithm compared with the proposed and alternative algorithms. The steady state error was about 2.5 and 5 times larger in magnitude of the proposed and alternative algorithm respectively as shown in table 4.4. 


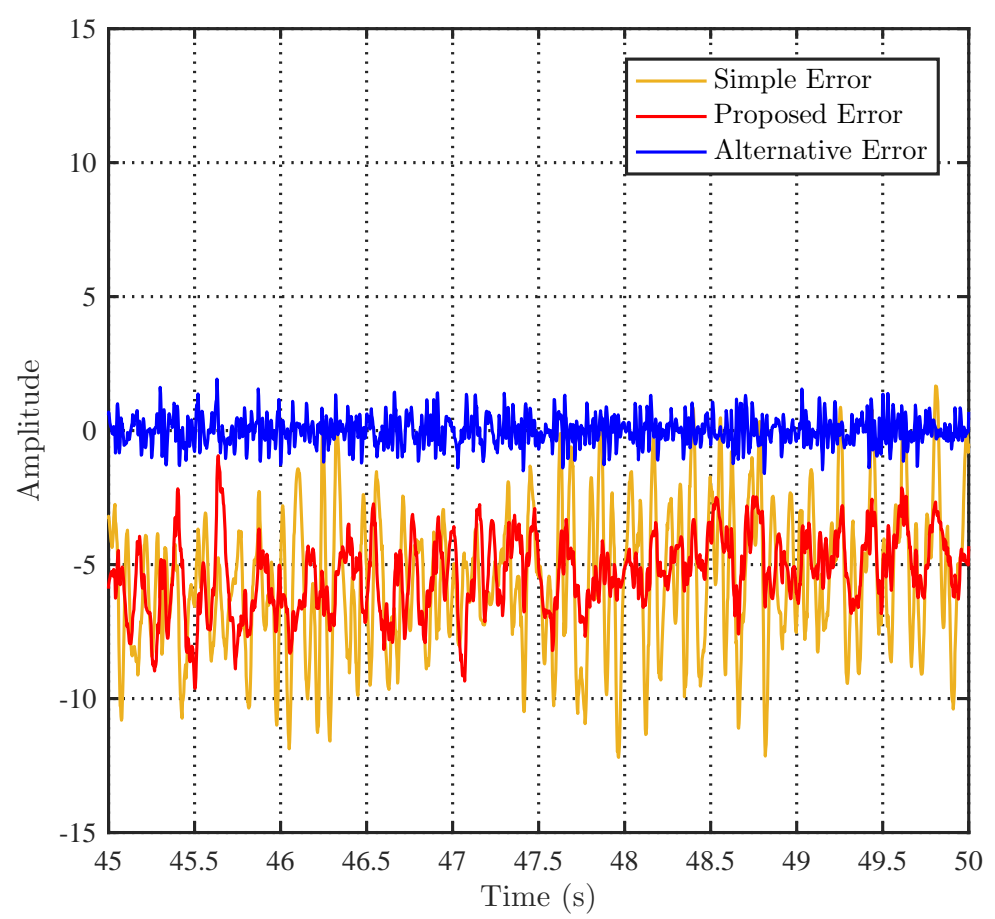

Figure 4.17: Error signals for proposed tuning versus simple tuning and alternative approach

Steady state error

\begin{tabular}{|c|c|c|}
\hline \hline simple approach errore $(t)$ & proposed approach error $e(t)$ & Alternative proposed approach error $e(t)$ \\
\hline 3.7179 & 1.8550 & 0.6915 \\
\hline
\end{tabular}

Table 4.4: Simple, proposed and alternative algorithm error comparison

\subsection{Magnitude identification}

In this particular section we are not only going to identify the fundamental frequencies but also the amplitude of each harmonic of the true signal. $d_{i}(t)$ that is used previously with proposed algorithm has randomly $\bar{A}_{i j}(t)$; in other words, previousely all the harmonics are unknown we will introduce a new signal with known harmonics and relative phases, they are given as 


$$
\begin{aligned}
& \bar{A}_{1,1}(t)=1+.2 * \sin (.05 * t) \\
& \bar{A}_{1,2}(t)=.5+.02 * \cos (.05 * t) \\
& \bar{A}_{1,3}(t)=.3+.01 * \sin (.05 * t) \\
& \bar{A}_{1,4}(t)=.1 * \text { ones }(20001,1) \\
& \bar{A}_{1,5}(t)=.05+.005 * \cos (.05 * t)
\end{aligned}
$$

and for the second set of the true signa

$$
\begin{aligned}
& \bar{A}_{2,1}(t)=1+.5 * \sin (.001 * t) \\
& \bar{A}_{2,2}(t)=.5+.2 * \cos (t) \\
& \bar{A}_{2,3}(t)=.3+.02 * \sin (.05 * t) \\
& \bar{A}_{2,4}(t)=.25 * \text { ones }(20000,0) \\
& \bar{A}_{2,5}(t)=.2+.05 * \cos (.05 * t)
\end{aligned}
$$

where the first and second fundamental frequencies are given as

$$
\begin{aligned}
\omega_{1} & =(4.2+.3 * \sin (.9 * t)) * 2 * p i \\
\omega_{2} & =\left(5+\left[\text { zeros }(5001,1) ;[1: 5000]^{\prime} / 5000 ; \text { ones }(5000,1) ;\right.\right. \\
1 & \left.\left.*[5000:-1: 1]^{\prime} / 5000\right]\right) * 2 * p i
\end{aligned}
$$

Figures. 4.18 and 4.19 show very good tracking and magnitude identification of first and second harmonics of the true signal. 


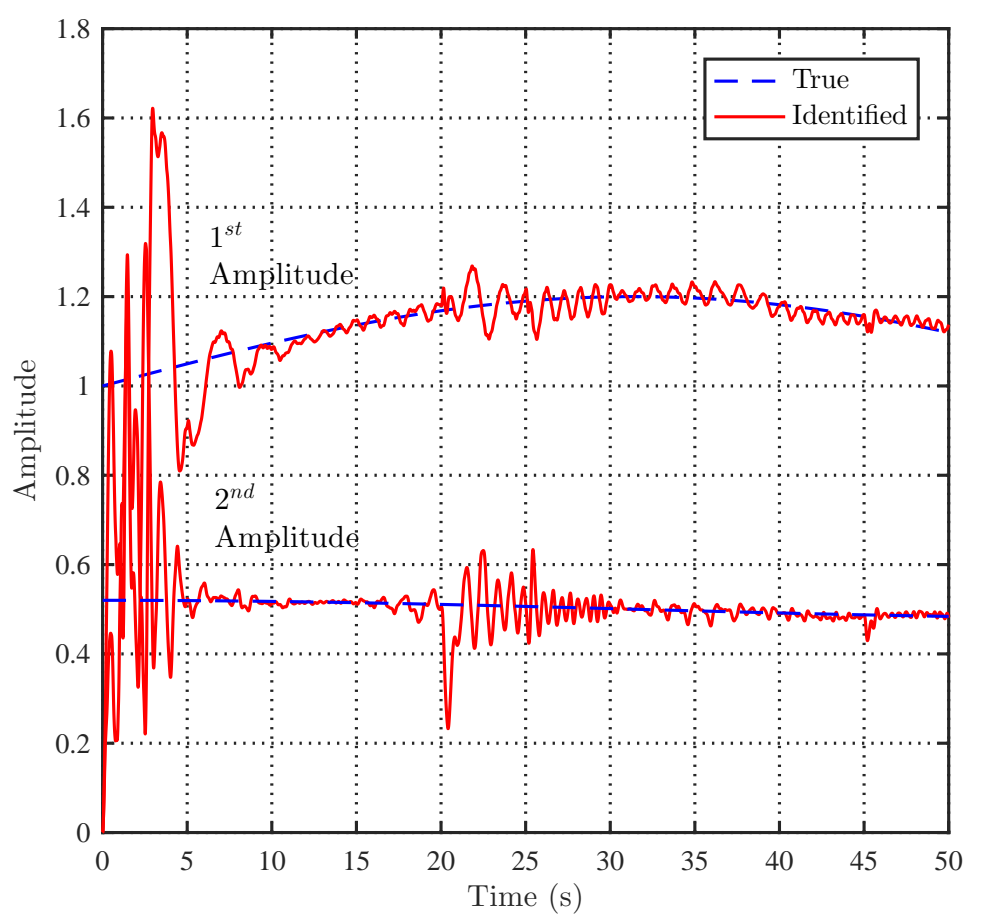

Figure 4.18: Identified amplitudes of first set of the $\mathrm{d}(\mathrm{t})$ harmonic.

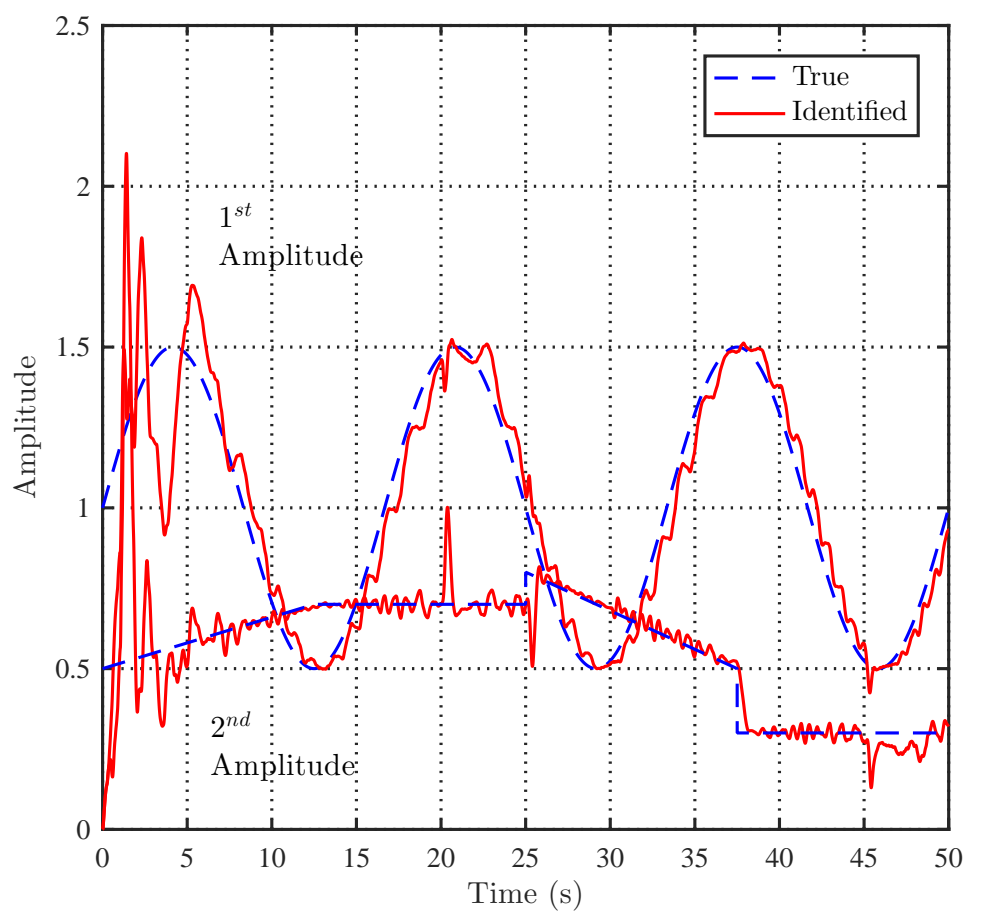

Figure 4.19: Identified amplitudes of second set of the $d(t)$ harmonic. 
Ii can be seen in the figures (4.20) and (4.21) approximately that the modified approach achieved two times faster than the simple and proposed algorithm convergence time. The frequency updated law must be significantly slower than the slowest dynamics of the closed loop system. Since the modified algorithm has much faster dominant poles, it produces better results, in particular better transient characteristics.

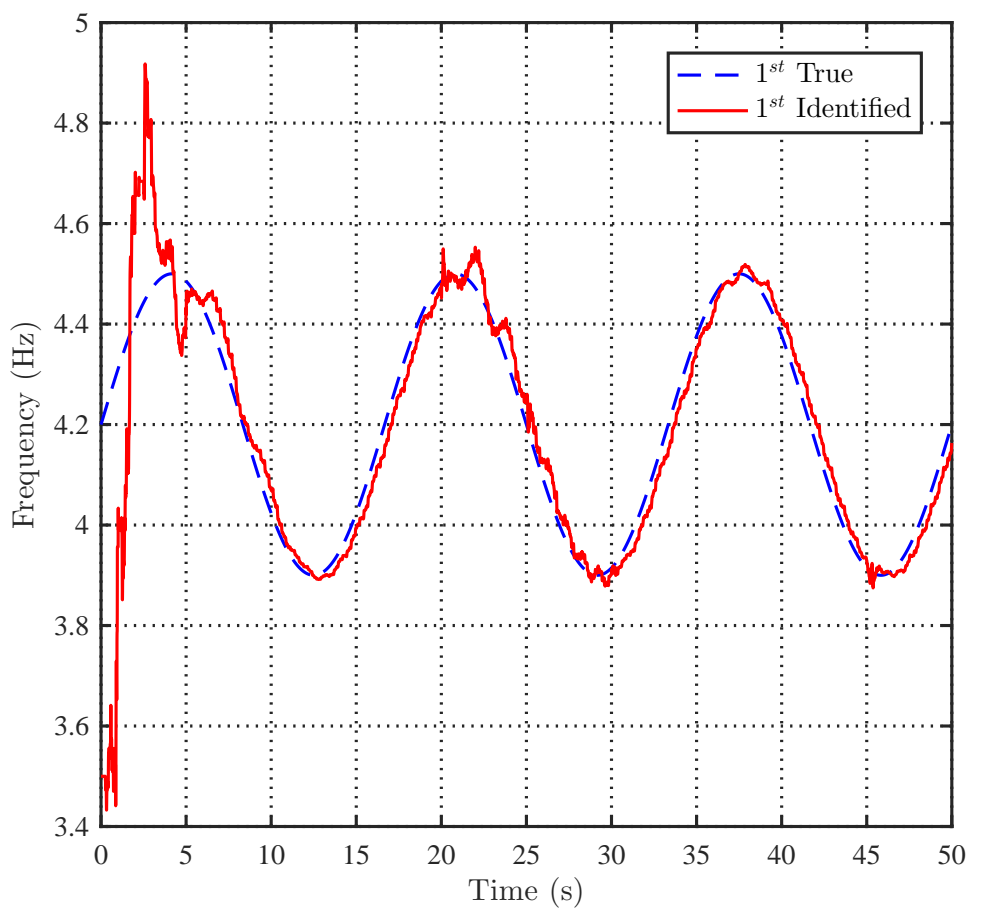

Figure 4.20: Frequency identification for first fundamental component alternative proposed algorithm 


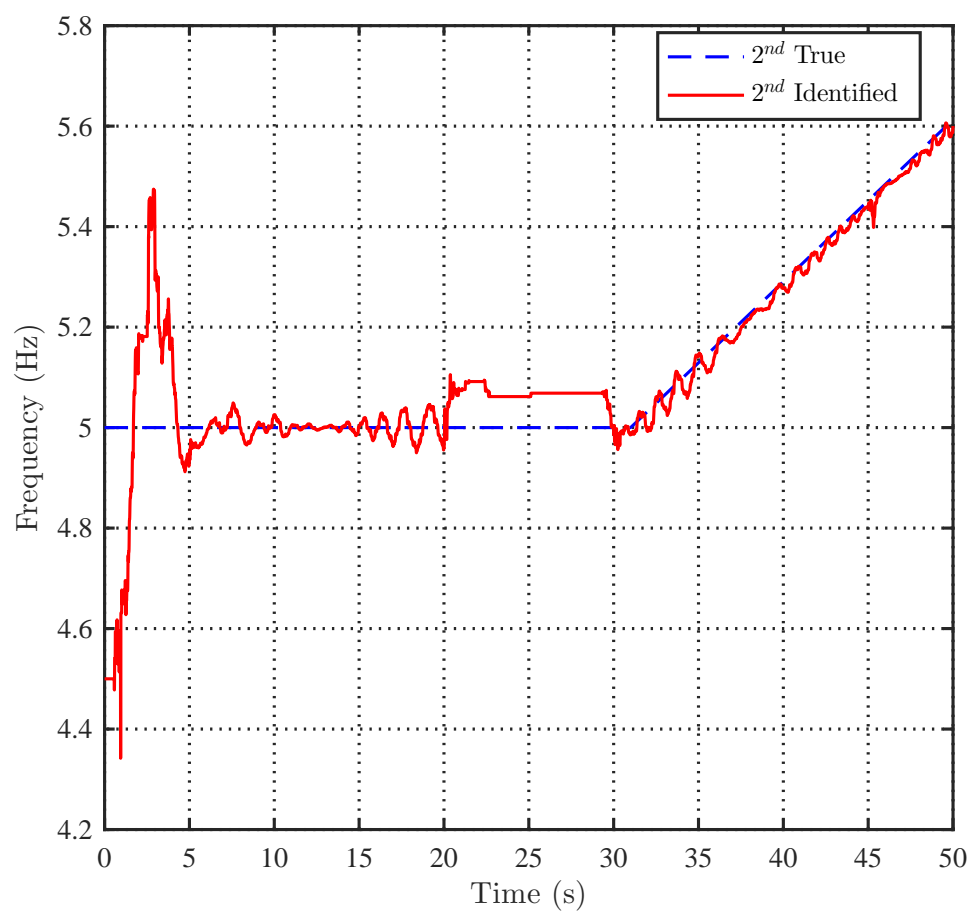

Figure 4.21: Frequency identification for second fundamental component alternative proposed algorithm

\subsubsection{Comparison with HHT algorithm}

In this section, we are going to compare the proposed algorithm with the HHT that we discussed in Sec. 2.3.3.

As we mentioned previously, our algorithm uses the same approximations to estimate the instantaneous frequencies as the HHT. However, this algorithm avoids one of the main restrictions in the HHT, which is the loss of some valuable data with the so-called end effect. Moreover, when we deal with narrowband data, such as in our case, the use of windows become an obstruction, as did the difference between the Hilbert transform and the perfect quadrature of the random function of $\theta(t)$ [32]. Thus, we used an alternative, a notch filter based approach that simultaneously calculates the quadrature signals and decomposes the signal into narrow band signals as shown previously. The main technique with the HHT is EMD, so any given data can be decomposed to a finite number of IMFs. The IMFs produced an instantaneous frequency as a function of time. According to 
the work that has been done in Sec. 2.3.3 and as a result of given equations in (2.10) and (2.14), the IMFs and the instantaneous frequency can be expressed as shown in the figures below.

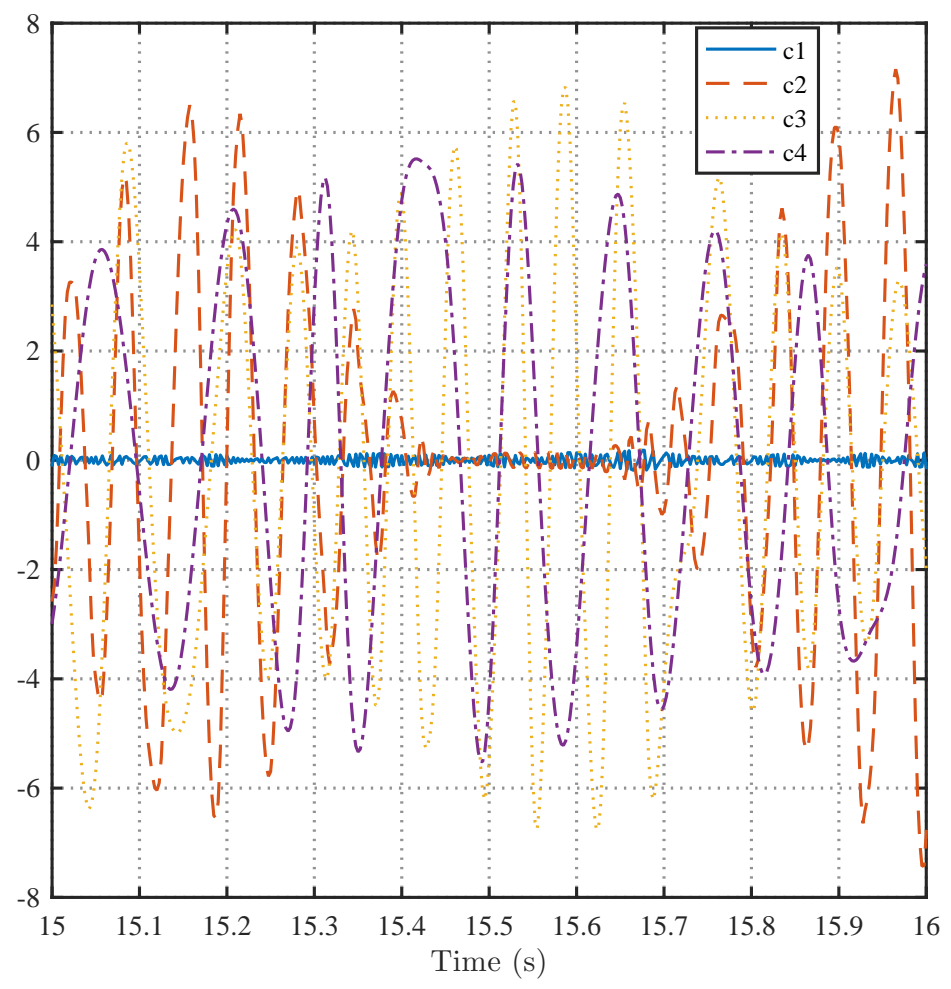

Figure 4.22: IMFs for the first four components

In HHT theory, the lowest number components represent the highest frequency content and should represent the additive white noise and the highest number components should be driven by window edge effects. It is clear from Fig. 4.22 that the first (IMF) is not expressed as a signal in the narrow band such as in the $2^{\text {nd }}, 3^{\text {rd }}$ and $4^{\text {th }}$ intrinsic mode functions. We can also see the end effect of data if we look at Fig. 4.24 to Fig. 4.28, the endpoint of the signal is not the extreme point, so the extreme envelope becomes different at the end, which leads to the significant computational errors in the signal decomposition results [15]. This is considered a main limitation of this algorithm for analyzing non-stationary data. 


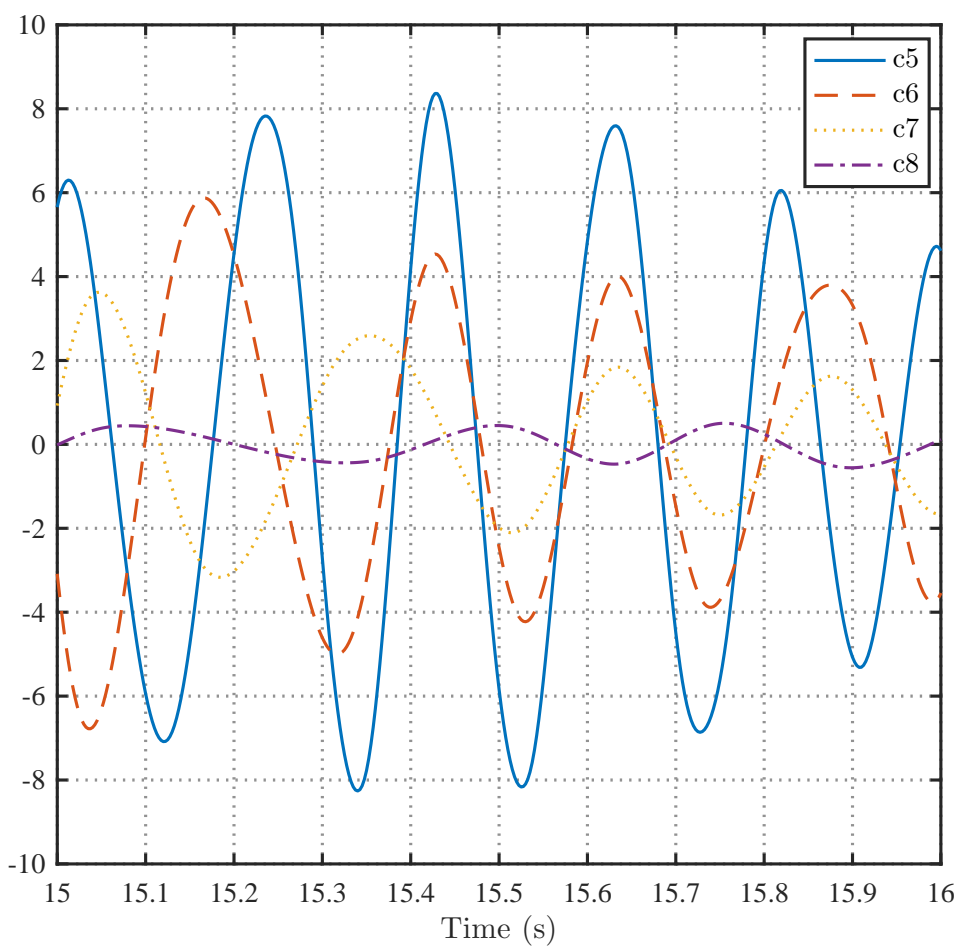

Figure 4.23: IMFs for $5^{\text {th }}, 6^{\text {th }}, 7^{\text {th }}$ and $8^{\text {th }}$ components

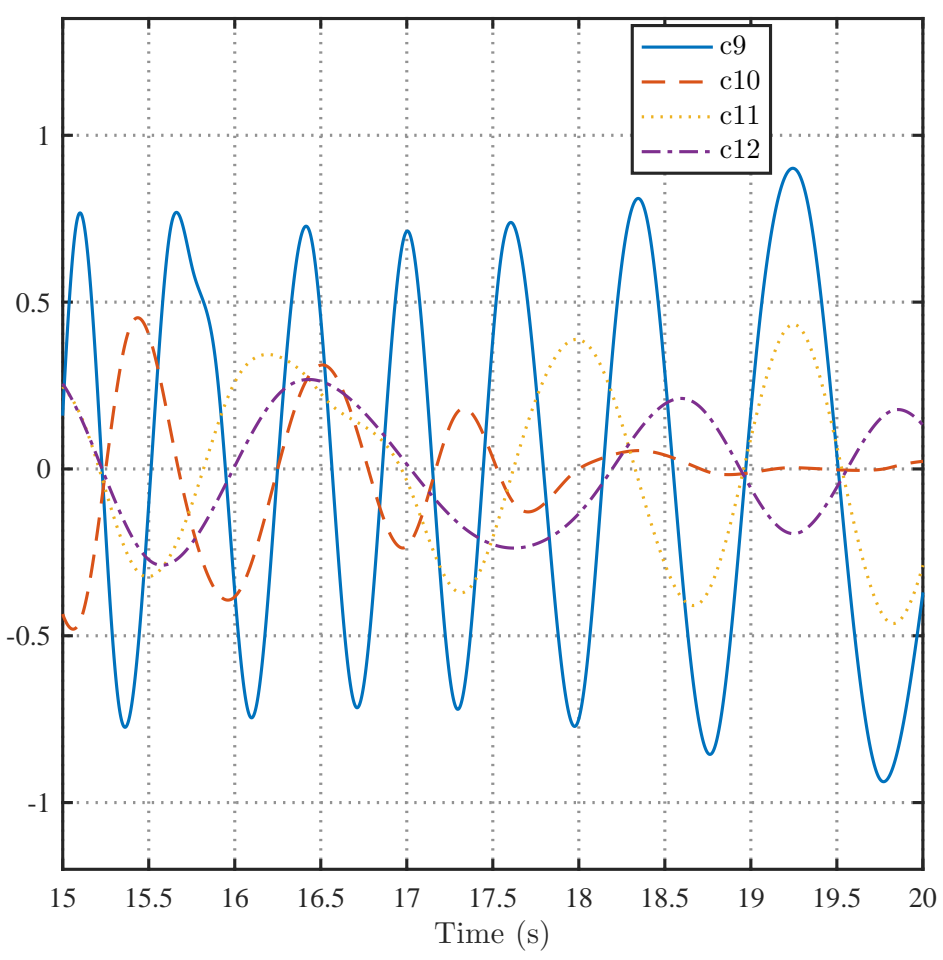

Figure 4.24: IMFs for $9^{\text {th }}, 10^{\text {th }}, 11^{\text {th }}$ and $12^{\text {th }}$ components 
CHAPTER 4. SIMULATION RESULTS AND COMPARISON

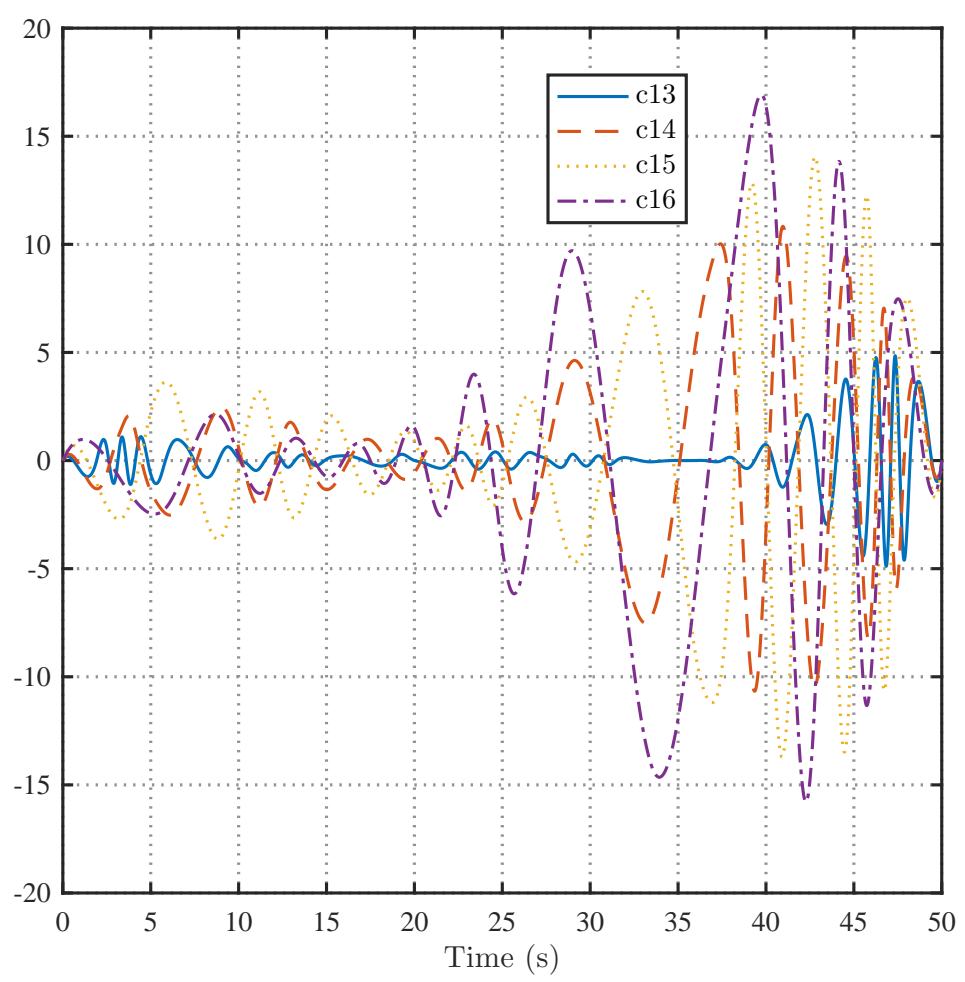

Figure 4.25: IMFs for $13^{\text {th }}, 14^{\text {th }}, 15^{\text {th }}$ and $16^{\text {th }}$ components

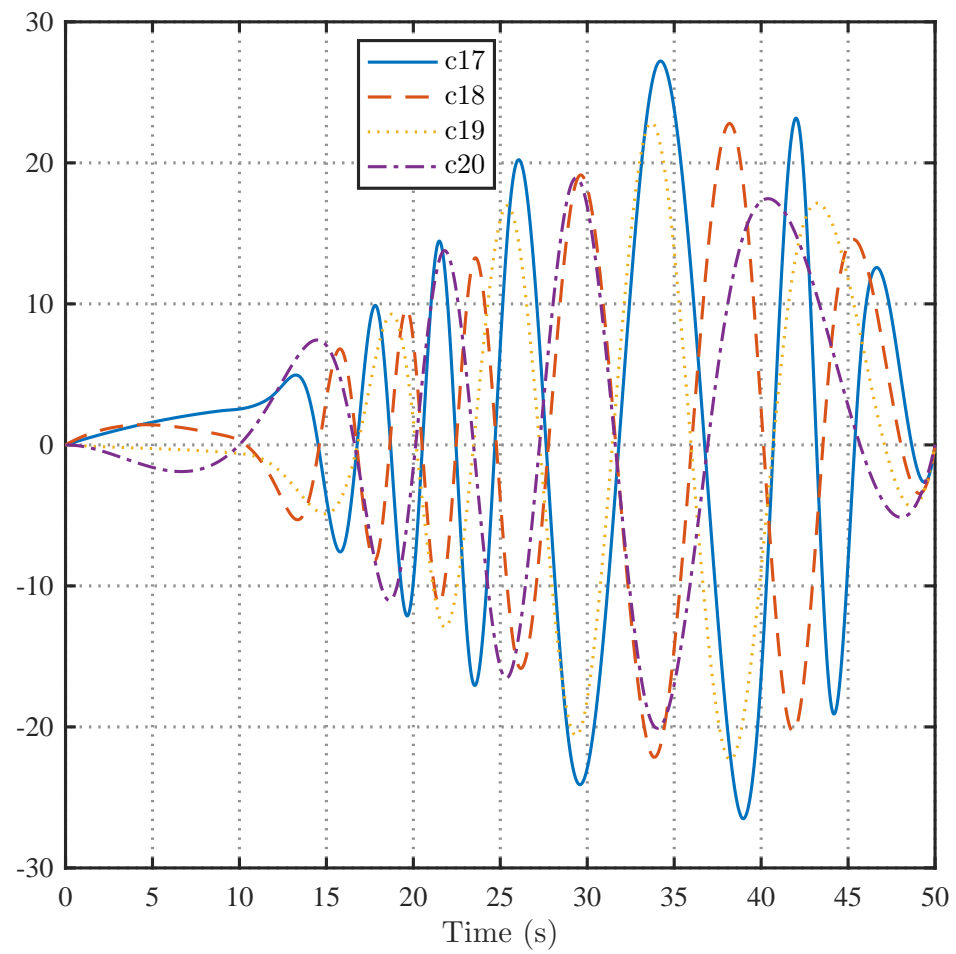

Figure 4.26: IMFs for $17^{\text {th }}, 18^{\text {th }}, 19^{\text {th }}$ and $20^{\text {th }}$ components 
CHAPTER 4. SIMULATION RESULTS AND COMPARISON

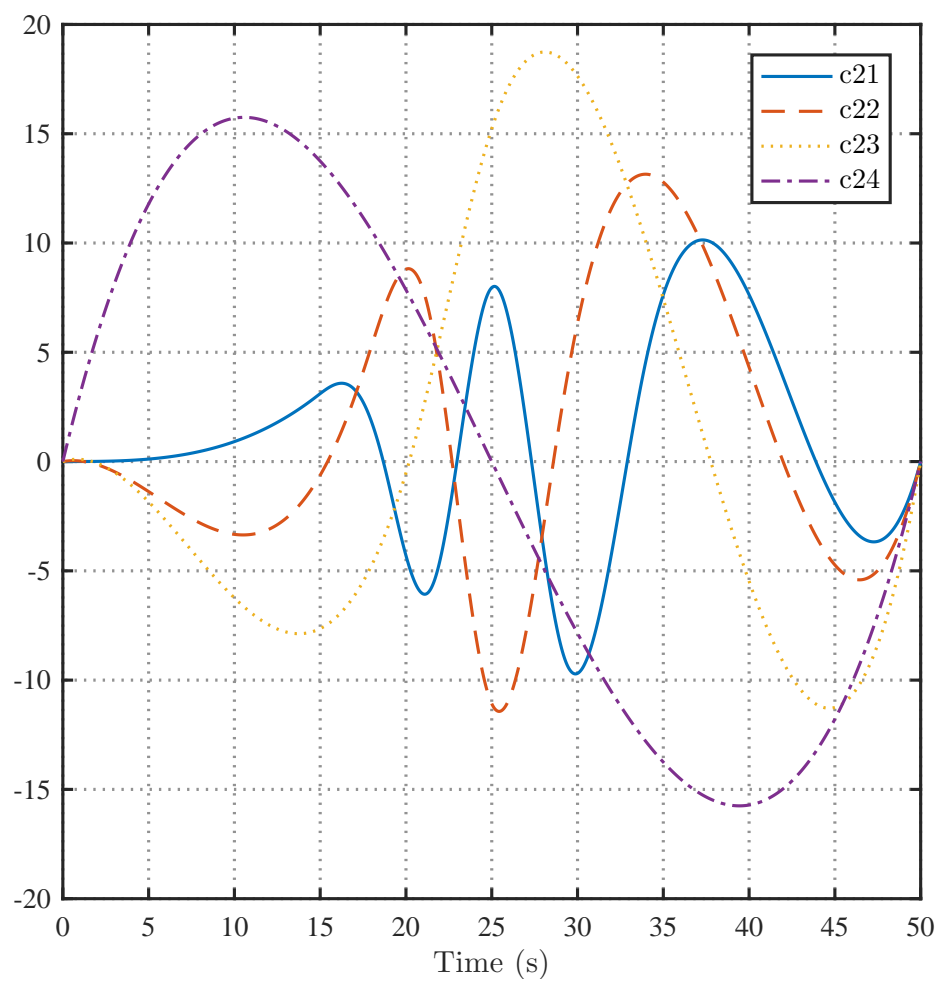

Figure 4.27: IMFs for $21^{s t}, 22^{\text {nd }}, 23^{\text {rd }}$ and $24^{\text {th }}$ components

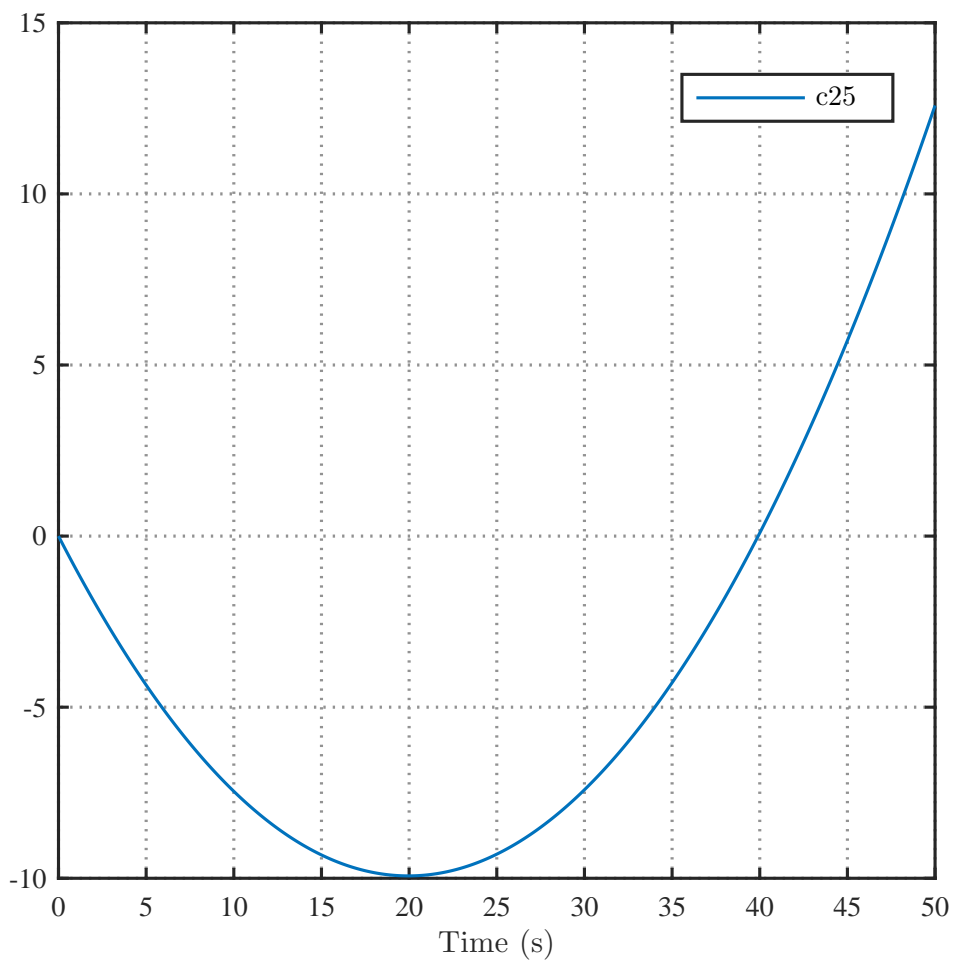

Figure 4.28: IMFs last component 


\subsection{Summary}

In this chapter, we have shown a real time implementation for analyzing non stationary data using developed (IFD). The simulation results of our proposed schema are pointed out as well as a comparison with previous work that has been done with simple approach, $G(s)=1$ is chosen to be one (offline implementation) and (HHT) the algorithm. Moreover, we have demonstrated the high performance of the alternative proposed algorithm not only for frequency identification but also for the DC component and relative phases identification as well as system stability. 


\section{Chapter 5}

\section{Conclusions and Future Directions}

A new tuning approach for an adaptive internal model principle controller is shown in this thesis. First, this thesis has shown previous work ( offline implementation), and how we calculated the feedback transfer function coefficients, these coefficients were calculated by solving a large set of coupled linear equations. The main contribution of this thesis to develop a less computationally approach for computing the feedback controller parameters to meet real time implementation. In other words, a means of tuning an adaptive internal model principle based signal identification algorithm whose computational costs are low enough to allow a realtime implementation is presented in this thesis. The algorithm allows an instantaneous Fourier decomposition of nonstationary signals that have a strongly predictable component, it is shown good fundamental frequencies identification as well as good tracking of amplitude. Also, we proposed an alternative approach to improve system controller performance. Comparison between the proposed algorithm with HHT and an alternative approach was shown with simulation results under Matlab (R2016) environment with solver ode5 (Dormand-prince) selection. Thus, The steady state error was about 2.5 and 5 times larger in magnitude of the proposed and alternative algorithm respectively as shown in table 4.4 . 


\subsection{Conclusion}

This thesis has shown the instantaneous Fourier decomposition algorithm that is based on the orthogonal state variables of an internal model principle controller. Since the main goal of the internal model principle controller is to provide information of the frequency that we need to identify. Thus, the algorithm is locally exponentially stable for identifying these signals. First we examined how we implemented this algorithm off-line by matching the coefficients of numerators and denominators in both equations (3.18) and (3.19). Unfortunately we get a set of $2 n_{t}+4$ coupled equations with $2 n_{t}+4$ unknowns where $n_{t}=\sum_{i=1}^{n} m_{i}$, which is possible to solve off-line and/or theoretical but not practicable to solve in real time. Second, and the main contribution in this thesis is to develop a means of calculating the controller parameters that has a lower computational burden such that it can be successfully implemented in realtime. This thesis shows how the equations can be decoupled into pairs of linear equations which have explicit solutions to met a real time requirement as well as implementing the algorithm with out needing to solve a set or $2 n_{t}+4$ linear equations. As a result of our work in chapter 3 , the algorithm has been successfully implemented online after solving for the issue of overlapping harmonics from different signal components. One of the models is removed from the design process eliminating the dependent equations. The associated controller gains are distributed equally in the implemented controller. Thus, the instantaneous Fourier decomposition is implemented in real time. We applied this developed algorithm in case when we had two fundamental frequencies and as a result, the simulation indicates the validity of our algorithm and the ability to identify the frequencies (very good tracking of time varying frequency) as well as the magnitude and relative phases with uncertain periodic disturbances with good convergence time speed. In other word, in our proposed algorithm we do need to know the structure of the signals. However, we do not have to have accurate estimation of the frequencies in order to be able to design stable feedback loop in advance.Finally, we proposed an alternative approach that has the ability to identify the DC component beside the signals harmonics in order to enhance the system controller performance, and successfully we had much better results frequencies identified and very good signals match as well as the system stability is guaranteed. 


\section{CHAPTER 5. CONCLUSIONS AND FUTURE DIRECTIONS}

\subsection{Future work}

We have seen that the proposed and alternative algorithms have successfully implemented in realtime with very good performance of signals tracking and frequencies identifying with high speed of convergence time, some works are still to be work on. Our future work will be conducted to identify the uncertain frequencies of periodic signals and eliminate periodic disturbances in discrete state space form, so the Matlab simulation can be performed much faster.. 


\section{Bibliography}

[1] L. Sujbert A. R. V. Koczy, G. Simon and M. Fek. "A fast filter-bank for adaptive fourier analysis,". In IMTC/98 Conference Proceedings. IEEE Instrumentation and Measurement Technology Conference. Where Instrumentation is Going (Cat. No.98CH36222), vol. 2, pages 915-918, 1998.

[2] A. N. Akansu and A. R. Haddad. "Multiresolution Signal Decomposition: Transforms, Subbands, and Wavelets,". Academic pr inc, 2000.

[3] M. Bodson and S. C. Dougla. "Adaptive algorithms for the rejection of sinusoidal disturbances with unknown frequency,". In Automatica, vol. 33, no. 12, pages 2213-2221, 1997.

[4] M. Bodson, A. Sacks, and P. Khosla. "Harmonic generation in adaptive feedforward cancellation schemes,". In Decision and Control, 1992., Proceedings of the 31st IEEE Conference on, vol. 2, pages 1261-1266, 1992.

[5] P. Bremaud. "Mathematical Principles of Signal Processing,". Springer, 2002.

[6] L. J. Brown and Q. Zhang. "Control for canceling periodic disturbances with uncertain frequency,". In Decision and Control, 2001. Proceedings of the 40th IEEE Conference on, vol. 5, pages 4909-4914, 2001.

[7] L. J. Brown and Q. Zhang. "Identification of periodic signals with uncertain frequency,". In IEEE Transactions on Signal Processing, vol. 51, no. 6, pages 1538-1545, 2003. 
[8] L. J. Brown and Q. Zhang. "Periodic disturbance cancellation with uncertain frequenc,". Automatica, vol. 40, pages 631-637, 42004.

[9] M. Tomizuka C. Kempf, W. C. Messner and R. Horowitz. "Comparison of four discrete-time repetitive control algorithms,". In IEEE Control Systems, vol. 13, no. 6, pages 48-54, 1993.

[10] S. Riemenschneider C. Qiuhui, N. Huang and Y. Xu. "A b spline approach for empirical mode decompositions,". Advances in Computational Mathematics, vol. 24, no. 1, pages 171-195, 2006.

[11] J. O. Chapa and R. M. Rao. "Algorithms for designing wavelets to match a specified signal,". In IEEE Transactions on Signal Processing, vol. 48, no. 12, pages 3395-3406, 2000.

[12] I. Daubechies. "The wavelet transform, time-frequency localization and signal analysis,". In IEEE Transactions on Information Theory, vol. 36, no. 5, pages 961-1005.

[13] D. Donnelly. "The fast fourier and hilbert-huang transforms: a comparison,". In The Proceedings of the Multiconference on "Computational Engineering in Systems Applications, vol. 1, pages 84-88, 2006.

[14] B. A. Francist and W. M. Wonham. "The internal model principle of control theory,". pages $457-465,1976$.

[15] X. L. Li G. Yang X. B. Sun M. X. Zhang and XR. Liu. "Study on ways to restrain end effect of hilbert-huang transform,". Journal of Computer, vol. 25, no.3, pages 22-31, 2014.

[16] D. Gabor. "Theory of communication. part 1: The analysis of information,". Journal of the Institution of Electrical Engineers-Part III: Radio and Communication Engineering, vol. 93, no. 26, pages 429-457, 1945.

[17] H. Shim H. Kim and N. H. Jo. "Adaptive add-on output regulator for rejection of sinusoidal disturbances and application to optical disc drives,". In IEEE Transactions on Industrial Electronics, vol. 61, no. 10, pages 5490-5499, Oct 2014. 
[18] C. Du H. Li and Y. Wang. "A novel algorithm for unknown periodic disturbance cancellation in hdds,". In 2008 10th International Conference on Control, Automation, Robotics and Vision, pages 97-102, 122008.

[19] S. O. Haykin. "Adaptive Filter Theory,", volume vol. 2. Prentice Hall, 4 edition, 2001.

[20] N. E. Huang. "Introduction to the hilbert huang transform and its related mathematical problems,". pages 1-26, 2003.

[21] M. N. Lee J. H. Moon and M. J. Chung. "Repetitive control for the track-following servo system of an optical disk drive,". In IEEE Transactions on Control Systems Technology, vol. 6, no. 5, pages 663-670, 1998.

[22] E. Mohsen L. J. Brown and J. Chen. "A real time alternative to the hilbert huang transform based on internal model principle,". J Electr Electron, (Syst 6: 233), July 2017.

[23] Y. C. Lim and B. F. Boroujeny. "Fast filter bank (ffb),". In IEEE Transactions on Circuits and Systems II: Analog and Digital Signal Processing, vol. 39, no. 5, pages 316-318, 1992.

[24] L. Ljung. "System identification,". prentice hall information and system sciences. Prentice hall PTR, upper saddle river,NJ 07458, second edition, 1999.

[25] A. Sacks M. Bodson and P. Khosla. "Harmonic generation in adaptive feedforward cancellation schemes,". In IEEE Transactions on Automatic Control, vol. 39, no. 9, pages 1939-1944, 1994.

[26] J. S. Jensen M. Bodson and S. C. Douglas. "Active noise control for periodic disturbances,". In IEEE Transactions on Control Systems Technology, vol. 9(no. 1):200-205, 2001.

[27] K. Usui M. Nagashima and M. Kobayashi. "Rejection of unknown periodic disturbances in magnetic hard disk drives,". In IEEE Transactions on Magnetics, vol. 43, no. 9, pages 3774-3778, 2007. 
[28] Y. Ma. "An Iterative Approach to Automatic Music Transcription and Audio Signal Decomposition,". phdthesis, The University of Western Ontario London, 2009.

[29] N. Malhotra. "Online tip voltage and dynamic resistance measurement in rsw process,", 2005.

[30] R. Marino and P. Tomei. "Disturbance cancellation for linear systems by adaptive internal models,". pages 1494-1500, 52013.

[31] E. Mohsen and L. J. Brown. "Realtime implementation of an internal-model-principle signal identifier,". In 2017 IEEE 30th Canadian Conference on Electrical and Computer Engineering (CCECE), pages 1-5, April 2017.

[32] S. R. Long N. E. Huang M. L. Wu W. Qu and S. P. S. Samuel. "Applications of hilbert-huang transform to non-stationary financial time series analysis,". Applied Stochastic Models in Business and Industry, vol. 19, no. 3, pages 245-268, 2003.

[33] N. C. Yen C. C. Tung N. E. Huang Z. Shen S. R. Long M. C. Wu H. H. Shih Q. Zheng and H. H. Liu. "The empirical mode decomposition and the hilbert spectrum for nonlinear and non-stationary time series analysis,". no. 454, pages 903-995, 1998.

[34] A. Nehorai. "A minimal parameter adaptive notch filter with constrained poles and zeros,". In IEEE Transactions on Acoustics, Speech, and Signal Processing, vol. 33, no. 4, pages 983-996, 1985.

[35] P. A. Regalia. "An improved lattice-based adaptive iir notch filter,". In IEEE Transactions on Signal Processing, vol. 39, no. 9, pages 2124-2128, Sep 1991.

[36] T. Omata S. Hara, Y. Yamamoto and M. Nakano. "Repetitive control system: a new type servo system for periodic exogenous signals,". In IEEE Transactions on Automatic Control, vol. 33, no. 7, pages 659-668, 1988. 
[37] S. Bittanti S. M. Savaresi and H. C. So. "Closed-form unbiased frequency estimation of a noisy sinusoid using notch filters,". In IEEE Transactions on Automatic Control, vol. 48, no. 7, pages 1285-1292, July 2003.

[38] M. Claudio S. S. Radomir and A. Jaakko. "Fourier analysis on finite groups with applications in signal processing and system design,". John wiley \& sons inc, 2005.

[39] A. Salmanpour. "A new signal processing approach to study action potential content in sympathetic neural signals, ". Phdthesis, 42010.

[40] Y. Sun. "Instantaneous fourier series estimation,". Mathesis, 62006.

[41] C. C. Chung W. Kim, H. Kim and M. Tomizuka. "Adaptive output regulation for the rejection of a periodic disturbance with an unknown frequency,". In IEEE Transactions on Control Systems Technology, vol. 19, no. 5, pages 1296-1304, Sept 2011.

[42] L. J. Wei and L. Y. Ching. "Designing the fast filter bank with a minimum complexity criterion,". In Seventh International Symposium on Signal Processing and Its Applications, 2003. Proceedings, vol. 39, pages 279-282, 2003.

[43] B. Widrow and S. D. Steams. "Adaptive signal processing,", volume vol. 15. Prentice-hall Englewood Cliffs, NJ, 1985.

[44] Wikipedia. "Cooley-Tukey fft algorithm," wikipedia, the free encyclopedia, 2017. [Online; accessed 9-March-2017].

[45] Wikipedia. "Hilbert-Huang transform," wikipedia, the free encyclopedia, 2017. [Online; accessed 9-March-2017].

[46] K. W.Kennan and D. Grantham. "The technique of orchestration,". Prentice Hall, sixth edition edition, 2001. 
[47] K. C. Chu Y. Wang and T. C. Tsao. "Adaptive rejection of stochastic and deterministic sinusoidal disturbances with unknown frequency,". In 2009 American Control Conference, pages 5641-5646, 2009.

[48] D. Huang Y. Xu and L. Zhang. "On-line adaptive identification and cancellation of periodic disturbance with unknown paramete,". In 2009 International Conference on Mechatronics and Automation, pages 2072-2076, 82009.

[49] Q. Zhang. "Periodic disturbance cancellation with uncertain frequency,", 2001.

[50] Q. Zhang and L. J. Brown. "Designing of adaptive bandpass filter with adjustable notch for frequency demodulation,". In American Control Conference, 2003. Proceedings of the 2003, vol. 4, pages 2931-2936, 2003.

[51] Q. Zhang and L. J. Brown. "Noise analysis of an algorithm for uncertain frequency identification,". In IEEE Transactions on Automatic Control, vol. 51, no. 1, pages 103-110, 2006.

[52] Z. Zhao and L. J. Brown. "Musical pitch tracking using internal model control based frequency cancellation,". In 42nd IEEE International Conference on Decision and Control (IEEE, vol. 5, no.03CH37475, pages 5544-5548. Institute of Electrical and Electronics Engineers (IEEE), 122003.

[53] Z. Zhao and L. J. Brown. "Fast estimation of power system frequency using adaptive internalmodel control technique,". In 2004 43rd IEEE Conference on Decision and Control (CDC) (IEEE Cat. No.04CH37601, vol. 1, pages 845-850, 122004. 


\section{Appendix A}

\section{Frequency identification(continue time)}

\section{A.1 Off-Line tuning}

According to what we had discussed earlier in chapter three, where $x_{2 i j}(s)$ is the internal model output that is matching the disturbance $d(t)$.

The responses at $x_{1 i j}(t)$ and $x_{2 i j}(t)$ in steady state are:

$$
\begin{gathered}
x_{1 i j}(t)=\left|x_{i j}\right| \sin \left(j \omega_{i} t+\phi_{i j}\right) \\
x_{2 i j}(t)=\left|x_{i j}\right| \cos \left(j \omega_{i} t+\phi_{i j}\right) \\
x_{i j}=\sqrt{K_{1 i j}^{2} x_{1 i j}^{2}(t)+K_{2 i j}^{2} x_{2 i j}^{2}(t)} \\
\phi_{i j}=\arctan \left(\frac{x_{2 i 1}(t)}{x_{1 i 1}(t)}\right)-j \omega_{i}(t)
\end{gathered}
$$

So, the difference $\Delta \omega_{i}$ between fundamental $\omega_{i}$ and estimated $j \hat{\omega}_{i}$ frequency can be presented as a non-linear function

$$
\Delta \omega=\omega_{i}-\hat{\omega}_{i} \approx \frac{e x_{1 i 1}}{x_{1 i 1}^{2}+x_{2 i 1}^{2}}
$$

A applying simple integrator $K_{a}$ to reduce the difference $\Delta \omega$ and increase the speed of conver- 
gence, so we can rewrite (A.4) as follows

$$
\frac{d w}{d t}=K_{a} \Delta \omega \approx-K_{a} \frac{e x_{1 i 1}}{x_{1 i 1}^{2}+x_{2 i 1}^{2}}
$$

Let a 2nd order desirable bandpas filter be given by

$$
T_{b p}(s)=\frac{d_{1} s^{2}}{s^{4}+c_{1} s^{3}+c_{2} s^{2}+c_{3} s+c_{4}}
$$

The controller parameters are chosen to be such that the transfer function from $d$ to $e$ as follows

$$
\begin{gathered}
T_{b p n}=\frac{d_{1} s^{2}}{s^{4}+c_{1} s^{3}+c_{2} s^{2}+c_{3} s+c_{4}} \times \\
\prod_{i=1}^{n} \frac{s^{2}+\left(j \hat{\omega}_{i}\right)^{2}}{s^{2}+2 \epsilon_{i j} j \hat{\omega}_{i} s+\left(j \hat{\omega}_{i}\right)^{2}}
\end{gathered}
$$

where $\epsilon_{i j}$ are small real numbers, and $j \hat{\omega}_{i}$ are the notches frequency.

$$
\begin{aligned}
T_{d e} & =\frac{G(s)}{1+G(s) \sum_{i=1}^{n} \sum_{j=1}^{m_{i}}\left(\frac{s}{s^{2}+\hat{\omega}_{i}}\right)} \\
& =\frac{b_{1} s^{2} \prod_{i=1}^{n}\left(s^{2}+\left(j \hat{\omega}_{i}\right)^{2}\right.}{a(s) \prod_{i=1}^{n}\left(s^{2}+\left(j \hat{\omega}_{i}\right)^{2}\right)+b_{1} s^{2} \sum s}
\end{aligned}
$$

and

$$
G(s)=\frac{b_{1} s^{2}}{s^{4}+a_{1} s^{3}+a_{2} s^{2}+a_{3} s+a_{4}}=\frac{b_{1} s^{2}}{a(s)} .
$$

\section{A.2 On-line tuning}

A second order Chebyshev band-pass filter was designed with a stopband attenuation of $1 \mathrm{~dB}$, and adjustable low and high band-pass frequency are settled to be $(1 \mathrm{~Hz})$ and $(30 \mathrm{~Hz})$ respectively. Internal models are incorporated. These internal models drive a desired transfer function $T_{d e}$ to be zero at certain frequency; consequently, a band-pass filter and desired band-pass filter with notches are given as follows 


$$
T_{b p}(s)=\frac{3.26210^{04} s^{2}}{s^{4}+200 s^{3}+3.89710^{04} s^{2}+2.36910^{05} s+1.40310^{06}}
$$

and the desired band-pass notches filter is represented as

$$
T_{b p n}=\frac{d_{1} s^{2}}{s^{4}+c_{1} s^{3}+c_{2} s^{2}+c_{3} s+c_{4}} \prod_{i=1}^{n} \prod_{j=1}^{m_{i}} \frac{s^{2}+\left(j \hat{\omega}_{i}\right)^{2}}{s^{2}+2 \epsilon_{i j} j \hat{\omega}_{i} s+\left(j \hat{\omega}_{i}\right)^{2}}
$$

Where, $\epsilon_{i j}$ are small real number, and $j \hat{\omega}_{i}$ is the notches frequency.Therefore, the ability of the algorithm to improve noise cancellation is achieved.

Now, from state spaces form of internal model, it is easy to obtain our transfer function of each internal model $I M_{i j}$, which is given as follows

$$
c(s I-A)^{-1} B
$$

and since the stat space equation for internal model $I M_{i j}$ is given as follows

$$
\begin{gathered}
A_{i j}=\left[\begin{array}{cc}
0 & -j \hat{\omega}_{i} \\
j \hat{\omega}_{i} & 0
\end{array}\right] \quad B_{i j=}\left[\begin{array}{l}
K_{1 i j} \\
K_{2 i j}
\end{array}\right] \\
u_{i j}=\left[\begin{array}{ll}
0 & 1
\end{array}\right] X_{i j}
\end{gathered}
$$

where

$$
\begin{gathered}
X_{i j}=\left[\begin{array}{ll}
x_{1 i j} & x_{2 i j}
\end{array}\right]^{T} \\
=\left[\begin{array}{ll}
0 & 1
\end{array}\right]\left[\begin{array}{cc}
s & j \hat{\omega}_{i} \\
-j \hat{\omega}_{i} & s
\end{array}\right]^{-1}\left[\begin{array}{l}
K_{1 i j} \\
K_{2 i j}
\end{array}\right] \\
=\left[\begin{array}{ll}
0 & 1
\end{array}\right]\left[\begin{array}{cc}
s & -j \hat{\omega}_{i} \\
j \hat{\omega}_{i} & s
\end{array}\right] \frac{1}{s^{2}+\left(j \hat{\omega}_{i}\right)^{2}}\left[\begin{array}{l}
K_{1 i j} \\
K_{2 i j}
\end{array}\right] \Rightarrow \\
=\frac{1}{s^{2}+\left(j \hat{\omega}_{i}\right)^{2}}\left[\begin{array}{cc}
j \hat{\omega}_{i} & s
\end{array}\right]\left[\begin{array}{l}
K_{1 i j} \\
K_{2 i j}
\end{array}\right] \Rightarrow \\
=\frac{1}{s^{2}+\left(j \hat{\omega}_{i}\right)^{2}}\left[\begin{array}{ll}
K_{1 i j} j \hat{\omega}_{i}+K_{2 i j} s
\end{array}\right]
\end{gathered}
$$




$$
I M_{i j}=\frac{K_{2 i j} s+K_{1 i j} j \hat{\omega}_{i}}{s^{2}+\left(j \hat{\omega}_{i}\right)^{2}}
$$

where the tuning transfer function has the following form:

$$
G(s)=\frac{b_{1}(s)}{a(s)}=\frac{b_{1} s^{2}}{s^{4}+a_{1} s^{3}+a_{2} s^{2}+a_{3} s+a_{4}}
$$

Consequently, the transfer function of the closed-loop feedback system is expressed as

$$
\begin{aligned}
T_{d e} & =\frac{G(s)}{1+G(s) \sum_{i=1}^{n} \sum_{j=1}^{m_{i}}\left(\frac{K_{2 i j} s+K_{1 i j} j \hat{\omega}_{i}}{s^{2}+\left(j \hat{\omega}_{i}\right)^{2}}\right)} \\
& =\frac{b_{1} s^{2} \prod\left(s^{2}+(j \hat{\omega})^{2}\right)}{a(s) \prod\left(s^{2}+\left(j \hat{\omega}_{i}\right)^{2}\right)+b_{1} s^{2} \sum\left(K_{2 k l} s+K_{1 k l} l \hat{\omega}_{k}\right) Y_{k l}(s)}
\end{aligned}
$$

where

$$
\begin{gathered}
Y_{k l}=\prod_{i=1}^{n} \prod_{j \neq l}\left(s^{2}+\left(j \hat{\omega}_{i}\right)^{2}\right) \\
i=k
\end{gathered}
$$

At this point and by matching the coefficient of the numerators and denominators in both equations (A.11) and (A.19), values of the control gains $K_{P}$ for each internal model, $b_{1}$ and $a_{i}$ for the tuning function $G(s)$ are obtained. However, we got a set of $2 n+4$ coupled equations with $2 n+4$ unknowns, which is possible to solve in off-line and/or theoretical but not practicable to solve in real time. Alternatively, and since the desired band-pass filter with notches $T_{b p n}$ and input to error signal transfer function $T_{d e}$ are designed, the parameters can be calculated in by matching the coefficients of nominators and denominators in both equation (A.11) and (A.19). In addition, by substituting $s$ in denominators with $\pm\left(\hat{\omega}_{l} \cdot j\right)$ where $l$ is $(1,2, \ldots, n)$ and $j$ is an imaginary number.Thus, the system have reduced to $n$ sets of two equations with two unknowns. Solving these two linear equations the values of the control feedback gains parameters $K_{P}=\left[K_{111} K_{211}, \ldots, K_{1 n m_{n}} K_{2 n m_{n}}\right]$ for each internal model $I M_{i, j}$ can be obtained, where $n$ is number of different frequencies. Moreover, by setting $s=\hat{\omega}_{i}$ in the denominators, which is different values from that are applied to got control gain $K_{P}$, 
we get a set of four equations with four unknowns. Solving these four linear equations, a unique solution for $b_{1}$ and $a_{i}$ can be obtained for the tuning function $G(s)$, where $i=(1,2, \ldots, 4)$.

Since the fundamental frequency $\omega_{i}$ is varying in time, the instantaneous frequency as presented by Hilbert Huang transform can be calculated as

$$
\begin{aligned}
\omega_{i} & =\frac{d}{d t} \angle\left(x_{2 i 1}+j x_{1 i 1}\right) \\
\omega_{i} & =\hat{\omega}_{i}+\frac{e\left(K_{2 i 1} x_{1 i 1}-K_{1 i 1} x_{2 i 1}\right)}{x_{1 i 1}^{2}+x_{2 i 1}^{2}}
\end{aligned}
$$

The equation (A.20) is obtained by substituting equation (3.9) into definition of the derivative of the angel operator. Which is used to update the model and eliminating the error between nominal and the fundamental frequency $\Delta \omega_{i}$ by applying integral feedback control as follows

$$
\dot{\hat{\omega}}_{i}=K_{a} \frac{e\left(K_{2 i 1} x_{1 i 1}-K_{1 i 1} x_{2 i 1}\right)}{x_{1 i 1}^{2}+x_{2 i 1}^{2}}
$$

Therefore, the acceptable level of over shoot and fast convergence are obtained by adjusting integral gain $K_{a}$. 


\section{Appendix B}

\section{Discrete Time}

We did the same procedure here that is already has done in the continues time. Thus, each internal $\left(I M_{i j}\right)$ model in the system can be represented in discrete state space model as follows

$$
\dot{X}_{i j}(T+1)=A_{d i j} X_{i j}(T)+\left[\begin{array}{l}
0 \\
1
\end{array}\right] e(T)
$$

where $i=1,2, \ldots, n$, and $j=1,2, \ldots, m_{i} . A_{d i j}$ is presented by

$$
\begin{gathered}
A_{d i j}=\left[\begin{array}{cc}
\cos \left(\hat{\omega}_{i}\right) & \sin \left(\hat{\omega}_{i}\right) \\
-\sin \left(\hat{\omega}_{i}\right) & \cos \left(\hat{\omega}_{i}\right)
\end{array}\right] \\
u_{i j}(T)=\left[K_{1 i j} K_{2 i j}\right] X_{i j}(T) \\
X_{i j}(T)=\left[x_{1 i j}(T) x_{2 i j}(T)\right]^{T}
\end{gathered}
$$

and adaptive frequency and the magnitude can be calculated as follows

$$
\begin{gathered}
M_{d i j}(T)=\sqrt{x_{1 i}^{2}(T)+x_{2 i}^{2}(T)} \\
\Delta \omega_{i}(T)=\hat{\omega}_{i}(T+1)-\hat{\omega}_{i}(T)=-K_{a} \frac{e x_{1 i j}(T)}{x_{1 i j}^{2}(T)+x_{2 i j}^{2}(T)}
\end{gathered}
$$

Since the $K_{1 i j}$ and $K_{2 i j}$ are chosen to be 0 and 1 respectively, the transfer function for each internal model $\left(I M_{i j}\right)$ from error $e(T)$ to the variable state spaces $x_{2 i j}(T)$ in the discrete form can 
be expressed by

$$
\begin{aligned}
& =C\left(z I-A_{d i j}\right)^{-1} B_{d i j}=\left[\begin{array}{ll}
0 & 1
\end{array}\right]\left[\begin{array}{cc}
z-\cos j \hat{\omega}_{i} & -\sin j \hat{\omega}_{i} \\
\sin j \hat{\omega}_{i} & z-\cos j \hat{\omega}_{i}
\end{array}\right]^{-1}\left[\begin{array}{l}
0 \\
1
\end{array}\right] \\
& =\left[\begin{array}{ll}
0 & 1
\end{array}\right] \frac{1}{\left(z-\cos j \hat{\omega}_{i}\right)^{2}+\sin ^{2} j \hat{\omega}_{i}}\left[\begin{array}{cc}
z-\cos j \hat{\omega}_{i} & \sin j \hat{\omega}_{i} \\
-\sin \omega_{i} & z-\cos j \hat{\omega}_{i}
\end{array}\right]\left[\begin{array}{l}
0 \\
1
\end{array}\right] \\
& =\frac{1}{\left(Z-\cos j \hat{\omega}_{i}\right)^{2}+\sin ^{2} j \hat{\omega}_{i}}\left[\begin{array}{ll}
-\sin j \hat{\omega}_{i} & z-\cos j \hat{\omega}_{i}
\end{array}\right]\left[\begin{array}{l}
0 \\
1
\end{array}\right]
\end{aligned}
$$

so, the transfer function of an $I M_{i j}$ is given by

$$
\begin{array}{r}
\frac{z-\cos \left(j \hat{\omega}_{i}\right)}{\left(z-\cos \left(j \hat{\omega}_{i}\right)\right)^{2}+\sin ^{2}\left(j \hat{\omega}_{i}\right)} \\
\text { or } \frac{z-\cos \left(j \hat{\omega}_{i}\right)}{z^{2}-2 z \cos \left(j \hat{\omega}_{i}\right)+1}
\end{array}
$$

Alternatively, and since $A_{i j}$ in continues time is given by

$$
A_{i j}=\left[\begin{array}{cc}
0 & -j \hat{\omega}_{i} \\
j \hat{\omega}_{i} & 0
\end{array}\right]
$$

SO

$$
\begin{aligned}
\exp ^{A T} & =L^{-1}(s I-A)^{-1}=L^{-1}\left[\begin{array}{cc}
s & j \hat{\omega}_{i} \\
-j \hat{\omega}_{i} & s
\end{array}\right]^{-1} \rightarrow \\
& =L^{-1}\left(\left(\frac{1}{s^{2}+\left(j \hat{\omega}_{i}\right)^{2}}\right)\left[\begin{array}{cc}
s & -j \hat{\omega}_{i} \\
j \hat{\omega}_{i} & s
\end{array}\right]\right)
\end{aligned}
$$

thus,

$$
\begin{aligned}
& =L^{-1}\left[\begin{array}{lc}
\frac{s}{s^{2}+\left(j \hat{\omega}_{i}\right)^{2}} & -\frac{j \hat{\omega}_{i}}{s^{2}+\left(j \omega_{i}\right)^{2}} \\
\frac{j \omega_{i}}{s^{2}+\left(j \hat{\omega}_{i}\right)^{2}} & \frac{s}{s^{2}+\left(j \hat{\omega}_{i}\right)^{2}}
\end{array}\right] \\
A_{d i j} & =\exp ^{A T}=\left[\begin{array}{lc}
\cos \left(j \hat{\omega}_{i}\right) & -\sin \left(j \hat{\omega}_{i}\right) \\
\sin \left(j \hat{\omega}_{i}\right) & \cos \left(j \hat{\omega}_{i}\right)
\end{array}\right]
\end{aligned}
$$

where 


$$
\begin{aligned}
B_{d i j} & =\int_{0}^{T} \exp ^{A T} B \cdot d t \\
& =\int_{0}^{T}\left(\left[\begin{array}{cc}
\cos \left(j \hat{\omega}_{i}\right) & -\sin \left(j \hat{\omega}_{i}\right) \\
\sin \left(j \hat{\omega}_{i}\right) & \cos \left(j \hat{\omega}_{i}\right)
\end{array}\right]\left[\begin{array}{c}
K_{1 i j} \\
K_{2 i j}
\end{array}\right]\right) d t \\
B_{d i j} & =\left[\begin{array}{cc}
\frac{\sin j \hat{\omega}_{i}}{j \hat{\omega}_{i}} & \frac{\cos j \hat{\omega}_{i}}{j \hat{\omega}_{i}} \\
-\frac{\cos j \hat{\omega}_{i}}{j \hat{\omega}_{i}} & \frac{\sin j \hat{\omega}_{i}}{j \hat{\omega}_{i}}
\end{array}\right]
\end{aligned}
$$

The transfer function for each internal model $I M_{i j}$ from error $e(T)$ to the variable state spaces $x_{2 i j}(T)$ in the discrete form can be expressed where $u_{i j}(T)=\left[\begin{array}{ll}0 & 1\end{array}\right] X_{i j}(T)$ and $B_{d i j}=\left[\begin{array}{ll}\bar{K}_{1 i j} & \bar{K}_{2 i j}\end{array}\right]^{T}$ or

$$
B_{d i j}=\left[\begin{array}{c}
\bar{K}_{1 i j} \\
\bar{K}_{2 i j}
\end{array}\right]=\left[\begin{array}{l}
\frac{K_{1 i j} \sin j \hat{\omega}_{i}}{j \hat{\omega}_{i}}+\frac{K_{2 i j} \cos j \hat{\omega}_{i}}{j \hat{\omega}_{i}} \\
\frac{-K_{1 i j} \cos j \hat{\omega}_{i}}{j \hat{\omega}_{i}}+\frac{K_{2 i j} \sin j \hat{\omega}_{i}}{j \hat{\omega}_{i}}
\end{array}\right]
$$

From (B.16) control gains can be calculated as

$$
j \hat{\omega}_{i} \bar{K}_{1 i j}=K_{1 i j} \sin j \hat{\omega}_{i}+K_{2 i j} \cos j \hat{\omega}_{i}
$$

so from (B.17)

$$
\begin{aligned}
K_{1 i j} & =\frac{\bar{K}_{1 i j} j \hat{\omega}_{i}-K_{2 i j} \cos j \hat{\omega}_{i}}{\sin j \hat{\omega}_{i}} \\
j \hat{\omega}_{i} \bar{K}_{2 i j} & =-K_{1 i j} \cos j \hat{\omega}_{i}+K_{2 i j} \sin j \hat{\omega}_{i}
\end{aligned}
$$

by substituting (B.18) into (B.19) so $K_{2 i j}$ can be calculated as follows

$$
\begin{aligned}
j \hat{\omega}_{i} \bar{K}_{2 i j} & =-\left(\frac{\bar{K}_{1 i j} j \hat{\omega}_{i}-K_{2 i j} \cos j \hat{\omega}_{i}}{\sin j \hat{\omega}_{i}}\right) \cdot \cos j \hat{\omega}_{i}+K_{2 i j} \sin j \hat{\omega}_{i} \downarrow \\
j \hat{\omega}_{i} \bar{K}_{2 i j} \sin j \hat{\omega}_{i} & =-\bar{K}_{1 i j} j \hat{\omega}_{i} \cos j \hat{\omega}_{i}+K_{2 i j}\left(\cos j \hat{\omega}_{i}\right)^{2}+K_{2 i j}\left(\sin j \hat{\omega}_{i}\right)^{2} \\
j \hat{\omega}_{i} \bar{K}_{2 i j} \sin j \hat{\omega}_{i}+\bar{K}_{1 i j} j \hat{\omega}_{i} \cos j \hat{\omega}_{i} & =K_{2 i j}\left(\cos ^{2} j \hat{\omega}_{i}+\sin ^{2} j \hat{\omega}_{i}\right) \Rightarrow \\
K_{2 i j} & =j \hat{\omega}_{i} \bar{K}_{2 i j} \sin j \hat{\omega}_{i}+\bar{K}_{1 i j} j \hat{\omega}_{i} \cos j \hat{\omega}_{i}
\end{aligned}
$$

Now we can substitute (B.20) in (B.17), so $K_{1 i j}$ can be calculated as given bellow 


$$
\begin{aligned}
j \hat{\omega}_{i} \bar{K}_{1 i j} & =K_{1 i j} \sin j \hat{\omega}_{i}+\left(j \hat{\omega}_{i} \bar{K}_{2 i j} \sin j \hat{\omega}_{i}+\bar{K}_{1 i j} j \hat{\omega}_{i} \cos j \hat{\omega}_{i}\right) \cdot \cos j \hat{\omega}_{i} \\
j \hat{\omega}_{i} \bar{K}_{1 i j} & =K_{1 i j} \sin j \hat{\omega}_{i}+j \hat{\omega}_{i} \bar{K}_{2 i j} \sin j \hat{\omega}_{i} \cos j \hat{\omega}_{i}+\bar{K}_{1 i j} j \hat{\omega}_{i} \cos ^{2} j \hat{\omega}_{i} \\
K_{1 i j} & =\frac{j \hat{\omega}_{i} \bar{K}_{1 i j}}{\sin j \hat{\omega}_{i}}-\left(\frac{j \hat{\omega}_{i} \bar{K}_{2 i j} \sin j \hat{\omega}_{i} \cos j \hat{\omega}_{i}+\bar{K}_{1 i j} j \hat{\omega}_{i} \cos ^{2} j \hat{\omega}_{i}}{\sin j \hat{\omega}_{i}}\right) \Rightarrow \\
K_{1 i j} & =\frac{j \hat{\omega}_{i} \bar{K}_{1 i j}}{\sin j \hat{\omega}_{i}}-\cot j \hat{\omega}_{i}\left(j \hat{\omega}_{i} \bar{K}_{2 i j} \sin j \hat{\omega}_{i}+\bar{K}_{1 i j} j \hat{\omega}_{i} \cos j \hat{\omega}_{i}\right)
\end{aligned}
$$

The transfer function of an $I M_{i j}$ when $G(s) \neq 1$ is given by

$$
\begin{aligned}
& =C\left(z I-A_{d i j}\right)^{-1} B_{d i j} \\
& =\left[\begin{array}{ll}
0 & 1
\end{array}\right]\left[\begin{array}{cc}
z-\cos j \hat{\omega}_{i} & \sin j \hat{\omega}_{i} \\
-\sin j \hat{\omega}_{i} & z-\cos j \hat{\omega}_{i}
\end{array}\right]^{-1}\left[\begin{array}{c}
\bar{K}_{1 i j} \\
\bar{K}_{2 i j}
\end{array}\right] \\
& =\left[\begin{array}{ll}
0 & 1
\end{array}\right] \frac{1}{\left(z-\cos j \hat{\omega}_{i}\right)^{2}+\sin ^{2} j \hat{\omega}_{i}}\left[\begin{array}{cc}
z-\cos j \hat{\omega}_{i} & -\sin j \hat{\omega}_{i} \\
\sin \omega_{i} & z-\cos j \hat{\omega}_{i}
\end{array}\right]\left[\begin{array}{l}
\bar{K}_{1 i j} \\
\bar{K}_{2 i j}
\end{array}\right] \\
& =\frac{1}{\left(Z-\cos j \hat{\omega}_{i}\right)^{2}+\sin ^{2} j \hat{\omega}_{i}}\left[\sin j \hat{\omega}_{i} \quad z-\cos j \hat{\omega}_{i}\right]\left[\begin{array}{l}
\bar{K}_{1 i j} \\
\bar{K}_{2 i j}
\end{array}\right] \\
& \rightarrow=\frac{\bar{K}_{1 i j} \sin j \hat{\omega}_{i}+\bar{K}_{2 i j}\left(z-\cos j \hat{\omega}_{i}\right)}{\left(Z-\cos j \hat{\omega}_{i}\right)^{2}+\sin ^{2} j \hat{\omega}_{i}} \downarrow \\
& \frac{\bar{K}_{2 i j} z-\bar{K}_{2 i j} \cos \left(j \hat{\omega}_{i}\right)+\bar{K}_{1 i j} \sin \left(j \hat{\omega}_{i}\right)}{z^{2}-2 z \cos \left(j \hat{\omega}_{i}\right)+1} .
\end{aligned}
$$

and

$$
\begin{aligned}
& \bar{K}_{2 i j}=\overline{\bar{K}}_{2 i j} \\
& \bar{K}_{1 i j}=\frac{\overline{\bar{K}}_{1 i j}+\overline{\bar{K}}_{2 i j} \cos j \hat{\omega}_{i}}{\sin j \hat{\omega}_{i}}
\end{aligned}
$$

A Chebyshev second order band-pass filter is incorporated in with a stopband attenuation of $1 \mathrm{~dB}$, and low $\omega_{L}$ and high $\omega_{H}$ band-pass frequency is settled to $1 \mathrm{~Hz}$ and $30 \mathrm{~Hz}$ respectively

$$
T_{b p}(z)=\frac{n_{1} z^{4}+n_{2} z^{3}+n_{3} z^{2}+n_{4} z+n_{5}}{z^{4}+d_{1} z^{3}+d_{2} z^{2}+d_{3} z+d_{4}}
$$

Therefore,band-pass notches filter and the transfer function of the closed loop feedback system 
are represented respectively by

$$
\begin{gathered}
T_{b p n}(z)=\frac{n_{1} z^{4}+n_{2} z^{3}+n_{3} z^{2}+n_{4} z+n_{5}}{z^{4}+d_{1} z^{3}+d_{2} z^{2}+d_{3} z+d_{4}} \times \\
\prod_{i=1}^{n} \prod_{l=1}^{m_{i}} \frac{\left(z-e^{l \hat{\omega}_{k} \sqrt{-1}}\right)\left(z-e^{-l \hat{\omega}_{k} \sqrt{-1}}\right)}{\left(z-e^{-l \hat{\omega}_{k} \varepsilon_{k l}+j \hat{\omega}_{k} l} \sqrt{1-\epsilon_{k l}^{2}}\right)\left(z-e^{-l \hat{\omega}_{k} \epsilon_{k l}-j \hat{\omega}_{k} l} \sqrt{1-\epsilon_{k l}^{2}}\right)} \\
T_{d e}(z)=\frac{G(z)}{1+G(z) \sum_{i=1}^{n} \sum_{j=1}^{m_{i}}\left(\frac{\bar{K}_{2 i j} z-\bar{K}_{1 i j} \sin j \hat{\omega}_{i}+\bar{K}_{2 i j} \cos j \hat{\omega}_{i}}{z^{2}-2 \cos j \hat{\omega}_{i} z+1}\right)} \\
b(z) \prod_{i=1}^{n} \prod_{j=1}^{m_{i}}\left(z^{2}-2 \cos j \hat{\omega}_{i} z+1\right) \\
a(z) \prod\left(z^{2}-2 \cos j \hat{\omega}_{i} z+1\right)+b(z) \sum_{k=1}^{n} \sum_{l=1}^{m_{k}}\left(\bar{K}_{2 k l} z-\bar{K}_{1 k l} \sin j \hat{\omega}_{i}+\bar{K}_{2 k l} \cos j \hat{\omega}_{i}\right) Y_{k l}(z)
\end{gathered}
$$

where

$$
\begin{aligned}
Y_{k l}=\prod_{i=1}^{n} \prod_{j \neq l}\left(z^{2}-2 \cos j \hat{\omega}_{i} z+1\right) \\
i=k
\end{aligned}
$$

The terms $Y_{k l}$ are the product of all the terms $\left(z^{2}-2 \cos j \hat{\omega}_{i} z+1\right)$ except the $i=k, j=l$ term where the tuning function is expressed in the form of

$$
G(z)=\frac{M(z)}{N(z)}=\frac{b_{1} z^{4}+b_{2} z^{3}+b_{3} z^{2}+b_{4} z+b_{5}}{z^{4}+a_{1} z^{3}+a_{2} z^{2}+a_{3} z+a_{4}}=\frac{b(z)}{a(z)}
$$

In order to get a unique solution for controller parameters $b(z)$ and $a(z)$ for $G(z)$ and the feedback gains $K_{d}=\left\{K_{111} K_{211}, \ldots, K_{1 n m_{n}} K_{2 n m_{n}}\right\}$ for each $I M_{i j}$, we need to match the coefficients in both equation (B.29) and (B.30).

Therefore, $K_{1 k l}$ and $K_{2 k l}$ can be obtained by substituting $z=e^{ \pm l \hat{\omega}_{i} \sqrt{-1}}$ in the denominators, where $i$ is $(1,2 \ldots, n)$ and $j$ is imaginary number. Furthermore, setting $z$ to the different values from that have chosen to calculate $K_{d}$, we generate four liner equation in $a_{i}(z)$, we solve these four equation, $a_{1}, a_{2}, a_{3}$ and $a_{4}$ values can be obtained. 


\section{Appendix C}

\section{Proposed algorithm Matlab code}

The mat-lab code contain two main parts .First is (IFD), following this code indicates the design of Chebyshev second order band-pass filter $T_{b p}$, desired band-pass filter with notches $T_{b p n}$, tuning function $G(s)$ and closed loop transfer function $T_{d e}$. As a result, the state space variables gain $K_{p}$ for each $I M_{i, j}$ and the parameters $b_{1}, a_{1}, a_{2}, a_{3}$, and $a_{4}$ for the tuning function are computed based on the desired estimation system behaves such as a band-pass filter with notches.Second part is the S-function which is indicate the states variables derivation $\dot{x}_{1 i j}$ and $\dot{x}_{2 i j}$ for the system, initial conditions for the states variables and estimated frequencies $j \hat{\omega}_{1}$ and $j \hat{\omega}_{2}$ for each internal model $I M_{i, j}$. As well as the number of the inputs $m$, adaptive gain $K_{a}$ and number of notches $n$.

\section{C.1 IFD}

$/ /$ Function $[\mathrm{kp}, \mathrm{a}, \mathrm{b}]=\operatorname{parameters}(\omega, E)$

//Design Chebyshev second order band-pass filter $T_{b p}$

$$
\begin{aligned}
& \omega_{H}=30 * 2 * \mathrm{pi} ; \\
& \omega_{L}=1 * 2 * \mathrm{pi} ; \\
& {\left[\mathrm{ch}_{b}, \mathrm{ch}_{a}\right] \operatorname{cheby} 1\left(2,1\left[\begin{array}{ll}
\omega_{H} & \omega_{L}
\end{array}\right],{ }^{\prime}{ }^{\prime}\right) ;} \\
& \mathrm{c} 1=\mathrm{ch} \_\mathrm{a}(2) ; \\
& \mathrm{c} 2=\text { ch_a(3); }
\end{aligned}
$$




$$
\begin{aligned}
& \mathrm{c} 3=\mathrm{ch} \_\mathrm{a}(4) ; \\
& \mathrm{c} 4=\mathrm{ch} \_\mathrm{a}(5) ; \\
& \mathrm{b}=\mathrm{ch} \_\mathrm{b}(3) ;
\end{aligned}
$$

$/ /$ Read the input $(\omega, E)$

//Find the identical frequencies

//Identify the variables

// For counter $i$ is equal to $1:$ floor(M/2)

// Find equal or adjacent frequencies

$$
\operatorname{ind} x=\operatorname{find}(\omega(\mathrm{i}+1 \text { :end })>(\omega(\mathrm{i}) *(1-.005)) \& \omega(\mathrm{i}+1 \text { :end })<(\omega(\mathrm{i}) *(1+.005))) \text {; }
$$

$$
\text { // IF true }
$$

// Update the frequencies

// increment the counter by one

// Drop the second matched one and save it in a new variable

$$
\text { Dro }=\text { ind } x(1)+i+\text { counter }-1 \text {; }
$$

$\operatorname{drop}($ counter $)=$ Dro;

// Decrement the length of input by one

$$
\mathrm{M}=\mathrm{M}-1
$$

// Save the first matched frequency in new variable (kept)

$$
\begin{aligned}
& \operatorname{kept}(\text { counter })=\mathrm{i} ; \\
& / / \text { END IF } \\
& / / \text { END FOR } \\
& \mathrm{wt}=\mathrm{w}(1: \mathrm{M}) ; \\
& \text { para_de }=\text { ones }(1, \mathrm{M}) ; \\
& \text { den_bp0 }=[1 \mathrm{c} 1 \mathrm{c} 2 \mathrm{c} 3 \mathrm{c} 4] \\
& \text { den_bpn_3 = } \omega t^{2}
\end{aligned}
$$

FOR $\mathrm{k}=1: \mathrm{M}$

//Return the value of a polynomial lowpass filter denominator at $(j * \omega)$ 
den_bpn0_1 = polyval $($ den_bp $0, j * \omega(k)) ;$

//Substitute the first term in the notch filter denominator by $(j * \omega)$

den_bpn_1 $=(j * \omega(k))^{2}$

// Substitute the value of the second term of the notch filter denominator by $(j * \omega)$

den_bpn_2 $=2 * \mathrm{E}(1: \mathrm{M}) . * \omega t^{*}(j * \omega(k)) ;$

//Add notches filter terms

den_bpn_1 = den_bpn_1 + den_bpn_2 +den_bpn_3;

// Computing the lowpass filter with notches by multiplying by the lowpass denominator with the product of notches filter

den_bpn_1_1= den_bpn0_1*prod(den_bpn_1);

// Calculating the feedback closed loop transfer function $T_{d e}$

para_de $=\left(\omega t-\left(\omega(k)^{2}\right)\right.$;

para_de $($ para_de $==0)=1$;

para_de_1 $=\mathrm{b} *\left(j * \omega(k)^{2}\right) * \operatorname{prod}($ para_de $) ;$

// Calculate the gains for the internal models

$\mathrm{kp}(2 * \mathrm{k}-1)=\operatorname{real}\left(\mathrm{den} \_b p n \_1 \_1 /(\right.$ para_de_1 $\left.* \omega(k))\right) ;$

$\operatorname{kp}(2 * \mathrm{k})=\operatorname{imag}\left(\mathrm{den} \_\mathrm{bpn} \_1 \_1 /(\right.$ para_de_1* $\left.\omega(k))\right) ;$

\section{END FOR}

IF the counter true then

//Dropping an internal model $I M_{l ; k}$ from the design stage

FOR $i=1$ :counter

$\operatorname{kp}(2 * \operatorname{kept}(i)-1: 2 * \operatorname{kept}(\mathrm{i}))=\operatorname{kp}(2 * \operatorname{kept}(\mathrm{i})-1: 2 * \operatorname{kept}(\mathrm{i})) / 2$;

$\operatorname{kp}(2 * \operatorname{drop}(i)-1: \operatorname{end})=[\operatorname{kp}(2 * \operatorname{kept}(\mathrm{i})-1: 2 * \operatorname{kept}(\mathrm{i})) / 2 \operatorname{kp}(2 * \operatorname{drop}(\mathrm{i})-1: \operatorname{end}-2)]$;

\section{END FOR}

END IF

$/ /$ Computing the $a_{i}$ parameter for the tuning function $G(s)$

w_squa $=\omega 0 .^{2} ;$ 


\section{APPENDIX C. PROPOSED ALGORITHM MATLAB CODE}

$\mathrm{w} \_2 \mathrm{eb}=2 * \mathrm{E} 0 . * \omega 0 ;$

a3_sumprod $=\operatorname{prod}\left(\mathrm{w} \_s q u a\right) *$ w_2eb./w_squa;

a2_4eb=w_2eb(1:9).*cumsum(w_2eb(2:end),'reverse');

$\mathrm{a} 1=\mathrm{c} 1+\operatorname{sum}\left(\mathrm{w} \_2 \mathrm{eb}\right)$;

$\mathrm{a} 2=\operatorname{sum}\left(\mathrm{a} 2 \_4 \mathrm{eb}\right)+\mathrm{c} 1 * \operatorname{sum}\left(\mathrm{w} \_2 \mathrm{eb}\right)+\mathrm{c} 2$;

a3 $=\left(\right.$ c3*prod $($ w_squa $)+\mathrm{c} 4 *$ sum $\left(\mathrm{a} 3 \_\right.$sumprod $\left.)\right) /$prod(w_squa $)$;

$\mathrm{a} 4=\mathrm{c} 4$

$\mathrm{a}=[\mathrm{a} 1 \mathrm{a} 2 \mathrm{a} 3 \mathrm{a} 4]$

\section{END FUNCTION}




\section{C.2 S-Function}

$\% \% \%$ Initialize values $\% \% \%$

//Switch Flag

//Case 0

$/ / \mathrm{IF}$

length(n) =1 \& length(n) =m, error('number of $I M_{i, j}$ inconsistent')

END IF

$\operatorname{str}=[]$

$\mathrm{s}=$ simsizes ;

s.NumContStates $=2 * \operatorname{sum}(\mathrm{n})+\mathrm{m}$;

s.NumDiscStates $=0$;

s.NumOutputs $=2 * \operatorname{sum}(n)+m$;

s.NumInputs $=2 * \operatorname{sum}(\mathrm{n})+1$;

s.DirFeedthrough $=0$;

s.NumSampleTimes $=1$;

sys $=\operatorname{simsizes}(\mathrm{s})$;

$\mathrm{x} 0=\mathrm{IN}$;

//States variables Derivation

//Case 1

$\mathrm{e}=\mathrm{u}(1)$

$\mathrm{n} 1=0$;

$\mathrm{n} 2=2 * \operatorname{sum}(\mathrm{n}) ;$

//FOR i=1:m;

//FOR j=2:2:2*n(i);

$\mathrm{k} 1=\mathrm{u}(\mathrm{n} 1+\mathrm{j})$

$\mathrm{k} 2=\mathrm{u}(\mathrm{n} 1+\mathrm{j}+1) ;$

$\mathrm{dx}(\mathrm{n} 1+\mathrm{j}-1)=-(\mathrm{j} / 2) * \mathrm{x}(\mathrm{n} 2+\mathrm{i}) * \mathrm{x}(\mathrm{n} 1+\mathrm{j})+\mathrm{k} 1 * \mathrm{e} ;$ 
$\mathrm{dx}(\mathrm{n} 1+\mathrm{j})=(\mathrm{j} / 2) * \mathrm{x}(\mathrm{n} 2+\mathrm{i}) * \mathrm{x}(\mathrm{n} 1+\mathrm{j}-1)+\mathrm{k} 2 * \mathrm{e}$

//END FOR

//Applying the adaptive gain into estimated frequency

$/ / \mathrm{IF} \mathrm{t}<.25 \mid \mathrm{x}(\mathrm{n} 1+1)^{2}+\mathrm{x}(\mathrm{n} 1+2)^{2}<.4 ;$

$\mathrm{dx}(\mathrm{n} 2+\mathrm{i})=0 ;$

//ELSE

$\mathrm{dx}(\mathrm{n} 2+\mathrm{i})=\mathrm{Ka}(\mathrm{i}) *(\mathrm{x}(\mathrm{n} 1+1) * \mathrm{u}(\mathrm{n} 1+3)-\mathrm{x}(\mathrm{n} 1+2) * \mathrm{u}(\mathrm{n} 1+2)) * \mathrm{e} /\left(\mathrm{x}(\mathrm{n} 1+1)^{2}+\mathrm{x}(\mathrm{n} 1+2)^{2}\right) ;$

$\mathrm{n} 1=\mathrm{n} 1+2 * \mathrm{n}(\mathrm{i}) ;$

//END IF

//END FOR

sys $=\mathrm{dx}$;

//CASE 3,

sys $=\mathrm{x}$;

//CASE $\{249\}$

sys[];

//Otherwise

error(['Unhandled flag = ',num2str(flag)]);

//END SWITCH CASE 


\section{Appendix D}

\section{Mat-lab alternative approach code}

The mat-lab code here shows the alternative approch which is also contain two main parts as the propsed algoritghm cod (IFD) and the (SF). We have shown how we did replaced the bandpass filter by lowpass filter and modfiid the reast of the code due to that changing. Thus, the code can be given as

\section{D.1 IFD}

Function $[\mathrm{kp}, \mathrm{a}, \mathrm{b}]=\operatorname{parameters}(\omega, E)$

//Design Chebyshev second order lowpass filter

$/ /$ Read the input $(\omega, \epsilon)$

$$
\begin{gathered}
\omega_{H}=30 * 2 * p i \\
\omega_{L}=1 * 2 * p i \\
B W=\left(\omega_{H}-\omega_{L}\right) \\
{\left[c h_{b}, c h_{a}\right]=\text { cheby } 1\left(2,1, B W^{\prime} s^{\prime}\right)} \\
/ / \text { Identify the variables } \\
\text { c1 = ch_a(2) } \\
\text { c2 = ch_a(3) } \\
\text { b }=\text { ch_b(3) }
\end{gathered}
$$

//Identify the variables 


$$
\begin{gathered}
\mathrm{M}=\text { length }(\omega) ; \\
\mathrm{kp}=\operatorname{ones}(1,2 * \mathrm{M}) ; \\
\text { counter=0; } \\
\text { kept=zeros }(1, \mathrm{M}-1) ; \\
\text { drop=zeros }(1, \mathrm{M}-1) ; \\
\text { ind }=\text { zeros; } \\
\text { FOR } \mathrm{i}=1 \text { :floor }(\mathrm{M} / 2)
\end{gathered}
$$

// Find equal or adjacent frequencies

$$
\operatorname{indx}=\operatorname{find}(\omega(\mathrm{i}+1 \text { :end })>(\omega(\mathrm{i}) *(1-.001)) \& \omega(\mathrm{i}+1 \text { :end })<(\omega(\mathrm{i}) *(1+.001))) \text {; }
$$

// IF true

// increment the counter by one

IF length(indx)

$$
\text { counter }=\text { counter }+1 \text {; }
$$

// Drop the second matched one and save it in a new variable

$$
\text { Dro }=\text { ind } x(1)+i+\text { counter }-1 \text {; }
$$$$
\operatorname{drop}(\text { counter })=\text { Dro; }
$$

// Update the frequencies

$$
\omega(1 \text { :end-counter })=\mathrm{w}([1 \text { :ind } x(1)+\mathrm{i}-1 \text { Dro-counter }+2 \text { :end-counter }+1]) \text {; }
$$

// Update the epsilons

$$
\mathrm{E}(1 \text { :end-counter })=\mathrm{E}([1 \text { :ind }(1)+\mathrm{i}-1 \text { Dro-counter }+2 \text { :end-counter }+1]) \text {; }
$$

// Decrement the length of input by one

$$
\mathrm{M}=\mathrm{M}-1
$$

// Save the first matched frequency in new variable

$$
\operatorname{kept}(\text { counter })=\mathrm{i}
$$

END IF

\section{END FOR}

$$
\mathrm{wt}=\mathrm{w}(1: \mathrm{M}) ;
$$


para_de $=$ ones $(1, \mathrm{M})$

den_bp0 = [ 1 c c1 c2 $]$;

den_bpn_3 $=\omega t^{2}$

FOR $\mathrm{k}=1: \mathrm{M}$

//Return the value of a polynomial lowpass filter denominator at $(j * \omega)$

den_bpn0_1 = polyval $($ den_bp0, $j * \omega(k))$;

//Substitute the first term in the notch filter denominator by $(j * \omega)$

den_bpn_1 $=(j * \omega(k))^{2}$

// Substitute the value of the second term of the notch filter denominator by $(j * \omega)$

den_bpn_2 $=2 * \mathrm{E}(1: \mathrm{M}) . * \omega t^{*}(j * \omega(k)) ;$

//Add notches filter terms

den_bpn_1 = den_bpn_1 + den_bpn_2 + den_bpn_3;

// Computing the lowpass filter with notches by multiplying by the lowpass denominator with the product of notches filter

den_bpn_1_1 = den_bpn0_1*prod(den__bpn_1);

// Calculating the feedback closed loop transfer function $T_{d e}$

para_de $=\left((j * \omega(k))^{2}+\omega t^{2}\right) ;$

para_de $($ para_de $==0)=1$;

para_de_1 $\_\mathrm{b} *(j * \omega(k)) * \operatorname{prod}($ para_de $)+\mathrm{b}^{*} \operatorname{prod}\left((j * \omega(k))^{2}+\omega t^{2}\right)$;

$\mathrm{kp}(2 * \mathrm{k}-1)=\operatorname{real}\left(\mathrm{den} \_\mathrm{bpn} \_1 \_1 /\left(\operatorname{para} \_d e \_1 * \omega(k)\right)\right) ;$

$\mathrm{kp}(2 * \mathrm{k})=$ imag $($ den_bpn_1_1/(para_de_1* $\omega(k))) ;$

\section{END FOR}

IF the counter true then

//Dropping an internal model $I M_{l ; k}$ from the design stage

$$
\begin{aligned}
& \text { FOR } i=1 \text { :counter } \\
& \begin{array}{l}
\mathrm{kp}(2 * \operatorname{kept}(\mathrm{i})-1: 2 * \operatorname{kept}(\mathrm{i}))=\operatorname{kp}(2 * \operatorname{kept}(\mathrm{i})-1: 2 * \operatorname{kept}(\mathrm{i})) / 2 ; \\
\mathrm{kp}(2 * \operatorname{drop}(\mathrm{i})-1: \operatorname{end})=[\operatorname{kp}(2 * \operatorname{kept}(\mathrm{i})-1: 2 * \operatorname{kept}(\mathrm{i})) / 2 \mathrm{kp}(2 * \operatorname{drop}(\mathrm{i})-1: \text { end }-2)]
\end{array}
\end{aligned}
$$




\section{END FOR}

\section{END IF}

// Computing the $a$ parameter for the tuning function $G(s)$

$a=c 1+\operatorname{sum}(2 * E 0 . * \omega 0) ;$

\section{END FUNCTION}




\section{D.2 S-Function}

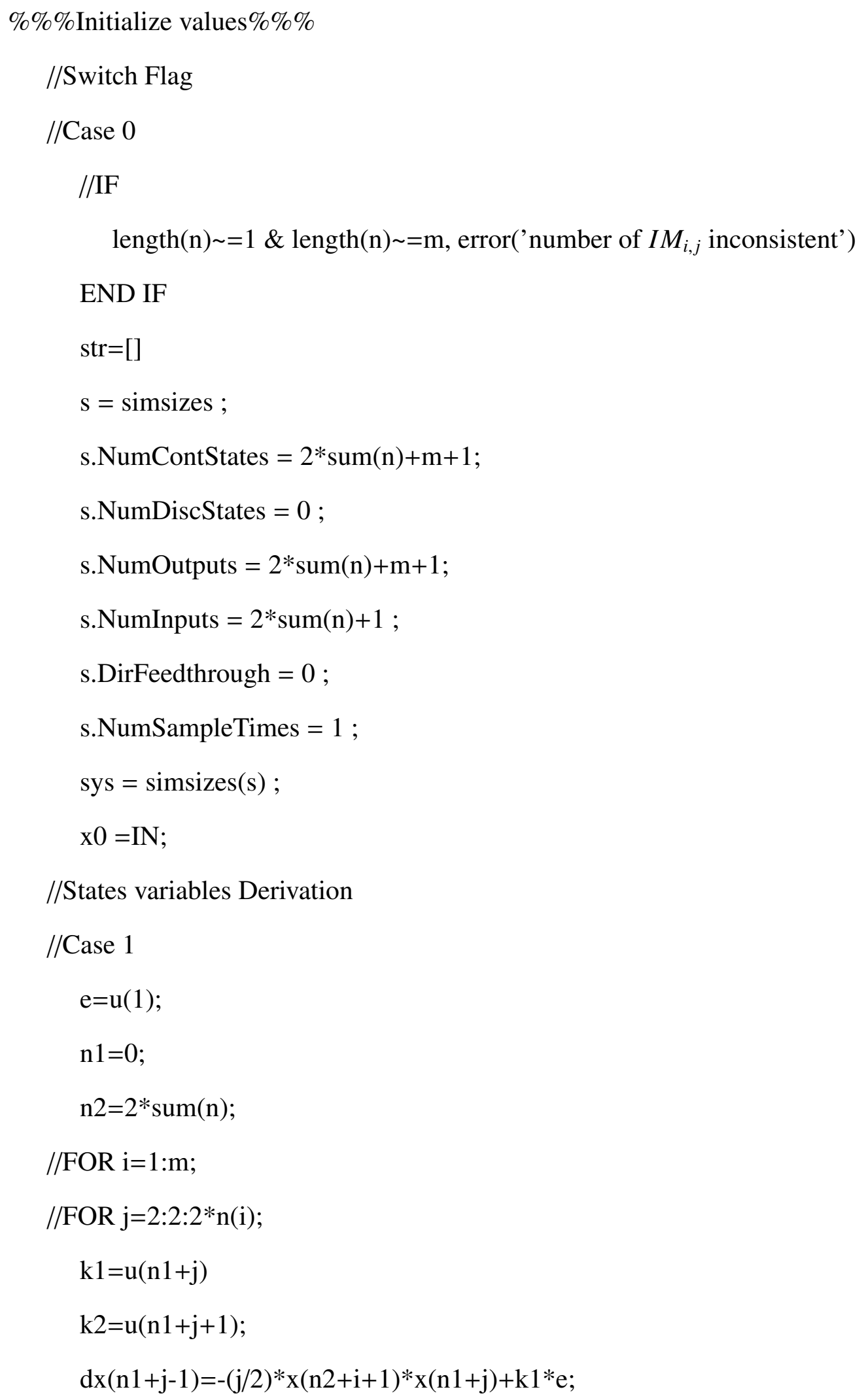


$\mathrm{dx}(\mathrm{n} 1+\mathrm{j})=(\mathrm{j} / 2) * \mathrm{x}(\mathrm{n} 2+\mathrm{i}+1) * \mathrm{x}(\mathrm{n} 1+\mathrm{j}-1)+\mathrm{k} 2 * \mathrm{e}$

//END FOR

//Applying the adaptive gain into estimated frequency

$/ / \mathrm{IF} \mathrm{t}<.25 \mid \mathrm{x}(\mathrm{n} 1+1)^{2}+\mathrm{x}(\mathrm{n} 1+2)^{2}<.4 ;$

$\mathrm{dx}(\mathrm{n} 2+\mathrm{i}+1)=0 ;$

//ELSE

$\mathrm{dx}(\mathrm{n} 2+\mathrm{i}+1)=\mathrm{Ka}(\mathrm{i}) *(\mathrm{x}(\mathrm{n} 1+1) * \mathrm{u}(\mathrm{n} 1+3)-\mathrm{x}(\mathrm{n} 1+2) * \mathrm{u}(\mathrm{n} 1+2))^{*} \mathrm{e} /\left(\mathrm{x}(\mathrm{n} 1+1)^{2}+\mathrm{x}(\mathrm{n} 1+2)^{2}\right) ;$

$\mathrm{n} 1=\mathrm{n} 1+2 * \mathrm{n}(\mathrm{i})$

//END IF

//END FOR

sys $=\mathrm{dx}$;

$\mathrm{dx}(\mathrm{n} 2+1)=\mathrm{e} ;$

//CASE 3,

sys $=x$;

//CASE $\{249\}$

sys $=[]$

//Otherwise

error(['Unhandled flag = ',num2str(flag)]);

//END SWITCH CASE 


\title{
Curriculum Vitae
}

\author{
Name: $\quad$ Edris Mohsen \\ Post-Secondary University of Western Ontario \\ Education and London, ON, Canada \\ Degrees: 2017 Master of Science in Electrical and Computer Engineering \\ College of Electronic Technology \\ Tripoli, Libya \\ 2008 Higher Diploma Degree (Automatic Control) \\ The Libyan Academy \\ Tripoli, Libya \\ 2011 Bachelor in (Electrical Engineering and Computer Science) \\ The Libyan Academy \\ Tripoli, Libya \\ 2013 Master Diploma ( Applied and Engineering Science)
}

Honours and Ministry of Higher Education and Scientific Research Scholarship (MHESR)

Awards:

College of Electronic Technology

2008

Related Work Graduate Teaching and Research Assistant

Experience: $\quad$ University of Western Ontario

2015-2017

Instrument Planning Analyst

Mellitah Oil \& Gas B.V Company

2014-now

Surface system Engineer

Schlumberger Company 
2009-2010

Instrument Technician

Mellitah Oil \& Gas B.V Company

2001-2008

Publications: $\quad$ E. Mohsen and L. J. Brown. Realtime implementation of an internal-modelprinciple signal identifier. In 2017 IEEE 30th Canadian Conference on Electrical and Computer Engineering (CCECE), pages 1-5, April 2017.

E. Mohsen L. J. Brown and J. Chen. A real time alternative to the hilbert huang transform based on internal model principle. J Electr Electron, (Syst 6: 233), July 2017.

E. Mohsen L. J. Brown. Improved design of an adaptive internal model principle signal analyzer. ICASSP 2018 : IEEE International Conference on Acoustics, Speech and Signal Processing, pages 1-4, April 2018.

J. Chen L. J. Brown and E. Mohsen. Signal identification based on internal model in discrete time. ICASSP 2018 : IEEE International Conference on Acoustics, Speech and Signal Processing, pages 1-4, April 2018 